\title{
Labeled Bipolar Argumentation Frameworks
}

\author{
Melisa G. Escañuela Gonzalez \\ Departamento de Matermática \\ Universidad Nacional de Santiago del Estero (UNSE) \\ Consejo Nacional de Investigaciones Científicas y Técnicas (CONICET) \\ Sgo. del Estero, Argentina \\ Maximiliano C. D. Budán \\ Departamento de Matermática \\ Universidad Nacional de Santiago del Estero (UNSE) \\ Consejo Nacional de Investigaciones Científicas y Técnicas (CONICET) \\ Sgo. del Estero, Argentina
}

Gerardo I. Simari

Departamento de Ciencias e Ingeniería de la Computación

Universidad Nacional del Sur (UNS)

Instituto de Ciencias e Ingeniería de la Computación (ICIC UNS-CONICET)

Bahía Blanca, Argentina

\section{Guillermo R. Simari}

Departamento de Ciencias e Ingeniería de la Computación

Universidad Nacional del Sur (UNS)

Instituto de Ciencias e Ingeniería de la Computación (ICIC UNS-CONICET)

Bahía Blanca, Argentina

MELIESCA@UNSE.EDU.AR

MCDB@CS.UNS.EDU.AR

GIS@CS.UNS.EDU.AR

GRS@CS.UNS.EDU.AR

\begin{abstract}
An essential part of argumentation-based reasoning is to identify arguments in favor and against a statement or query, select the acceptable ones, and then determine whether or not the original statement should be accepted. We present here an abstract framework that considers two independent forms of argument interaction-support and conflict—and is able to represent distinctive information associated with these arguments. This information can enable additional actions such as: $(i)$ a more in-depth analysis of the relations between the arguments; (ii) a representation of the user's posture to help in focusing the argumentative process, optimizing the values of attributes associated with certain arguments; and (iii) an enhancement of the semantics taking advantage of the availability of richer information about argument acceptability. Thus, the classical semantic definitions are enhanced by analyzing a set of postulates they satisfy. Finally, a polynomial-time algorithm to perform the labeling process is introduced, in which the argument interactions are considered.
\end{abstract}

\section{Introduction and Motivation}

Argumentation aims towards formalizing reasoning mechanisms with the capability of handling contradictory, incomplete, and/or uncertain information, taking as inspiration commonsense reasoning and the human-like mechanism of defending a statement by giving reasons for its acceptance and analyzing the ones against it (Rahwan \& Simari, 2009). In this process, since reasons supporting conflicting conclusions can also be advanced, both the original statement and its support are subject to scrutiny. Argumentation theories have been proposed for applications in many different 
domains, such as legal reasoning (Prakken \& Sartor, 1997), recommender systems (Chesñevar \& Maguitman, 2004), agents and multi-agent systems (Kraus, 1997), applications to cyber security by Shakarian et al. (2016, 2018), and others (Poole, Mackworth, \& Goebel, 1998; Vreeswijk, 1997).

Several argument-based formalisms have emerged to study the different relations among arguments. Dung (1995) proposes Abstract Argumentation Frameworks (AFs) to model real-world situations representing the attack relations between abstract entities called arguments, and providing different acceptability semantics to determine which sets of arguments are acceptable. Subsequently, Cayrol and Lagasquie-Schiex (2005b) extended Dung's framework taking into account two independent relations between arguments: attack and support. In this formalism, called Bipolar Argumentation Frameworks (BAFs), the authors model a bipolar reasoning mechanism where those arguments in favor of a conclusion can be considered as positive while those against the conclusion as negative. Furthermore, they adapt Dung's acceptability semantics taking into account the support relationship between arguments. Following the introduction of BAFs, several relevant research lines that explored this formalism in different directions have been pursued by Nouioua et al. (2010), Amgoud et al. (2008), Boella et al. (2010), and Cohen et al. (2018).

Although these formalizations model aspects that correspond to real-world situations, they do not provide tools to represent particular features of arguments, and how these features are affected by the relations (support and attack) between them. However, there exist applications where it is necessary to provide such modeling details, considering features in order to refine the analysis and provide extra information about their acceptance (Bench-Capon, 2003). That is, these formalisms cannot provide the tools to represent the features associated with the arguments in relation to the application domain such that: the reliability degrees associated with the information sources in a recommender system, the jurisdiction and/or intuition behind legal arguments in the study of a legal case, the temporal information associated with arguments involved in a decision support system, among others. Thus, our approach allows us to represent how much a given argument is "believed or disbelieved" by a given person analyzing different dimensions, offering us the possibility to express more than just the classical acceptability status.

Considering this issue, Hunter and Polberg (2018) explain that exploratory study shows that the most common approaches to argumentation might be too simplistic in order to adequately grasp human reasoning. However, they do not believe that argumentation theory as a field is insufficient altogether-in particular, they highlight the correspondence between the obtained results and various, less common, formalisms such as probabilistic and bipolar frameworks, and prudent and careful approaches. In this direction, they show how descriptive models of argumentation should take into consideration bipolar approaches and graded/ranking semantics jointly based on the following aspects: (i) Analysis of how many weights the participants were using throughout the dialogs. In most cases, three weights were insufficient to represent the participants' opinions; a more detailed view of their behavior was required to perform an adequate semantic analysis over complex scenarios where classic semantics may fail to explain. (ii) Many additional attacks perceived by the participants can be explained by the existing notions of indirect conflict in the bipolar argumentation framework. They can therefore be used to model auxiliary conflicts arising in the context of a dialogue, but not necessarily created at the logical level. Furthermore, the participants explicitly view certain relations as supporting, and the notion of defense does not account for all of the positive relations that the participants have identified between the presented statements. In particular, they observed that there are new support relations arising in the context of the dialogue, such as support coming from statements working towards the same goal. 
In this work, based on the previous analysis, we have extended $B A F$ by taking into account the properties associated with the arguments in the form of labels, increasing the representational capabilities of this formalization. These labels can be combined and propagated through the bipolar argumentation graph in accordance with the arguments' interaction. Then, considering the additional information provided by these labels, the semantics offered by $B A F$ is improved by: $(i)$ obtaining more information about the arguments, (ii) defining new acceptability-based extensions, and (iii) establishing user postures to analyze the argumentation framework where special constraints are satisfied, In previous works by Budán et al. $(2014,2016)$, we presented preliminary results related to this line of research; here, we improve previous semantic definitions, present an algorithm to perform the labeling process, and analyze the set of postulates that satisfy the semantics presented in this formalism. For now, we restrict our approach to consider well-founded BAFs, where no cycles-including self-attacking and self-supporting arguments-exist.

This paper is structured as follows: Section 2 introduces an example to motivate the usefulness of our formalization in the context of social platforms. Then, in Section 3, we present a brief introduction to $B A F$; next, Section 4 introduces a refined version of the abstract algebra presented by Budán et al. $(2014,2016)$ for handling the labels associated with arguments in the argumentation domain; in Section 5 and Section 6, we present an extension of BAF to represent argument attributes, and how these attributes are affected by the interactions between the arguments. Furthermore, we present the algorithms to perform the labeling process. In Section 7, the acceptability process based on argument's features is presented, while in Section 8 we study the postulates satisfied by our proposal; Section 9 develops the running example from the social platforms domain, applying our formalism to analyze the features associated with each argument presented in a Twitter-like discussion. Finally, Section 10 discusses related work, and Section 11 presents our conclusions and describes future research directions.

As supplementary material, we present two issues that we will further develop in future lines of research. In Appendix A, we determine special coefficients associated with the argumentation framework that represent the effectiveness of the support and conflict relation, as well as a refinement process to improve the argumentative discussion by avoiding analysis of less relevant arguments. In Appendix B, we present a preliminary analysis of the problem of dealing with cycles in our system. Finally, we include proofs of all results in Appendix C.

\section{Running Example}

We now introduce a running example showing how our approach could help to achieve an improved analysis, as discussed in the previous section. Consider a scenario involving a discussion by a group of parents on Twitter (or a similar social platform) analyzing the adequacy of a school for a child. In order to reach a decision, they evaluate arguments according to: $(i)$ their preferences, assigning to each of them an assessment of relevance, and (ii) a degree of "social rating" associated with each argument, describing how popular the argument is in the discussion. Arguments A through $\mathrm{K}$ are obtained from the tweets depicted in Figure 1; we assume that the social rating can be derived from the tweet's performance (retweets, likes, and comments), which is shown on the right.

This example illustrates how the knowledge used to solve a specific problem can be naturally structured as arguments, and that considering the relationships among these arguments is useful in reaching a decision. Also, since the parents analyze the arguments in favor and against the 


\begin{tabular}{|c|c|c|}
\hline $\begin{array}{l}\text { Jessica @jesshull79 . Jun } 23 \\
\text { School @AilenEdu has a good approach to teaching } \\
\text { and provides students with excellent material to boost } \\
\text { their learning capabilities. }\end{array}$ & $\begin{array}{l}\text { Re-Tweets: } \\
\text { Likes: } \\
\text { Comments: }\end{array}$ & $\begin{array}{l}7 \\
32 \\
5\end{array}$ \\
\hline $\begin{array}{l}\text { Timothy @smithtim999 . Jun } 23 \\
\text { I'm worried that many students drop out of } \\
\text { @AilenEdu. What are the root causes of this? }\end{array}$ & $\begin{array}{l}\text { Re-Tweets: } \\
\text { Likes: } \\
\text { Comments: }\end{array}$ & $\begin{array}{l}3 \\
12 \\
4\end{array}$ \\
\hline $\begin{array}{l}\text { Scott @sbivensny · Jun } 24 \\
\text { It's clear from continuing education polls that } \\
\text { @AilenEdu doesn't provide opportunities for all } \\
\text { students to obtain a college education. }\end{array}$ & $\begin{array}{l}\text { Re-Tweets: } \\
\text { Likes: } \\
\text { Comments: }\end{array}$ & $\begin{array}{l}9 \\
41 \\
7\end{array}$ \\
\hline $\begin{array}{l}\text { Sylvia @sylviag1980.Jun } 24 \\
\text { Teachers at @AilenEdu are not always fair in their } \\
\text { treatment of students. }\end{array}$ & $\begin{array}{l}\text { Re-Tweets: } \\
\text { Likes: } \\
\text { Comments: }\end{array}$ & $\begin{array}{l}0 \\
5 \\
1\end{array}$ \\
\hline $\begin{array}{l}\text { Leslie @lesguev00 . Jun } 24 \\
\text { From what l've seen, teachers at @AilenEdu don't } \\
\text { have the skills to address the new generation of } \\
\text { students. }\end{array}$ & $\begin{array}{l}\text { Re-Tweets: } \\
\text { Likes: } \\
\text { Comments: }\end{array}$ & $\begin{array}{l}5 \\
16 \\
8\end{array}$ \\
\hline $\begin{array}{l}\text { Dorothy @dorokendrick.Jun } 24 \\
\text { Students at @AilenEdu generally enjoy the school's } \\
\text { approach to teaching. }\end{array}$ & $\begin{array}{l}\text { Re-Tweets: } \\
\text { Likes: } \\
\text { Comments: }\end{array}$ & $\begin{array}{l}5 \\
21 \\
3\end{array}$ \\
\hline $\begin{array}{l}\text { Michael @mikerichardson · Jun } 24 \\
\text { @AilenEdu has a strong offering of core academic } \\
\text { subjects like English, History, Math, Science, Arts, } \\
\text { and Foreign Languages. }\end{array}$ & $\begin{array}{l}\text { Re-Tweets: } \\
\text { Likes: } \\
\text { Comments: }\end{array}$ & $\begin{array}{l}15 \\
42 \\
19\end{array}$ \\
\hline $\begin{array}{l}\text { Emily @gibsonem · Jun } 25 \\
\text { Classes at @AilenEdu are very large, and have an } \\
\text { extensive extracurricular activity program after school } \\
\text { and on weekends. }\end{array}$ & $\begin{array}{l}\text { Re-Tweets: } \\
\text { Likes: } \\
\text { Comments: }\end{array}$ & $\begin{array}{l}12 \\
8 \\
16\end{array}$ \\
\hline $\begin{array}{l}\text { David @davesuber81.Jun } 25 \\
\text { @AilenEdu takes interest in each student's learning, } \\
\text { and provides opportunities for children to get extra } \\
\text { help when needed. }\end{array}$ & $\begin{array}{l}\text { Re-Tweets: } \\
\text { Likes: } \\
\text { Comments: }\end{array}$ & $\begin{array}{l}8 \\
21 \\
3\end{array}$ \\
\hline $\begin{array}{l}\text { Dennis @dstafford } 000 \text {.Jun } 25 \\
\text { Students at @AilenEdu appear to be courteous, } \\
\text { happy, and disciplined. }\end{array}$ & $\begin{array}{l}\text { Re-Tweets: } \\
\text { Likes: } \\
\text { Comments: }\end{array}$ & $\begin{array}{l}2 \\
18 \\
3\end{array}$ \\
\hline $\begin{array}{l}\text { Kristen @kriscordero1.Jun } 26 \\
\text { I heard@AilenEdu will close an agreement with } \\
\text { several universities so that students have more } \\
\text { options when they graduate. }\end{array}$ & $\begin{array}{l}\text { Re-Tweets: } \\
\text { Likes: } \\
\text { Comments: }\end{array}$ & $\begin{array}{l}19 \\
26 \\
10\end{array}$ \\
\hline
\end{tabular}

Figure 1: Tweets posted by people regarding a school's characteristics. The argument names on the left will be used throughout the paper as our running example. The tweets' performance information on the right will be used later on to derive argument valuations.

school selection according to their preferences and social rating, the arguments endorsing specific characteristics show the usefulness of representing additional information.

Users can have different views of the real-world, where uncertainty is always present. Therefore, the features associated with the arguments may not be fully specified, but it may still be possible to define a spectrum representing the specific user's abilities, points of view, or biases. For example, the social ranking of an argument can oscillate due to comments for and against it, as well as by like and re-tweet counts ( $c f$. Figure 2). In this work, we annotate each attribute of an argument with an interval $[x, y]$ to represent the variation of a particular attribute's value produced by the 


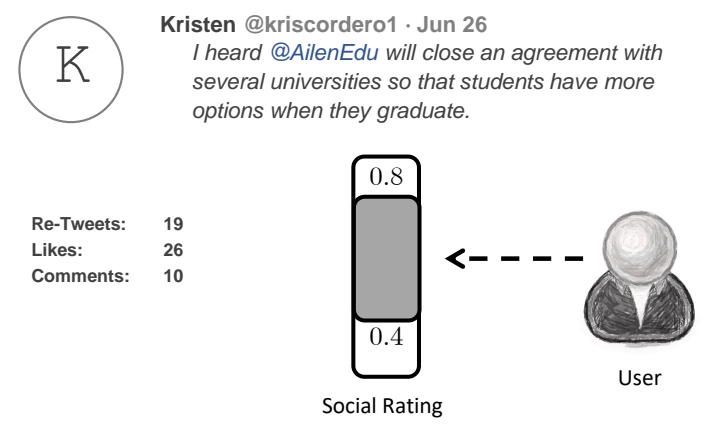

Figure 2: According to this user's perception, this argument's valuation is between 0.4 and 0.8 , taking into account the social rating (tweet author, metrics, etc.).

tolerance of the user's preference or their perception uncertainty. It is important to remark that the process of identifying relevant tweets, and obtaining arguments from them, is out of the scope of this paper; however, the Natural Language Processing (Belinkov \& Glass, 2019) and Argument Mining (Lippi \& Torroni, 2016) communities have research lines proposing tools to address this task with promising results that are continually improved-we refer the interested reader to the literature for more details.

The interactions between arguments can be given as support (e.g., the positive relation between $\mathrm{F}$ and $\mathrm{A}$ ) or as conflict (e.g., the negative relation between B and A). Each pro and con argument is associated with descriptive features that have a weight in the final decision. That is, these features describe the quality of arguments that can be affected by the interactions between arguments, such as their support and conflict. These changes in the quality of the arguments can give clues to their effectiveness in the role that they play in the argumentative process. Thus, actions such as attacks and supports will be directly reflected in the final quality of the arguments.

\section{Bipolar Abstract Argumentation}

The basic approach to abstract argumentation-based reasoning is to consider arguments for and against a conclusion, analyze the general scenario, and then select the acceptable arguments. In the argumentation domain, arguments have different roles with respect to each other; it is possible to say that arguments are presented in a bipolar way since those in favor of a conclusion can be considered as positive while those against the conclusion as negative. Based on this intuition, when representing the essential argumentation mechanism, the notion of bipolarity is a natural one. Abstracting away from the inner structure of the arguments, the $B A F$ proposed by (Cayrol \& Lagasquie-Schiex, 2005b) extends Dung's notion of acceptability in abstract argumentation frameworks by distinguishing two independent forms of interaction between arguments: support and attack. The relation of support is assumed to be independent of the relation of attack, providing the former a positive relation between arguments.

Definition 1 (Bipolar Argumentation Framework) A Bipolar Argumentation Framework (BAF) is a 3-tuple $\Theta=\left\langle\mathrm{AR}, \mathcal{R}_{a}, \mathcal{R}_{s}\right\rangle$, where $\mathrm{AR}$ is a set of arguments, and the elements $\mathcal{R}_{a}$ and $\mathcal{R}_{s}$ are disjoint binary relations on AR called attack and support, respectively. 
In $B A F$, the graph description introduced by Dung (1995) is extended by adding the representation of support between arguments. This approach provides a starting point for enriching the analysis of discussions with the natural bipolarity of human reasoning. Additionally, Cayrol et al. (2005b) introduce the notions of supported and secondary defeat, which combine a sequence of supports with a direct defeat to consider the interaction between supporting and defeating arguments.

Definition 2 (Defeat in $B A F$ ) Let $\Theta=\left\langle\mathrm{AR}, \mathcal{R}_{a}, \mathcal{R}_{s}\right\rangle$ be a $B A F$, and $\mathrm{A}, \mathrm{B} \in \mathrm{AR}$ be two arguments. Then, we will say that:

- A is a supported defeat for $\mathrm{B}$ iff there exists a sequence $\mathrm{A}_{1} R_{1} \ldots R_{n} \mathrm{~A}_{n+1}$, with $n \geq 1$, where $\mathrm{A}_{1}=\mathrm{A}$ and $\mathrm{A}_{n+1}=\mathrm{B}$, such that $\forall i=1, \ldots, n-1, R_{i} \in \mathcal{R}_{s}$ and $R_{n}=\mathcal{R}_{a}$.

- A is a secondary defeat for $\mathrm{B}$ iff there exists a sequence $\mathrm{A}_{1} R_{1} \ldots R_{n} \mathrm{~A}_{n+1}$, with $n \geq 2$, where $\mathrm{A}_{1}=\mathrm{A}$ and $\mathrm{A}_{n+1}=\mathrm{B}$, such that $R_{1}=\mathcal{R}_{a}$ and $\forall i=2, \ldots, n, R_{i} \in \mathcal{R}_{s}$.

Also, in any BAF, a sequence reduced to two arguments $\mathrm{A} \mathcal{R}_{a} \mathrm{~B}$ (a direct defeat $\mathrm{A} \rightarrow \mathrm{B}$ ) is also considered as a supported defeat from A to $\mathrm{B}$.

Cayrol and Lagasquie-Schiex (2005b) argue that a set of arguments must be in some sense coherent to model one side of an intelligent dispute. The coherence of a set of arguments is analyzed internally (a set of arguments in which an argument attacks another in the same set is not acceptable), and externally (a set of arguments that contains both a supporter and an attacker for the same argument is not acceptable). Internal coherence is captured by extending the definition of conflict-free set proposed by (Dung, 1995), while external coherence is captured via the notion of safe set.

Definition 3 (Conflict-Freeness and Safety) Let $\Phi=\left\langle\mathrm{AR}, \mathcal{R}_{a}, \mathcal{R}_{s}\right\rangle$ be a BAF and $S \subseteq \mathrm{AR}$ be a set of arguments. Then: ( $i) S$ is conflict-free iff $\nexists \mathrm{A}, \mathrm{B} \in S$ such that there is a supported or secondary defeat from $\mathrm{A}$ to $\mathrm{B}$; and (ii) $S$ is safe iff $\nexists \mathrm{A} \in \mathrm{AR}$ and $\nexists \mathrm{B}, \mathrm{C} \in S$ such that there is a supported or secondary defeat from $\mathrm{B}$ to $\mathrm{A}$, and either there is a sequence of supports from $\mathrm{C}$ to $\mathrm{A}$, or $\mathrm{A} \in S$.

The notion of conflict-freeness requires taking supported and secondary defeats into account, becoming a more restrictive definition than the classical version of conflict-freeness originally proposed by Dung. In addition, the notion of safety was shown to be powerful enough to encompass conflict-freeness. The closure under $\mathcal{R}_{s}$, which concerns only the support relation, was also considered by (Cayrol \& Lagasquie-Schiex, 2005b).

Definition 4 (Closure in $B A F$ ) Let $\Phi=\left\langle A R, \mathcal{R}_{a}, \mathcal{R}_{s}\right\rangle$ be a BAF and $S \subseteq \mathrm{AR}$ be a set of arguments. $S$ is closed under $\mathcal{R}_{s}$ iff $\forall \mathrm{A} \in S, \forall \mathrm{B} \in \mathrm{AR}$, if $\mathrm{A} \mathcal{R}_{s} \mathrm{~B}$ then $\mathrm{B} \in S$.

Based on the previous concepts, the notion of defense for an argument with respect to a set of arguments is extended by taking into account the relations of support and conflict between arguments.

Definition 5 (Defense for A by $S$ ) Let $\Phi=\left\langle\mathrm{AR}, \mathcal{R}_{a}, \mathcal{R}_{s}\right\rangle$ be a $B A F, S \subseteq \mathrm{AR}$ be a set of arguments and $\mathrm{A} \in \mathrm{AR}$ be an argument. $S$ collectively defends $\mathrm{A}$ iff $\forall \mathrm{B} \in \mathrm{AR}$, if $\mathrm{B}$ is a supported or secondary defeat of $\mathrm{A}$ then $\exists \mathrm{C} \in S$ such that $\mathrm{C}$ is a supported or secondary defeat of $\mathrm{B}$. In this case, it can be interpreted that $\mathrm{C}$ defends $\mathrm{A}$ from $\mathrm{B}$. 


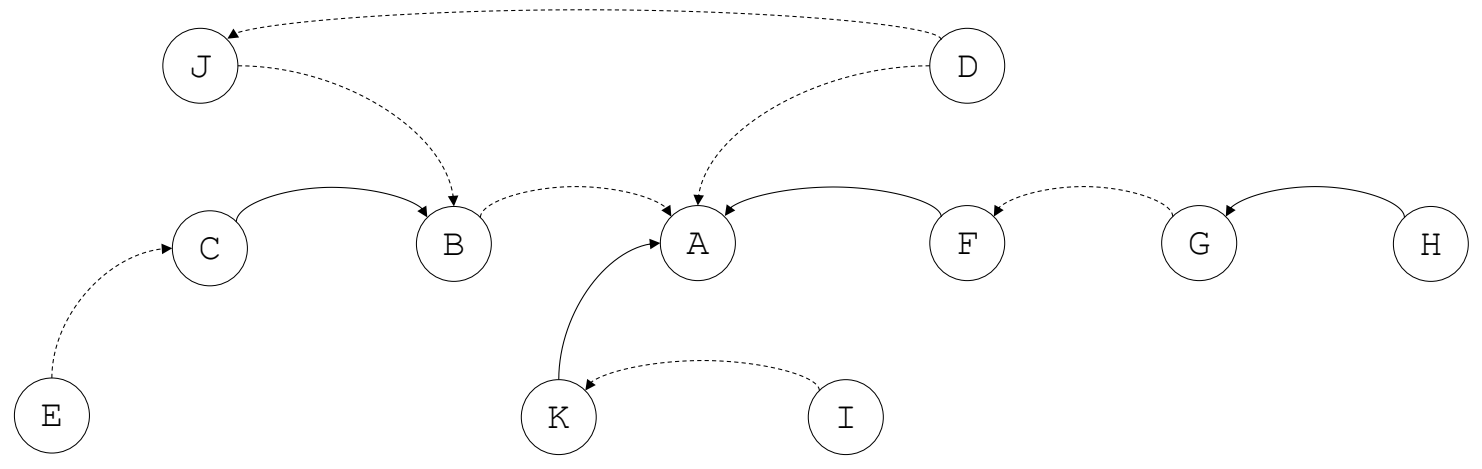

Figure 3: Initial bipolar argumentation graph.

Three different definitions for admissibility were proposed, from the most general to the most specific. The most general is based on Dung's definition; the notion of s-admissibility takes into account external coherence, and external coherence is strengthened by requiring that admissible sets be closed for $\mathcal{R}_{s}$.

Definition 6 (Admissibility Notions in BAF) Let $\Phi=\left\langle\mathrm{AR}, \mathcal{R}_{a}, \mathcal{R}_{s}\right\rangle$ be a BAF and $S \subseteq \mathrm{AR}$ be a set of arguments. The admissibility of a set $S$ is defined as follows: $i) S$ is d-admissible if $S$ is conflictfree and defends all its elements; ii) $S$ is s-admissible if $S$ is safe and defends all its elements; and iii) $S$ is c-admissible if $S$ conflict-free, closed for $\mathcal{R}_{s}$ and defends all its elements.

From the previous notions, Cayrol and Lagasquie-Schiex (2005b) proposed different semantics for computing argument acceptability. These semantics consider the previous admissibility notion, redefining the classical ones proposed by (Dung, 1995).

Definition 7 (Preferred Extensions in $B A F$ ) Let $\Theta=\left\langle\mathrm{AR}, \mathcal{R}_{a}, \mathcal{R}_{s}\right\rangle$ be a $B A F$, and $S \subseteq \mathrm{AR}$ be a set of arguments. $S$ is a d-preferred (resp. s-preferred, c-preferred) extension if $S$ is maximal (for set-inclusion) among the d-admissible (resp. s-admissible, c-admissible) subsets of AR.

Example 1 Let $\Phi=\left\langle\mathrm{AR}, \mathcal{R}_{a}, \mathcal{R}_{s}\right\rangle$ be a BAF, composed as follows (cf. Figure 3):

$$
\begin{aligned}
& \mathrm{AR}=\{\mathrm{A}, \mathrm{B}, \mathrm{C}, \mathrm{D}, \mathrm{E}, \mathrm{F}, \mathrm{G}, \mathrm{H}, \mathrm{I}, \mathrm{J}, \mathrm{K}\}, \\
& \mathcal{R}_{a}=\{(\mathrm{K}, \mathrm{A}) ;(\mathrm{F}, \mathrm{A}) ;(\mathrm{C}, \mathrm{B}) ;(\mathrm{H}, \mathrm{G})\} \text {, and } \\
& \left.\mathcal{R}_{s}=\{(\mathrm{B}, \mathrm{A}) ;(\mathrm{J}, \mathrm{B}) ;(\mathrm{E}, \mathrm{C})) ;(\mathrm{D}, \mathrm{A}) ;(\mathrm{D}, \mathrm{J}) ;(\mathrm{G}, \mathrm{F}) ;(\mathrm{I}, \mathrm{K})\right\} .
\end{aligned}
$$

We analyze the bipolar argumentation framework $\Phi$ characterized by the bipolar interaction graph depicted in Figure 3; As usual, solid lines represent the attack relation, while dotted lines represent the support relation. For instance, $\mathrm{E}$ is a support defeat of $\mathrm{B}$, since $\mathrm{E}$ supports $\mathrm{C}$, and $\mathrm{B}$ is attacked by $\mathrm{C}$ (direct attacker); in addition, $\mathrm{C}$ is a secondary defeat of $\mathrm{A}$, because $\mathrm{B}$ supports $\mathrm{A}$, which is attacked by $\mathrm{C}$. On the other hand, $\mathrm{F}$ is a direct attacker of $\mathrm{A}$; however, $\mathrm{H}$ is a secondary defeat of $\mathrm{F}$, because $\mathrm{G}$ supports $\mathrm{F}$, which is attacked by $\mathrm{H}$. Thus, the attack from $\mathrm{F}$ to $\mathrm{A}$ is invalidated. Finally, I is a support defeat of A, since I supports K, and A is attacked by $\mathrm{K}$ (direct attacker).

Analyzing BAF $\Phi$ (cf. Figure 3) using the classical bipolar notions, the set $S_{1}=\{\mathrm{I}, \mathrm{K}, \mathrm{E}, \mathrm{C}, \mathrm{H}\}$ is $d$-admissible, s-admissible, and c-admissible, while the set $S_{2}=\{\mathrm{I}, \mathrm{K}, \mathrm{E}, \mathrm{C}, \mathrm{D}, \mathrm{J}, \mathrm{H}\}$ is d-admissible, 
but not s-admissible ( $\mathrm{C}$ is an attacker of $\mathrm{B}$, which is supported by $\mathrm{J}$ ), and not c-admissible (D supports A, which does not belong to $S_{2}$ ).

Bipolar argumentation frameworks typically focus on computing an acceptable set of arguments based on a specific analysis that considers only the support and conflict relations between the arguments involved in a discussion. The initial approach to bipolarity in argumentation frameworks was introduced by Amgoud et al. (2004), Cayrol and Lagasquie (2005a, 2009, 2013), and the survey by Cohen et al. (2014a) - these works will be discussed in Section 10.

\section{Algebra of Argumentation Labels}

The process of abstraction is generally used in many disciplines-and in particular mathematics and computer science - to focus interest on what is relevant for a particular purpose. Thus, by abstracting away details, we obtain conceptual generality that is useful for a specific purpose. On the one hand, in computer science, researchers have created different abstract argumentation formalisms used to model a rational discourse to compute a set of acceptable arguments, abstractly representing the entities and the reasons in favor or against a specific action, decision, or recommendation. In this sense, these formalisms place special attention on the (positive and negative) relations between the abstract arguments, without considering the logical structure behind them. On the other hand, mathematicians have created theories with various structures that apply to many objects; for instance, abstract algebra evolved from earlier forms of arithmetic and reached its potential by the process of successive abstractions that allowed to obtain increasingly complex systems without losing mathematical purity and "inherent beauty". The axiomatic nature of abstract algebra deals with systems whose elements are of an unspecified type, together with specific operations that satisfy a prescribed list of axioms or postulates.

In our formalism, the use of labels gives us the possibility of representing distinctive features of arguments, and these labels change according to the existing relations between arguments. Following this idea, we use an algebrization that consists of a set of labels equipped with a collection of operators to be used in combining and propagating the labels according to argument interactions (Budán et al., 2015) - labels must represent information about the arguments and how they interact. The algebra is based on an ordered set, allowing the comparison of labels; this set is also characterized in an abstract way to allow the adaptation to different applications. A natural way of representing this information is to use a scale that measures a particular feature of the argument. We will consider valuations ranging between two distinguished elements: $T$ and $\perp$, where $\perp$ represents the least possible degree in which an argument may possess a particular attribute, and $T$ the maximum. To operate with these elements, we borrow operations from fuzzy logic (Zimmermann, 2001), a well-studied set of tools for making inferences when the available information is imprecise rather than exact.

Definition 8 (Algebra of Argumentation Labels) An algebra of argumentation labels is a 8-tuple of the form $\mathrm{A}=\langle\mathfrak{L}, \leq, \odot, \otimes, \oplus, \ominus, \top, \perp\rangle$, where:

- $\mathfrak{L}$ is a set of labels called the domain of labels.

$-\leq$ is a partial order over $\mathfrak{L}$ (that is, a reflexive, antisymmetric, and transitive relation). 


\section{LABELED Bipolar ARguMENTATION FrAMEWORKS}

- $\top$ and $\perp$ are two distinguished elements of $\mathfrak{L}$ such that $\forall \alpha \in \mathfrak{L}, \alpha \leq \top$, i.e., $\top$ is the last label with respect to $\leq$, and $\forall \alpha \in \mathfrak{L}, \perp \leq \alpha$, i.e., $\perp$ is the first.

- $\odot: \mathfrak{L} \times \mathfrak{L} \rightarrow \mathfrak{L}$ is called a support accumulation operation and satisfies:

$\odot$ is commutative: $\forall \alpha, \beta \in \mathfrak{L}, \alpha \odot \beta=\beta \odot \alpha$.

$\odot$ is monotone: $\forall \alpha, \beta, \gamma \in \mathfrak{L}$, if $\alpha \leq \beta$, then $\alpha \odot \gamma \leq \beta \odot \gamma$.

$\odot$ is associative: $\forall \alpha, \beta, \gamma \in \mathfrak{L}, \alpha \odot(\beta \odot \gamma)=(\alpha \odot \beta) \odot \gamma$.

$\top$ is the neutral element for $\odot: \forall \alpha \in \mathfrak{L}, \alpha \odot \top=\alpha$.

$\perp$ is the absorbing element for $\odot: \forall \alpha \in \mathfrak{L}, \alpha \odot \perp=\perp$.

- $\otimes: \mathfrak{L} \times \mathfrak{L} \rightarrow \mathfrak{L}$ is called an attack accumulation operation that satisfies:

$\otimes$ is commutative: $\forall \alpha, \beta \in \mathfrak{L}, \alpha \otimes \beta=\beta \otimes \alpha$.

$\otimes$ is monotone: $\forall \alpha, \beta, \gamma \in \mathfrak{L}$, if $\alpha \leq \beta$, then $\alpha \otimes \gamma \leq \beta \otimes \gamma$.

$\otimes$ is associative: $\forall \alpha, \beta, \gamma \in \mathfrak{L}, \alpha \otimes(\beta \otimes \gamma)=(\alpha \otimes \beta) \otimes \gamma$.

$\top$ is the neutral element for $\otimes: \forall \alpha \in \mathfrak{L}, \alpha \otimes \top=\alpha$.

$\perp$ is the absorbing element for $\otimes: \forall \alpha \in \mathfrak{L}, \alpha \otimes \perp=\perp$.

- $\oplus: \mathfrak{L} \times \mathfrak{L} \rightarrow \mathfrak{L}$ is called a strengthening operation that satisfies:

$\oplus$ is commutative: $\forall \alpha, \beta \in \mathfrak{L}, \alpha \oplus \beta=\beta \oplus \alpha$.

$\oplus$ is monotone: $\forall \alpha, \beta, \gamma \in \mathfrak{L}$, if $\alpha \leq \beta$, then $\alpha \oplus \gamma \leq \beta \oplus \gamma$.

$\oplus$ is associative: $\forall \alpha, \beta, \gamma \in \mathfrak{L}, \alpha \oplus(\beta \oplus \gamma)=(\alpha \oplus \beta) \oplus \gamma$.

$\perp$ is the neutral element for $\oplus: \forall \alpha \in \mathfrak{L}, \alpha \oplus \perp=\alpha$.

$\top$ is the absorbing element for $\oplus: \forall \alpha \in \mathfrak{L}, \alpha \oplus \top=\top$.

- $\ominus: \mathfrak{L} \times \mathfrak{L} \rightarrow \mathfrak{L}$ is called $a$ weakening operation that satisfies:

$\ominus$ is non-commutative: $\exists \alpha, \beta \in \mathfrak{L}, \alpha \ominus \beta \neq \beta \ominus \alpha$.

$\ominus$ is monotone: $\forall \alpha, \beta, \gamma \in \mathfrak{L}$, if $\alpha \leq \beta$, then $\alpha \ominus \gamma \leq \beta \ominus \gamma$.

$\ominus$ is non-associative: $\exists \alpha, \beta, \gamma \in \mathfrak{L}, \alpha \ominus(\beta \ominus \gamma) \neq(\alpha \ominus \beta) \ominus \gamma$.

$\perp$ is the neutral element for $\ominus: \forall \alpha \in \mathfrak{L}, \alpha \ominus \perp=\alpha$.

$\forall \alpha, \beta \in \mathfrak{L}, \alpha \ominus \beta \leq \alpha$ if $\beta<\alpha$.

$\forall \alpha, \beta \in \mathfrak{L}, \alpha \ominus \beta=\perp$ if $\beta \geq \alpha$; In particular, $\forall \alpha \in \mathfrak{L}, \alpha \ominus \top=\perp$.

$\forall \alpha, \beta \in \mathfrak{L}$, if $\alpha \ominus \beta=\perp$ and $\beta \ominus \alpha=\perp$, then $\alpha=\beta$.

$\forall \alpha, \beta \in \mathfrak{L}$, if $(\alpha \oplus \beta)<\top$, then $((\alpha \oplus \beta) \ominus \beta)=\alpha$. 


\subsection{Support and Attack Accumulation Operations}

The support accumulation operation, denoted with $\odot$, is used to determine the valuations that enforce a single argument based on the supporting arguments' strength. It is clear that the result of this operation should be invariant with respect to the order in which the supporting arguments are considered, and therefore the operation is both commutative and associative, with $T$ as the neutral element. Furthermore, if stronger arguments support an argument, its support accumulation valuation must be higher than that of one with supporters with lesser valuations, establishing monotonicity. Also, $\perp$ is the absorbing element for $\odot$, since the result of combining $\perp$ with any element of the set $\mathfrak{L}$ is the absorbing element itself.

The attack accumulation operation, denoted with $\otimes$, is used to determine the valuations that weaken a single argument based on the attacking arguments' strength. As before, the result of this operation should be invariant with respect to the order in which the attacking arguments are considered, and therefore the operation is both commutative and associative, with $T$ as the neutral element. Furthermore, if stronger arguments attack an argument, its attack accumulation valuation must be higher than that of one with attacker with lesser valuations, establishing monotonicity. In addition, $\perp$ is the absorbing element for $\otimes$, since the result of combining $\perp$ with any element of the set $\mathfrak{L}$ is the absorbing element itself.

\subsection{Strengthening and Weakening Operations}

The strengthening operation, denoted with $\oplus$, determines the enforced valuation associated with an argument; i.e., the accumulation valuation is combined with the original valuation associated with the object argument through the strengthening operator (cf. Figure 4). Naturally, a way of doing this would be to directly add these valuations, taking into consideration the conditions imposed by the domain. For this reason, the $\oplus$ operation has some of the properties of addition of real numbersit is commutative and associative, with $\perp$ (the least possible evaluation) as the neutral element, i.e., arguments with valuation $\perp$ not produced any effect. Furthermore, the $\oplus$ operation satisfies monotonicity, ensuring that the valuation of a conclusion does not decrease if the support valuation increases. Also, $T$ is the absorbing element for $\oplus$ since it represents the highest possible valuation. In Figure 4, the support accumulation operator is instantiated as $\alpha \odot \beta=\min (\alpha, \beta)$ (Gödel t-norm) while the strengthening operator is established as $\alpha \oplus \beta=\min (\alpha+\beta, 1)$ (bounded sum c-norm).

The weakening operation, denoted with $\ominus$, determines the overall valuation of an argument considering the strength associated with opposing reasons (cf. Figure 5). This operator enjoys the monotonicity property, ensuring that the valuation of a conclusion decreases if the valuation of the reasons against such a conclusion increases. Furthermore, $\perp$ is the neutral element for $\ominus$, specifying that the valuation associated with an argument is not affected by a counterargument with valuation equal to the least possible valuation (a valuation that represents no strength). On the other hand, in the opposite sense, an attacker with valuation equal to the highest possible valuation $\top$ completely neutralizes the strength of the attacked argument. Coming back to Figure 4, the attack accumulation operator is instantiated as $\alpha \odot \beta=\alpha \beta$ (minimum t-norm) while the weakening operator is established as $\alpha \ominus \beta=\max (\alpha-\beta, 0)$.

It is interesting to observe that the accumulation and strengthening operators can be instantiated with a function from the t-conorm family (Schweizer \& Sklar, 1961, 1963). These functions are used in different application domains; for example, Dubois et al. (1982) present different ways to 


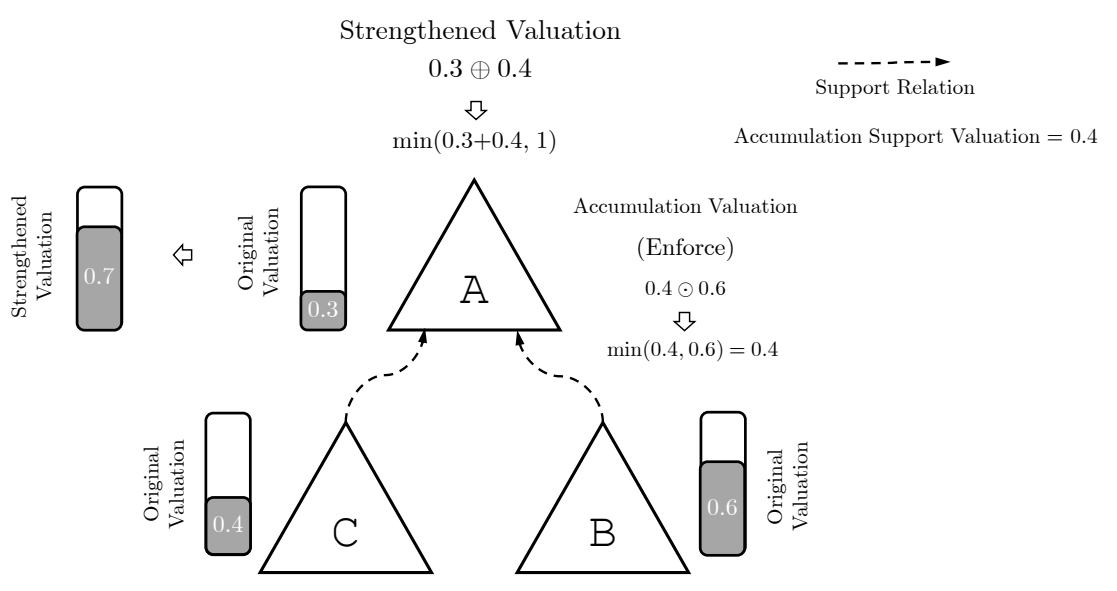

Figure 4: Combination of the original valuation and the impact of supports: behavior of the $\oplus$ operator in the Algebra of Argumentation Labels.

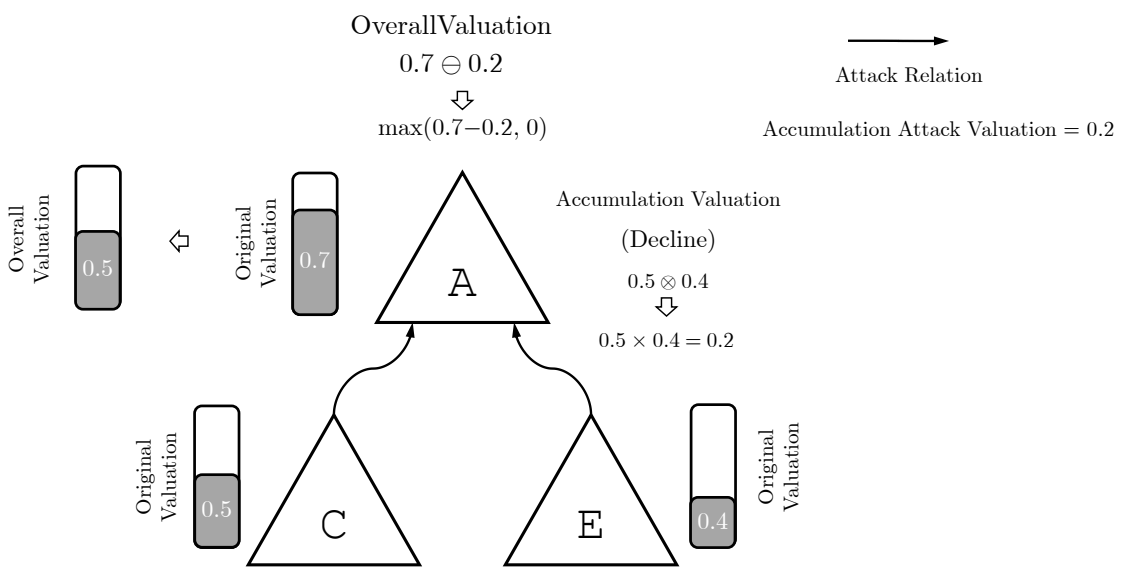

Figure 5: Combination of the original valuation and the impact of attacks: behavior of the $\ominus$ operator in the Algebra of Argumentation Labels.

perform aggregation of arguments based on user preference through t-conorms. Another example is the work of Lukasiewicz et al. (2008), which introduces a series of criteria to perform aggregation of arguments supporting a particular conclusion in decision-support systems, taking into account the uncertainty level associated with these arguments. On the other hand, weakening operators are used in different application domains. Examples of this are the works of Budán et al. $(2015,2018)$; the former presents a structured argumentation formalism where the weakening operator is used to handle the relevance associated with the information in the legal domain, while the latter presents a formalism with a weakening operator that considers a temporal dimension establishing the time intervals in which an argument is defeated.

No further assumptions are made regarding the postulates adopted in Definition 8 for the operators. For example, the weakest link principle could be the basis of the support accumulation operator, but this is not the only possibility. Another example: given that $\perp$ is the absorbing ele- 
ment, support can be related to what in argument maps are called dependent premises. Thus, the meaning of each instance of these operators will depend on the application domain and the behavior that the user wants to model. In future work, we will analyze how the definition of these operations can be expanded to model the different interpretations associated with the support and conflict relations in the context of bipolar frameworks (Nouioua \& Risch, 2010; Cayrol \& Lagasquie-Schiex, 2013).

Finally, another possibility supported in this formalism is handling second-order uncertainty; instead of labels taking numeric values, we can have intervals representing uncertainty over the specific value associated with the underlying property. In this case, the operators in the algebra must be defined so that intervals are adequately manipulated, a concept that was addressed by Budán et al. (2015).

In what follows, we present our formalism, where the algebra of argumentation labels has an important role. The operators described above allow us to represent intuitively correct behaviors when handling the labels in the argumentation process; we show that this is the case by proving that the formalism satisfies a set of reasonable properties, which we present as postulates.

\section{Labeled Bipolar Argumentation Frameworks}

Even though $B A F s$ allow to model support and attack, they lack the mechanisms to represent any additional argument features. To address this, we extend $B A F s$ by incorporating labels that hold specific information given about each argument and the result of the interactions among them. These labels extend the argumentation framework's representational capabilities and provide ways of redefining the acceptability process. Previous works by Budán et al. $(2014,2016)$ presented an early version of this formalism; now, continuing its development, we improve previous semantic definitions, present an algorithm to perform the labeling process, and analyze a set of postulates that are satisfied by the semantics.

Definition 9 (Labeled Bipolar Argumentation Framework) A Labeled Bipolar Argumentation Framework $(L-B A F)$ is a 3-tuple $\Psi=\langle\Theta, \mathbf{A}, v\rangle$, where $\Theta=\left\langle\mathrm{AR}, \mathcal{R}_{a}, \mathcal{R}_{s}\right\rangle$ is the underlying bipolar argumentation framework, $\mathbf{A}$ is a set of algebras of argumentation labels $\mathrm{A}_{1}, \mathrm{~A}_{2}, \ldots, \mathrm{A}_{n}$ (one for each feature represented by the labels), and $v$ is a function that assigns to each element of $A R$ an $n$-tuple of elements in the algebras $\mathrm{A}_{i}, i=1, \ldots, n$. That is, $v: \mathrm{AR} \longrightarrow\left|\mathrm{A}_{1}\right| \times\left|\mathrm{A}_{2}\right| \times \ldots \times\left|\mathrm{A}_{n}\right|$, where $\left|\mathrm{A}_{i}\right|$ is the label domain of the algebra $\left|\mathrm{A}_{i}\right|, 1 \leq i \leq n{ }^{1}$

Notation: Given $\mathrm{A} \in \mathrm{AR}$, the set $\left\{\mathrm{X} \in \mathrm{AR} \mid \mathrm{X} \mathcal{R}_{a} \mathrm{~A}\right\}$ is denoted with $\overrightarrow{(\mathrm{A})}$, and the set $\left\{\mathrm{X} \in \mathrm{AR} \mid \mathrm{X} \mathcal{R}_{s} \mathrm{~A}\right\}$ is denoted with $(\vec{A})$.

Next, we present an example that accompanies the development of this formalism with the aim of clarifying the concepts introduced in turn.

Example 2 Let $\Psi=\langle\Theta, \mathbf{A}, v\rangle$ be an $L-B A F$, composed as follows:

$$
\mathrm{AR}=\{\mathrm{A}, \mathrm{B}, \mathrm{C}, \mathrm{D}, \mathrm{E}, \mathrm{F}, \mathrm{G}, \mathrm{H}, \mathrm{I}, \mathrm{J}, \mathrm{K}\},
$$

1. When no confusion may occur, we follow the usual convention of mentioning elements in an algebra $A$ instead of referring to elements in its corresponding carrier set $|\mathrm{A}|$. 
$\mathcal{R}_{a}=\{(\mathrm{K}, \mathrm{A}) ;(\mathrm{F}, \mathrm{A}) ;(\mathrm{C}, \mathrm{B}) ;(\mathrm{H}, \mathrm{G})\}$,
$\mathcal{R}_{s}=\{(\mathrm{B}, \mathrm{A}) ;(\mathrm{J}, \mathrm{B}) ;(\mathrm{E}, \mathrm{C}) ;(\mathrm{D}, \mathrm{A}) ;(\mathrm{D}, \mathrm{J}) ;(\mathrm{G}, \mathrm{F}) ;(\mathrm{I}, \mathrm{K})\}$,

$\mathbf{A}=\{\mathrm{A}, \mathrm{B}\}$ is the set of algebras representing accuracy level and reliability strength for each argument in $\mathrm{AR}$. These algebras are defined as follows:

The domain of labels $\mathfrak{L}_{1}$ of algebra $\mathrm{A}$ is the real interval $[0,1]$ representing a normalized accuracy valuation with $T=1$ and $\perp=0$. The accuracy of the arguments represents the certainty level associated with the information proposed by the argument. The accumulation, strengthening, and weakening operators are defined as follows:

\begin{tabular}{|c|c|c|}
\hline \multicolumn{3}{|c|}{ Accuracy } \\
\hline$\gamma \odot \beta=\min (\gamma, \beta)$ & & $\begin{array}{l}\text { The support accumulation operator } \\
\text { models the support valuation as the } \\
\text { minimal accuracy valuations associ- } \\
\text { ated with the supporting arguments; } \\
\text { thus, the support valuation that en- } \\
\text { forces an argument is as good as the } \\
\text { worst argument that support it. }\end{array}$ \\
\hline$\gamma \otimes \beta=\gamma \beta$ & & $\begin{array}{l}\text { The attack accumulation coefficient is } \\
\text { obtained through the conjunction }{ }^{2} \text { of } \\
\text { the accuracy valuations corresponding } \\
\text { to the arguments that attack it. }\end{array}$ \\
\hline$\gamma \oplus \beta=\gamma+\beta-\gamma \beta$ & & $\begin{array}{l}\text { The strengthening operator combines } \\
\text { the accuracy associated with the infor- } \\
\text { mation provided by the argument and } \\
\text { the supporting valuation obtained for it, } \\
\text { with a penalty term representing an un- } \\
\text { certain level. }\end{array}$ \\
\hline$\gamma \ominus \beta=\left\{\begin{array}{l}\frac{\gamma-\beta}{1-\beta} \\
0\end{array}\right.$ & $\begin{array}{l}\text { if } \gamma \geq \beta, \beta \neq 1 \\
\text { otherwise. }\end{array}$ & $\begin{array}{l}\text { The weakening operation obtains the } \\
\text { valuation associated with an attacked } \\
\text { argument through a gradual degrada- } \\
\text { tion in case that the attackers are not } \\
\text { strong enough to defeat it; thus, if the } \\
\text { accumulation of the attackers' strength } \\
\text { is } T \text {, then the argument is wholly de- } \\
\text { feated. }\end{array}$ \\
\hline
\end{tabular}

The domain of labels $\mathfrak{L}_{2}$ of algebra $\mathrm{B}$ is the real interval $[0,1]$ representing a normalized reliability valuation with $T=1$ and $\perp=0$. The reliability valuation associated with an argument is related to the reliability of its source. The accumulation, strengthening, and weakening operators established to manipulate this feature are defined as follows:

\begin{tabular}{|l|l|}
\hline \multicolumn{2}{|c|}{ Reliability } \\
\hline$\gamma \odot \beta=\gamma \beta$ & $\begin{array}{l}\text { The support accumulation coefficient is } \\
\text { obtained through the conjunction of the } \\
\text { reliability valuations corresponding to } \\
\text { the arguments that support it. }\end{array}$ \\
\hline
\end{tabular}

2. The product t-norm is the standard semantics for strong conjunction in product fuzzy logic. 


\begin{tabular}{l|lllllllllll} 
& \multicolumn{1}{|c}{ Argument } \\
$v$ & $\mathrm{~A}$ & $\mathrm{~B}$ & $\mathrm{C}$ & $\mathrm{D}$ & $\mathrm{E}$ & $\mathrm{F}$ & $\mathrm{G}$ & $\mathrm{H}$ & $\mathrm{I}$ & $\mathrm{J}$ & $\mathrm{K}$ \\
\hline$\alpha_{1}$ & 0.6 & 0.2 & 0.3 & {$[0.6,0.8]$} & 0.4 & 0.6 & 0.6 & 0.8 & {$[0.5,0.6]$} & 0.7 & 0.7 \\
$\alpha_{2}$ & 0.6 & 0.5 & 0.2 & 0.8 & 0.2 & 0.8 & 0.6 & {$[0.5,0.7]$} & {$[0.7,0.9]$} & 0.6 & 0.7
\end{tabular}

Table 3: Initial argument valuations

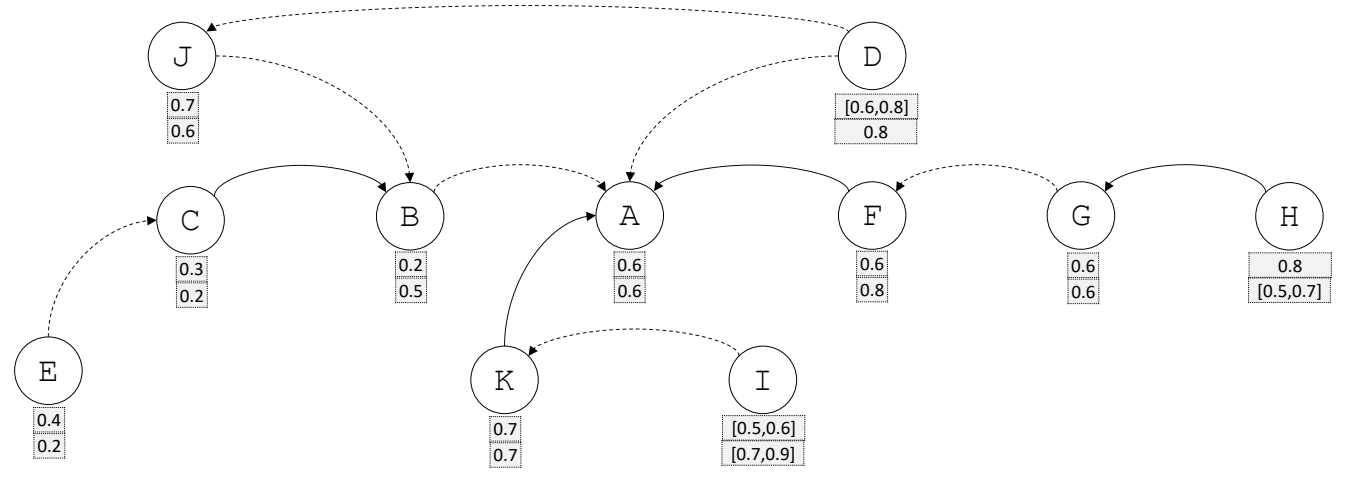

Figure 6: Initial labeled bipolar argumentation graph.

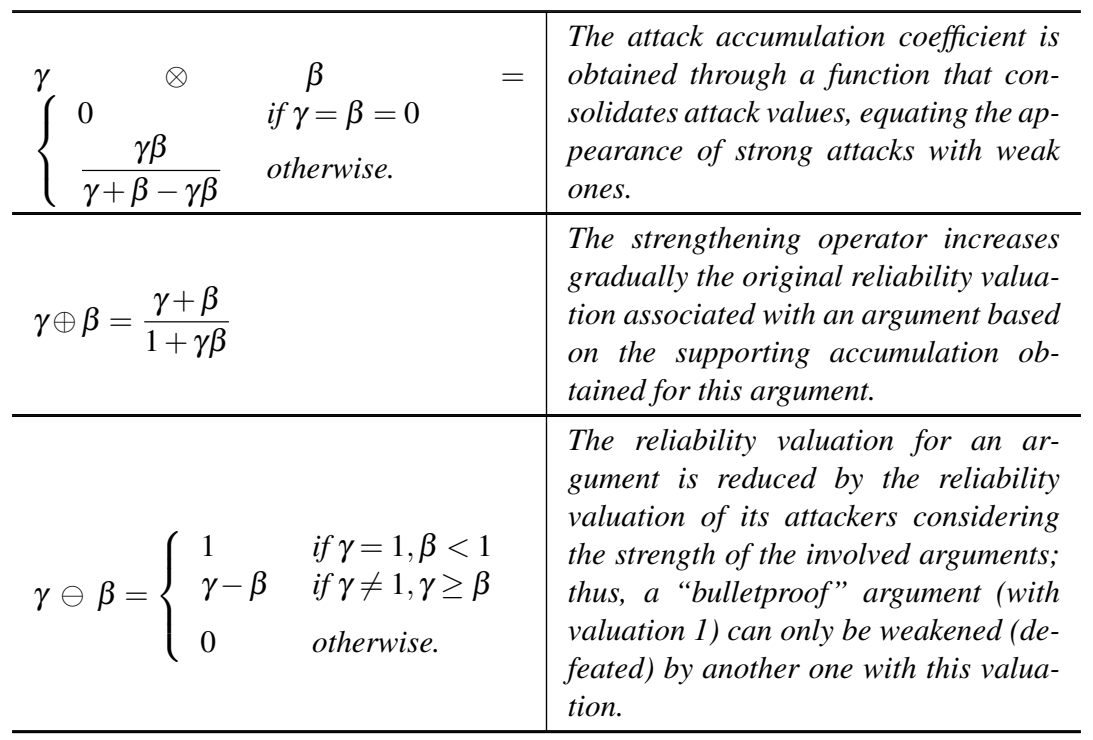

and $v(\mathrm{AR})$ determines the original valuation associated with each argument (see Table 3). We annotate the attribute of an argument with an interval $[x, y]$ to represent the variation of its attribute produced by the agent's perception uncertainty. In Table 3, $\alpha_{1}$ (resp. $\left.\alpha_{2}\right)$ corresponds to algebra A (resp. B).

Analyzing L-BAF $\Psi$ (cf. Figure 6) using the classical bipolar notions, we have the extensions obtained in the Example 1, where $S_{1}=\{\mathrm{I}, \mathrm{K}, \mathrm{E}, \mathrm{C}, \mathrm{H}\}$ is d-admissible, s-admissible, and c-admissible, while $S_{2}=\{\mathrm{I}, \mathrm{K}, \mathrm{E}, \mathrm{C}, \mathrm{D}, \mathrm{J}, \mathrm{H}\}$ is d-admissible, but not s-admissible and not c-admissible. Recall that 


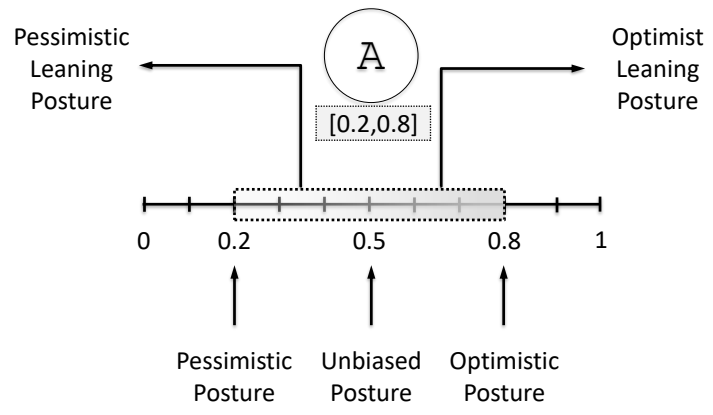

Figure 7: Example of different possible users' points of view.

the set $S_{2}$ is a d-preferred extension, since it is the maximal d-admissible set; however, in this case, there exists neither non-trivial s-preferred extension, nor non-trivial c-preferred extension.

The features associated with the arguments establish an argument classification giving an idea of how important they are in the argumentation process. Next, we present the treatment of argument features in the bipolar argumentation domain and how they affect the argument acceptability process.

\section{Labeling Procedure in $L A F$}

Once the argumentation graph is built, its corresponding solution space arises from the variation associated with each label - this variation appears due to a variety of causes, such as uncertainty associated with the agent's perception, preferences (with different degrees of tolerance), their optimistic or pessimistic inclinations, or inherent feature variation over time, among others. However, users may be interested in a particular subset of solutions where specific preferences are taken into account. Users can thus decide to take a posture (or stance) towards this variation depending on their interests; the purpose behind this process is to represent the users' point of view over a particular discussion, optimizing the features associated with a specific set of arguments with respect to such views ( $c f$. Figure 7).

From a computational point of view, the posture feature is a tool that allows to simplify ranges into single values, allowing us to collapse multiple options into one in cases in which the user does not wish to entertain different possibilities. For example, consider the case of a user who wishes to be completely conservative in their evaluation of uncertainty; here, lower bounds can always be taken instead of entire intervals, leading to a much smaller and simpler solution space. Thus, the user posture function affords significant reductions in the computational cost of the labeling process by reducing the space of possible models to a single one. By adopting a selection criterion for the users' posture (such as pessimistic, leaning pessimistic, unbiased, or other similar stances), the possible values are reduced from any value within an interval—which causes a combinatorial blowup when considering the entire set of arguments - to a single value representative of their posture. We also use this function as a way to simplify our examples and the presentation of intuitions in general. However, the use of a posture function can be considered an optional tool, choosing to work with the entire space of solutions accepting the computational costs derived from the selection of the operators to carry out the propagation of attribute value ranges. An analysis establishing a particular point of view is used extensively in many areas of decision-making, such as engineering design 
(Baroni et al., 2015), medical procedures (Fox et al., 2007), legal case analysis (Palmirani, 2018), and financial portfolio selection (Cruz-Reyes et al., 2014).

Definition 10 (Posture Function) Let $\Psi$ be an L-BAF. The posture function $\rho$ is defined as follows

$$
\rho: \mathrm{AR} \longrightarrow\left|\mathrm{A}_{1}\right| \times\left|\mathrm{A}_{2}\right| \times \ldots \times\left|\mathrm{A}_{n}\right|
$$

such that

$$
\rho(\mathrm{A})=\left(\rho_{\mathrm{A}}^{1}(v(\mathrm{~A})), \rho_{\mathrm{A}}^{2}(v(\mathrm{~A})), \ldots, \rho_{\mathrm{A}}^{n}(v(\mathrm{~A}))\right)
$$

where

$$
\rho_{\mathrm{A}}^{i}: \mathscr{S}([\perp, \top]) \rightarrow[\perp, \top], 1 \leq i \leq n,
$$

where $\mathscr{S}([\perp, \top])=\{[\alpha, \beta] \mid \perp \leq \alpha \leq \beta \leq \top\}$ and $\rho_{\mathrm{A}}^{i}$ is any function given by the user such that $\rho_{\mathrm{A}}^{i}([\alpha, \beta])=\alpha$ if $\alpha=\beta$, and $\rho_{\mathrm{A}}^{i}([\alpha, \beta])=\gamma$ with $\gamma \in[\alpha, \beta] .^{3}$

We can extend the posture function to select a specific posture for each attribute associated with each argument. However, in order to simplify our formalism we only consider a single posture for all the features associated with the arguments.

Example 3 Continuing with Example 2, suppose that we are interested in optimizing the argumentation discussion representing our posture over the uncertainty intrinsically associated with the information provided by the domain. In this sense, we can establish the posture over the arguments through the function $\rho$ as follows:

\begin{tabular}{l|l|l} 
Optimistic Posture & Pessimistic Posture & Unbiased Posture \\
$\rho_{\mathrm{D}}: \max ([\gamma, \beta])$ & $\rho_{\mathrm{I}}: \min ([\gamma, \beta])$ & $\rho_{\mathrm{H}}: \operatorname{med}([\gamma, \beta])$
\end{tabular}

In this case, we take an optimistic posture over argument $\mathrm{D}$, while a pessimistic posture is established for argument I; on the other hand, we consider an optimistic leaning (or unbiased) posture 4 over argument $\mathrm{H}$. For reasons of readability, we do not include the position functions that determine the valuations of the arguments with single values. Thus, the final attribute table associated with the arguments is the following:

\begin{tabular}{l|lllllllllll} 
& \multicolumn{10}{|c}{ Argument } \\
$v$ & A & B & C & D & E & F & G & H & I & J & K \\
\hline$\alpha_{1}$ & 0.6 & 0.2 & 0.3 & 0.8 & 0.4 & 0.6 & 0.6 & 0.8 & 0.5 & 0.7 & 0.7 \\
$\alpha_{2}$ & 0.6 & 0.5 & 0.2 & 0.8 & 0.2 & 0.8 & 0.6 & 0.6 & 0.7 & 0.6 & 0.7
\end{tabular}

Now, we proceed to attach a label to each argument (node), representing valuations referring to extra information that we want to represent. Next, we present the labeling procedure for an argumentation graph, which derives a system of equations that characterizes the knowledge behavior contained in the argumentation discussion.

3. The elements of $\mathscr{S}$ are sometimes referred to as segments.

4. The med operation corresponds to the median (the middle number of a finite sorted sequence.) 
Definition 11 (Labeling Procedure) Let $\Psi=\langle\Theta, \mathbf{A}, v\rangle$ be an $L-B A F$ where $\Theta$ is the underlying $B A F, \mathrm{G}_{\Theta}$ be the corresponding argumentation graph for $\Theta,{ }^{5}$ and $\mathrm{A}$ be an algebra in $\mathbf{A}$. The labeling of an argument $\mathrm{A}$ is the assignment of three valuations from each of the algebras defined in $\Theta$, denoted with $\left\langle\alpha^{\mathrm{A}}, \mu^{\mathrm{A}}, \delta^{\mathrm{A}}\right\rangle$, where $\alpha^{\mathrm{A}}$ is the original value of the attribute assigned to the argument by $\rho$ that considers a user's posture, $\mu^{\mathrm{A}}$ is the strengthened valuation that represents the accumulation of the attributes of arguments supporting $\mathrm{A}$, and $\delta^{\mathrm{A}}$ is the overall valuation obtained after taking the attacks and supports into consideration. If $\mathrm{A}$ is an argument defined in $\Theta$, its valuations $\left\langle\alpha^{\mathrm{A}}, \mu^{\mathrm{A}}, \delta^{\mathrm{A}}\right\rangle$ with respect to $\mathrm{A}$ are determined as follows:

$-\alpha^{\mathrm{A}}=\rho(\mathrm{A})$.

$-\operatorname{If}(\overrightarrow{\mathrm{A}})=\emptyset$, then $\mu^{\mathrm{A}}=\alpha^{\mathrm{A}}$.

- If $\overrightarrow{(\mathrm{A})} \neq \emptyset$, then $\mu^{\mathrm{A}}=\alpha^{\mathrm{A}} \oplus\left(\odot_{j=1}^{m} \delta^{\mathrm{x}_{j}}\right)$, with $\mathrm{x}_{j} \in(\overrightarrow{\mathrm{A}})$.

- If $\overrightarrow{(\mathrm{A})}=\emptyset$, then $\delta^{\mathrm{A}}=\mu^{\mathrm{A}}$.

- If $\overrightarrow{(\mathrm{A})} \neq \emptyset$ and $\overrightarrow{(\mathrm{A})}=\emptyset$, then $\delta^{\mathrm{A}}=\alpha^{\mathrm{A}} \ominus\left(\otimes_{k=1}^{n} \delta^{\mathrm{x}_{k}}\right)$ with $\mathrm{X}_{k} \in \overrightarrow{(\mathrm{A})}$.

- If $\overrightarrow{(\mathrm{A})} \neq \emptyset$ and $\overrightarrow{(\mathrm{A})} \neq \emptyset$, then

$$
\delta^{\mathrm{A}}= \begin{cases}\alpha^{\mathrm{A}} \oplus\left(\left(\odot_{j=1}^{m} \delta^{\mathrm{X}_{j}}\right) \ominus\left(\otimes_{k=1}^{n} \delta^{\mathrm{Y}_{k}}\right)\right) & \text { when } \otimes_{k=1}^{n} \delta^{\mathrm{Y}_{k}} \leq \odot_{j=1}^{m} \delta^{\mathrm{x}_{j}} \\ & \text { with } \mathrm{Y}_{k} \in \overrightarrow{(\mathrm{A})} \text { and } \mathrm{X}_{j} \in(\mathrm{A}) \\ \alpha^{\mathrm{A}} \ominus\left(\left(\otimes_{k=1}^{n} \delta^{\mathrm{Y}_{k}}\right) \ominus\left(\odot_{j=1}^{m} \delta^{\mathrm{x}_{j}}\right)\right) & \text { when } \otimes_{k=1}^{n} \delta^{\mathrm{Y}_{k}}>\odot_{j=1}^{m} \delta^{\mathrm{x}_{j}} \\ & \text { with } \mathrm{Y}_{k} \in \overrightarrow{(\mathrm{A})} \text { and } \mathrm{X}_{j} \in(\mathrm{A})\end{cases}
$$

The labeling is completed when all arguments defined in $\Theta$ are assigned a label. We use the term labeled bipolar graph to refer to a complete labeling associated with the bipolar argumentation graph.

Thus, the labeling of arguments begins with the function $\rho$, obtaining the original valuation for each attribute representing the user's posture. It then propagates from the leaves ${ }^{6}$, where the original valuation coincides with the strengthened and overall valuations, to the rest of the nodes. The strengthened valuation (the original valuation reinforced by the accumulation of valuations of its supporting arguments) is determined for a given node. In the final step, the overall valuation is obtained, where the support accumulation of valuations can face the accumulated values of the attacking arguments and then the residual strength of this confrontation is applied over the original valuation. For each $A \in A R$ and for each algebra $A \in A$, representing a feature to be associated with $\mathrm{A}$, the triple $\left\langle\alpha^{\mathrm{A}}, \mu^{\mathrm{A}}, \delta^{\mathrm{A}}\right\rangle$ is called the label of A with respect to $\mathrm{A}$.

In the $B A F$ proposed by (Cayrol \& Lagasquie-Schiex, 2005b), the support relation is assumed to be independent of the attack relation modeling a positive relation between arguments, while attacks

5. We refer to the classical bipolar argumentation framework associated with $\Theta$.

6. We will say that a node (or argument) is a leaf if and only if there do not exist incoming arcs of any type. That is, an argument is a leaf if and only if there are no supporting or attacking argument that affect it. 


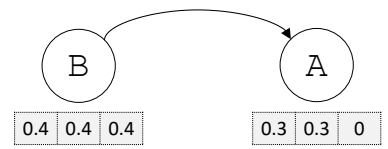

(a)

Defeat effect from B to A

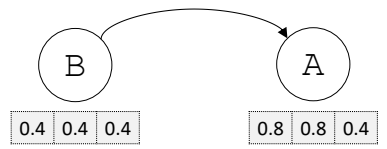

(b)

Weakening effect from $B$ to $A$

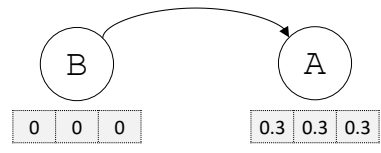

(c)

Non-effect from B to A

Figure 8: Example of attack effects in the $L-B A F \Psi$.

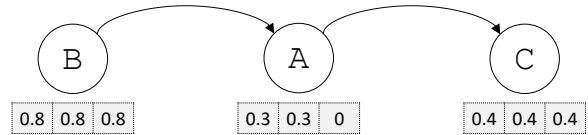

(a)

Total defense relation from $\mathrm{B}$ to $\mathrm{C}$ of A attack's

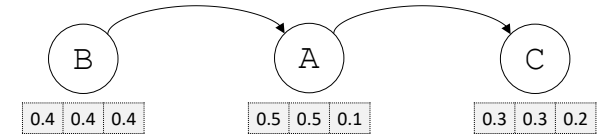

(b)

Partial defense relation from $B$ to $C$ of A attack's

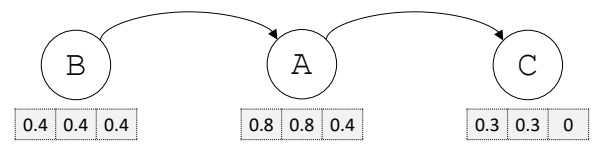

(c)

Fail defense relation from $\mathrm{B}$ to $\mathrm{C}$ of A attack's

Figure 9: Example of defense in the $L-B A F \Psi$ through defender arguments.

are considered as defeats signifying a negative intention. However, unlike the original $B A F$, an attack does not always imply a defeat in our proposal. Based on the weakening operator defined in the algebra of argumentation labels, attacks between arguments can weaken the valuations associated with the attacked argument, resulting in a defeat or a weakening. In contrast, no effect over the valuations associated with the attacked argument is produced when all the attacking arguments have the least possible feature degree (cf. Figure 8). So, when an argument experiences a weakening effect, it can be understood as the result of defending itself from an attacker. In the figures that follow, the label attached to each argument $\mathrm{A} \in \mathrm{AR}$ contains the three values $\alpha^{\mathrm{A}}, \mu^{\mathrm{A}}, \delta^{\mathrm{A}}$ obtained through the labeling process; that triplet can be seen as a row in a $1 \times 3$ matrix. So, if an argument A $\in$ AR has $n$ features, then its valuations are identified by an $n \times 3$ matrix (see Figure 12).

The notion of defense is implicit in the propagation of the arguments' valuations. For example, an argument $\mathrm{C}$ that defends an argument A from its attacker B can lead to different situations: the defense argument is good enough to neutralize the attack (cf. Figure 9 (a)); the defense argument allows to decrease the force of the attack, possibly producing a weakening in the attacked argument (cf. Figure 9 (b)); or defeating it, case in which the defense is not effective (cf. Figure 9 (c)).

Furthermore, support can be interpreted in our formalism as a defense relation that neutralizes or weakens the attacker's strength. On the one hand, the accumulation of the strength that originated from the supporting arguments may not be sufficient to neutralize the accumulation of the attacker's strength. Also, the residual strength of this confrontation may be sufficient to defeat or weaken the argument under attack (cf. Figure 10 (a) and Figure 10 (b), respectively). On the other hand, the strength of the supports may neutralize the strength of attacks effectively; so, the attacked argument would be wholly defended or even strengthened (cf. Figure 10 (c)). 


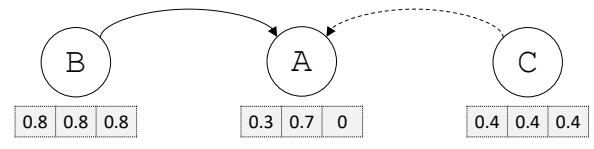

(a)

Fail defense relation from $\mathrm{C}$ to $\mathrm{A}$

of B attack's

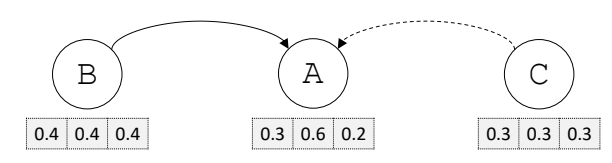

(b)

Partial defense relation from $\mathrm{C}$ to $\mathrm{A}$ of B attack's

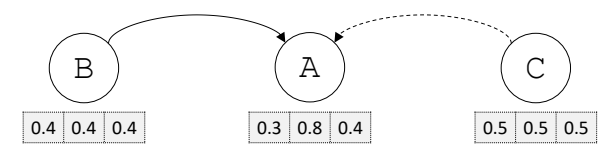

(c)

Total defense relation from $\mathrm{C}$ to $\mathrm{A}$ of B attack's

Figure 10: Example of defense in the $L-B A F \Psi$ through supporting arguments.

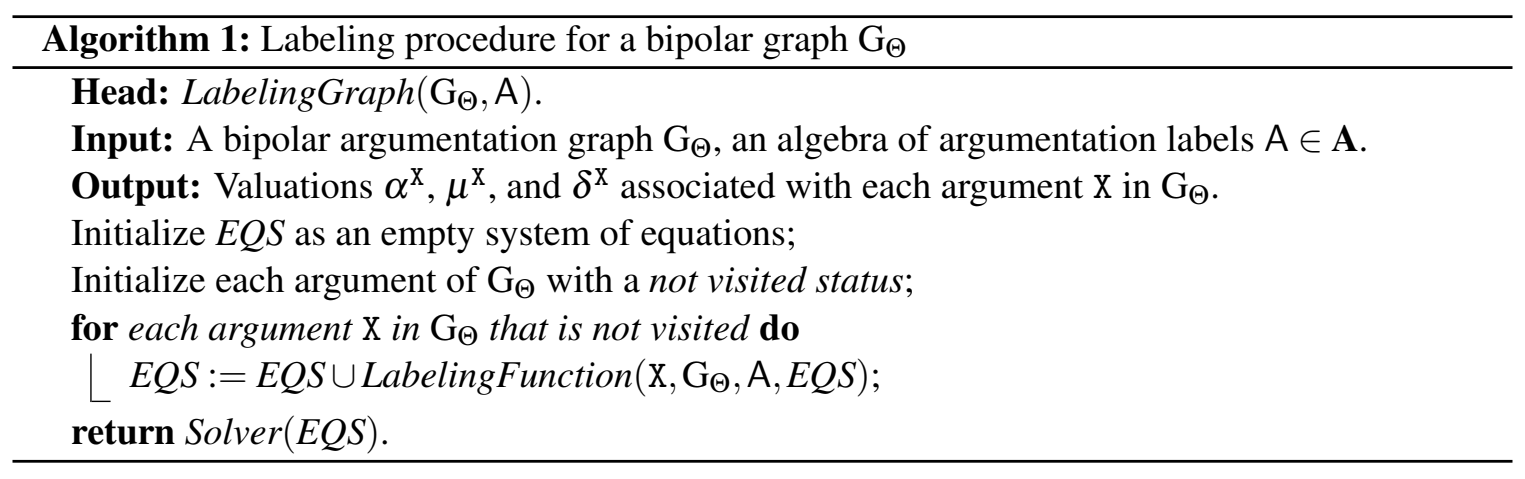

In what follows, we present the algorithms for obtaining the valuations associated with each argument in the bipolar argumentation graph. Algorithm 1, using Algorithm 2, produces a system of equations to be solved by using a solver-the specific solver used will depend on how the operators are defined in the algebra. Algorithm 2 analyzes each node of the graph with the purpose of specifying the equations that determine the valuations of its arguments analyzing the relations between them. To do this, it first analyzes the supporting arguments associated with an argument to determine the support valuation associated with it; then, it specifies the equation that determines the overall valuation of the input argument A based on the overall valuation associated with the attacking arguments.

It is significant to mention that the propagation of the characteristics through a support or attack chain is based on the overall valuation associated with each argument that comprises such chain, giving rise to dependencies in the propagation through the argumentation graph.

The following result states the computational cost of this procedure.

Proposition 1 The worst-case running time of the labeling procedure for the bipolar argumentation graph $\mathrm{G}_{\Theta}$ is $O(n \times(m+t))$, where $n$ is the cardinality associated with the set of arguments AR, $m$ is the maximal number of arguments with an attacking role for an argument $\mathrm{X} \in \mathrm{AR}$, and $t$ is the maximal number of arguments with a supporting role for an argument $\mathrm{X} \in \mathrm{AR}$. 


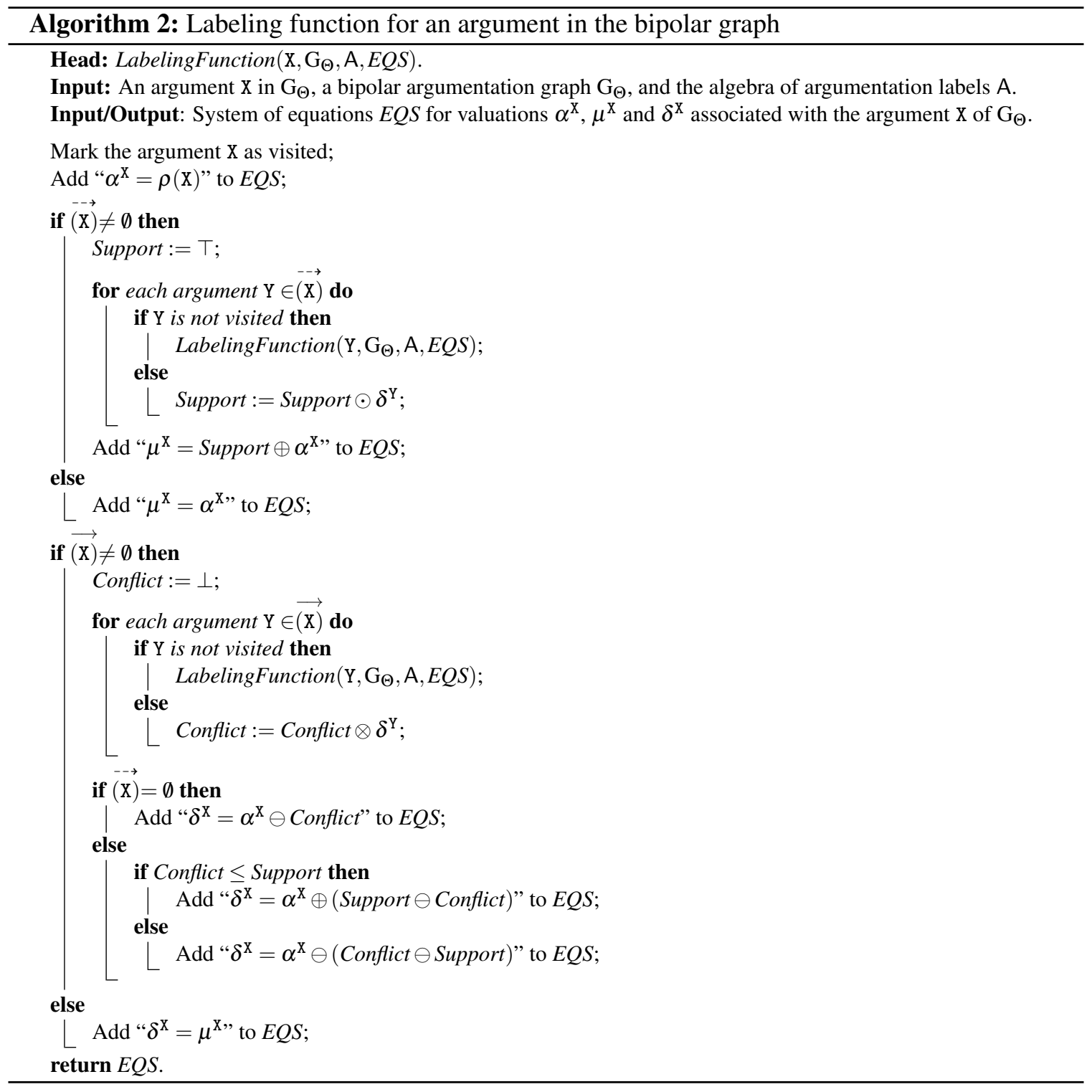


The semantics of a bipolar argumentation framework is determined by the possible solutions to the system of equations that is generated by the labeling process-each solution is a valid labeling for the argumentation graph. Formally, we have:

Definition 12 (Valid Labeling for an $L-B A F$ ) Let $\Psi=\langle\Theta, \mathbf{A}, v\rangle$ be an $L-B A F$ where $\Theta$ is the underlying $B A F, \mathrm{G}_{\Theta}$ be the corresponding argumentation graph for $\Theta$, and EQS be the corresponding system of equations representing the constraints that all the valuations associated with the arguments of $\mathrm{G}_{\Theta}$ must satisfy. A valid labeling for $\mathrm{G}_{\Theta}$, denoted $\lambda(\Theta)$, is any set of values $\alpha^{\mathrm{x}}, \mu^{\mathrm{x}}$, and $\delta^{\mathrm{X}}, \forall \mathrm{X} \in \mathrm{G}_{\Theta}$, that constitutes a solution for EQS. We denote with $\Lambda_{\Psi}$ the set of all the valid labelings for $\Psi$, and denote with $\mathrm{G} \Psi$ the labeled bipolar graph for $\Psi$.

Since the labeling process incorporates the user's posture, the system's solution, and therefore the labeled graph, is unique. The following theorem formalizes that result.

Theorem 1 Let $\Psi=\langle\Theta, \mathbf{A}, v\rangle$ be an $L-B A F$ where $\Theta$ is the underlying BAF, $\mathrm{G}_{\Theta}$ be the corresponding argumentation graph for $\Theta$, and $\lambda_{1}(\Theta)$ and $\lambda_{2}(\Theta)$ be two different labeling sequences for $\mathrm{G}_{\Theta}$, where $\lambda_{1}(\Theta)$ generates system $E Q S_{1}$ and $\lambda_{2}(\Theta)$ generates system $E Q S_{2}$. Then, we have that $E Q S_{1}=E Q S_{2}$.

Another interesting result is that the valuations associated with the arguments are always in the desired range.

Lemma 1 Let $\Psi=\langle\Theta, \mathbf{A}, v\rangle$ be an $L-B A F$ where $\Theta$ is the underlying $B A F, \mathrm{G}_{\Psi}$ be the associated labeled bipolar graph, and $\mathrm{X}$ be an argument in $\mathrm{G}_{\Psi}$. Then, for every algebra $\mathrm{A} \in \mathbf{A}$, the labels $\left\langle\alpha^{\mathrm{X}}\right.$, $\left.\mu^{\mathrm{X}}, \delta^{\mathrm{X}}\right\rangle$ related to $\mathrm{A}$ satisfy: $(i) \alpha^{\mathrm{X}} \in[\perp, \top],(i i) \mu^{\mathrm{X}} \in\left[\alpha^{\mathrm{X}}, \top\right]$, and (iii) $\delta^{\mathrm{X}} \in\left[\perp, \mu^{\mathrm{X}}\right]$.

Furthermore, the original, strengthened, and overall valuations of an argument are closely related; the valuations associated with each argument in the graph corresponding to a valid labeling satisfy the following properties.

Lemma 2 Let $\Psi=\langle\Theta, \mathbf{A}, v\rangle$ be an $L-B A F$ where $\Theta$ is the underlying $B A F, \Lambda_{\Psi}$ be the set of all the valid labelings for $\Psi, \mathrm{G}_{\Psi}$ be the associated labeled bipolar graph, and $\mathrm{X}$ be an argument in $\mathrm{G}_{\Psi}$. Then, for every algebra $\mathrm{A} \in \mathbf{A}$, the labels $\left\langle\alpha^{\mathrm{X}}, \mu^{\mathrm{x}}, \delta^{\mathrm{x}}\right\rangle$ related to $\mathrm{A}$ satisfy:

(i) $\mu^{\mathrm{X}} \geq \delta^{\mathrm{x}}$;

(ii) $\mu^{\mathrm{X}} \geq \alpha^{\mathrm{X}}$; and

(iii) If $\mu^{\mathrm{x}}=\perp$, then $\delta^{\mathrm{x}}=\alpha^{\mathrm{x}}=\perp$.

As a consequence of the previous results, it is possible to assert that the labeling process produces a set of valid labelings that is sound and complete. That is to say that if there exists a valid labeling for a bipolar argumentation graph $\mathrm{G}_{\Theta}$, that labeling belongs to $\Lambda_{\Psi}$; moreover, all the labelings that belong to $\Lambda_{\Psi}$ are adequate solutions for $\mathrm{G}_{\Theta}$. Formally:

7. We refer to the classical bipolar argumentation framework where each argument is labeled with the original, strengthened, and overall valuations. 


$$
\left\{\begin{array}{l}
e_{1}^{\mathrm{X}}: \mu_{\mathrm{X}}^{\mathrm{K}}=\alpha_{\mathrm{X}}^{\mathrm{K}} \oplus \delta_{\mathrm{X}}^{\mathrm{I}} \\
e_{2}^{\mathrm{X}}: \delta_{\mathrm{X}}^{\mathrm{K}}=\mu_{\mathrm{X}}^{\mathrm{K}} \\
\mathrm{e}_{3}^{\mathrm{X}}: \mu_{\mathrm{X}}^{\mathrm{C}}=\alpha_{\mathrm{X}}^{\mathrm{C}} \oplus \delta_{\mathrm{X}}^{\mathrm{E}} \\
\mathrm{e}_{4}^{\mathrm{X}}: \delta_{\mathrm{X}}^{\mathrm{C}}=\mu_{\mathrm{X}}^{\mathrm{C}} \\
\mathrm{e}_{5}^{\mathrm{X}}: \mu_{\mathrm{X}}^{\mathrm{J}}=\alpha_{\mathrm{X}}^{\mathrm{J}} \oplus \delta_{\mathrm{X}}^{\mathrm{D}} \\
\mathrm{e}_{6}^{\mathrm{X}}: \delta_{\mathrm{X}}^{\mathrm{J}}=\mu_{\mathrm{X}}^{\mathrm{J}} \\
\mathrm{e}_{7}^{\mathrm{X}}: \mu_{\mathrm{X}}^{\mathrm{B}}=\alpha_{\mathrm{X}}^{\mathrm{B}} \oplus \delta_{\mathrm{X}}^{\mathrm{J}} \\
e_{\mathrm{X}}^{\mathrm{X}}: \delta_{\mathrm{X}}^{\mathrm{B}}=\alpha_{\mathrm{X}}^{\mathrm{B}} \oplus\left(\delta_{\mathrm{X}}^{\mathrm{J}} \ominus \delta_{\mathrm{X}}^{\mathrm{C}}\right) \\
e_{9}^{\mathrm{X}}: \mu_{\mathrm{X}}^{\mathrm{G}}=\alpha_{\mathrm{X}}^{\mathrm{G}} \\
\mathrm{e}_{10}^{\mathrm{X}}: \delta_{\mathrm{X}}^{\mathrm{G}}=\alpha_{\mathrm{X}}^{\mathrm{G}} \ominus \delta_{\mathrm{X}}^{\mathrm{H}} \\
\mathrm{e}_{11}^{\mathrm{X}}: \mu_{\mathrm{X}}^{\mathrm{F}}=\alpha_{\mathrm{X}}^{\mathrm{F}} \oplus \delta_{\mathrm{X}}^{\mathrm{G}} \\
e_{12}^{\mathrm{X}}: \delta_{\mathrm{X}}^{\mathrm{F}}=\mu_{\mathrm{X}}^{\mathrm{F}} \\
e_{13}^{\mathrm{X}}: \mu_{\mathrm{X}}^{\mathrm{A}}=\alpha_{\mathrm{X}}^{\mathrm{A}} \oplus\left(\delta_{\mathrm{X}}^{\mathrm{B}} \odot \delta_{\mathrm{X}}^{\mathrm{D}}\right) \\
\mathrm{e}_{14}^{\mathrm{X}}: \delta_{\mathrm{X}}^{\mathrm{A}}=\alpha_{\mathrm{X}}^{\mathrm{A}} \ominus\left(\left(\delta_{\mathrm{X}}^{\mathrm{K}} \otimes \delta_{\mathrm{X}}^{\mathrm{F}}\right) \ominus\left(\delta_{\mathrm{X}}^{\mathrm{B}} \odot \delta_{\mathrm{X}}^{\mathrm{D}}\right)\right)
\end{array}\right\}
$$

Figure 11: System of equations for Example 1, $X \in \mathbf{A}=\{A, B\}$.

Theorem 2 Let $\Psi=\langle\Theta, \mathbf{A}, v\rangle$ be an $L-B A F$ where $\Theta$ is the underlying $B A F, \mathrm{G}_{\Theta}$ be the corresponding argumentation graph for $\Theta$, and $\Lambda_{\Psi}$ be the set of all the valid labelings for $\Psi$. Then, $\Lambda_{\Psi}$ is sound and complete.

Example 4 Continuing Example 3, we now turn to our approach (cf. Figure 12) in order to analyze the relation between the arguments. We propagate the valuations associated with each argument following the procedure from Definition 11; through this process, we obtain the system of equations EQS (cf. Figure 11) for the argumentation graph $\mathrm{G}_{\Theta}$. For reasons of readability, we do not include the equations that determine the valuations of the leaf nodes, which are trivial.

Let us observe that the labeled argumentation graph captures the argumentation behavior in a specific domain, where the features associated with the arguments reflect how these entities are related; this is captured by the system EQS. For this example, two sets of equations are obtained, one for each algebra in $\mathbf{A}=\{\mathrm{A}, \mathrm{B}\}$. At first glance, the equations appear to be the same, but they produce different expressions when the operators defined in the corresponding algebra are instantiated. For instance, considering the first equation in Figure 11:

$$
\mathrm{e}_{1}^{\mathrm{X}}: \mu_{\mathrm{X}}^{\mathrm{K}}=\alpha_{\mathrm{X}}^{\mathrm{K}} \oplus \delta_{\mathrm{X}}^{\mathrm{I}}
$$

when instantiated using algebra $\mathrm{A}$ renders:

$$
\mathrm{e}_{1}^{\mathrm{A}}: \mu_{\mathrm{A}}^{\mathrm{K}}=\alpha_{\mathrm{A}}^{\mathrm{K}}+\delta_{\mathrm{A}}^{\mathrm{I}}-\alpha_{\mathrm{A}}^{\mathrm{K}} \delta_{\mathrm{A}}^{\mathrm{I}}
$$

but when instantiated using algebra $\mathrm{B}$ results in:

$$
\mathrm{e}_{1}^{\mathrm{B}}: \mu_{\mathrm{B}}^{\mathrm{K}}=\frac{\alpha_{\mathrm{B}}^{\mathrm{K}}+\delta_{\mathrm{B}}^{\mathrm{I}}-2 \alpha_{\mathrm{B}}^{\mathrm{K}} \delta_{\mathrm{B}}^{\mathrm{I}}}{1-\alpha_{\mathrm{B}}^{\mathrm{K}} \delta_{\mathrm{B}}^{\mathrm{I}}}
$$

Briefly summarizing, the underlying principles below can be directly mapped to the principles laid out by (Cayrol \& Lagasquie-Schiex, 2005a) and Bonzon et al. (2016), and are satisfied by all 


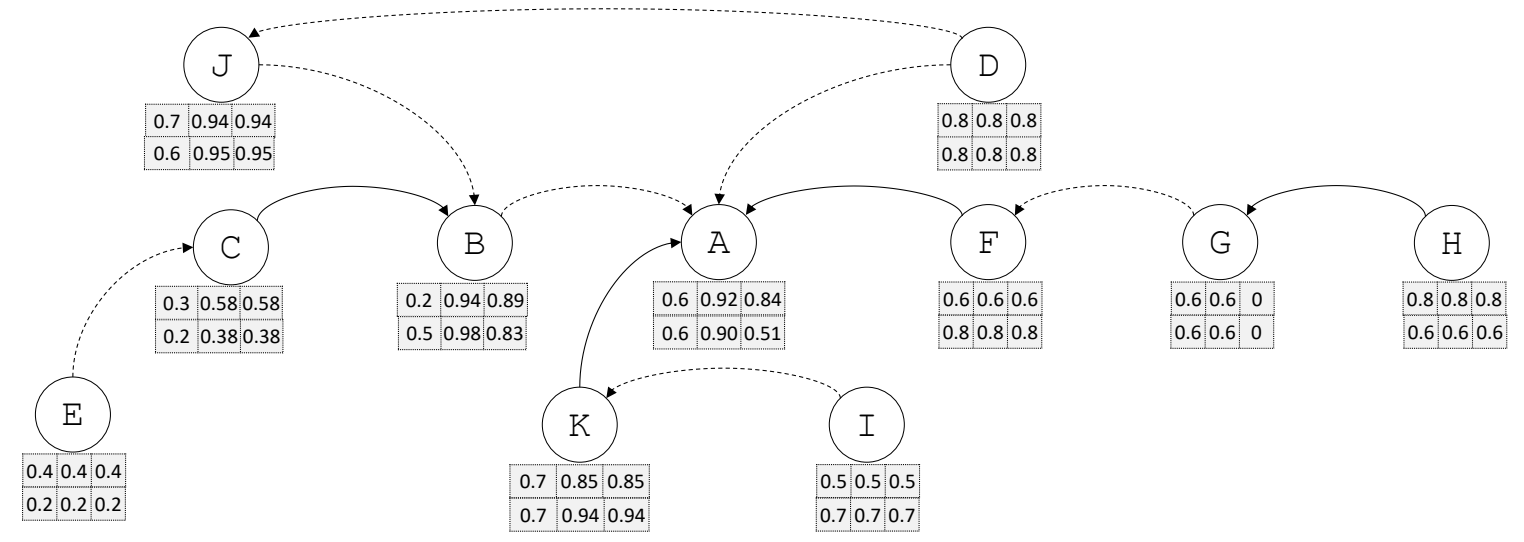

Figure 12: Example for labeled bipolar argumentation graph $\mathrm{G}_{\Psi}$.

the valuations defined according to our labeling process. In general, these principles describe the behavior of valuations associated with arguments in our framework.

Principle 1 The system of equations described by Definition 11 and the valid labelings that satisfy such constraints (Definition 12) enjoy the following principles:

- Total Order: All pairs of arguments can be compared.

- Argument Equivalence: If there exists a graph isomorphism between the ancestors' graph $^{8}$ of two arguments, then they are equally acceptable.

- Ordinal Equivalence: If two arguments A and B have the same number of direct attackers and supporters, and for each direct attacker of A there exists a direct attacker of B such that the two attackers are equally acceptable, then $\mathrm{A}$ and $\mathrm{B}$ are equally acceptable too.

- Attack Strength: The overall valuation for an argument depends, in a non-increasing manner, on the overall valuation of the attacking argument.

- Support Strength: The overall valuations of the supporting arguments contribute to increase the strengthened valuation of the supported argument.

- The Overall and Strengthened Valuations: The overall valuation is equal to the strengthened valuation for arguments without attackers; for an attacked but undefeated argument, the overall valuation is less than the strengthened valuation, whenever the attacking arguments are strong enough to weaken it.

Once the arguments are labeled, we can consider their acceptability status; to accomplish this, we will use the information attached to the arguments to provide different degrees, or rankings, of acceptability. We will explore this issue in the following section.

8. The predecessor of an argument together with all the ancestors of the predecessor of an argument. 


\section{Acceptability Process}

The problem of understanding the process of argumentation and its role in human reasoning has been studied by many researchers in different fields, including philosophy, logic, and AI (Barth \& Krabbe, 2010; Toulmin, 2003). Briefly speaking, the idea of argumentation-based reasoning is that a claim is believable if it can be defended successfully against attacking arguments. In other words, whether or not a rational agent believes in a specific claim depends on whether or not the argument supporting this claim can be successfully defended against counterarguments.

Next, based on the arguments' values, we characterize the statuses associated with each one. The following classification is useful for representing the effect that the discussion has on each argument; the status is thus valuable as an illustration of the original argument's relationship with its "processed" version.

Definition 13 (Gradual Status Assignment in $\mathbf{L}-\boldsymbol{B A F}$ ) Let $\Psi=\langle\Theta, \mathbf{A}, v\rangle$ be an $L-B A F, G_{\Psi}$ be the corresponding labeled bipolar argumentation graph, and $\mathrm{A}$ be an argument (node) in $G \Psi$. For each algebra $\mathrm{A} \in \mathbf{A}$, the argument $\mathrm{A}$ is assigned one of four possible statuses obtained from the valuation $\left\langle\alpha^{\mathrm{A}}, \mu^{\mathrm{A}}, \delta^{\mathrm{A}}\right\rangle$ related to $\mathrm{A}$ as follows:

- Neutralized iff $\delta^{\mathrm{A}}=\perp$.

- Weakened iff $\perp<\delta^{\mathrm{A}}<\alpha^{\mathrm{A}}$.

- Strengthened iff $\alpha^{\mathrm{A}}<\delta^{\mathrm{A}}$.

- Unchallenged iff $\alpha^{\mathrm{A}}=\delta^{\mathrm{A}} \neq \perp$.

Finally, every argument is associated with a vector of values where each value is associated with a feature; each of these values reflects the argument's degree of acceptability regarding a particular feature. We denote with $\chi_{g}$ the gradual status assignment to the bipolar argumentation graph $G_{\Psi}$.

The goal is to obtain an acceptability status for the argument from that vector. One possibility is to assign the least value in the vector as the overall acceptability degree for the argument. It is interesting to observe that the decision to take the least value among all elements in the vector is just one possible option that represents the most conservative option overall; the mechanism can be generalized to include preferences over attributes (a similar approach is taken by (Kaci \& van der Torre, 2008)) or richer schemes that would allow for incorporating more complex choices. The analysis of these alternative schemas that offer many design decisions brought about at the moment of concrete implementation is outside the scope of this paper, but is part of future work to develop the formalism further. The characterization presented above is useful to represent how strong the supports and attacks are for a particular argument. For instance, strong arguments may be slightly weakened, but this categorization gives us the possibility to recognize that an attacked argument is not supported or, if it is supported, it has not enough strength to neutralize the attacks. It can be understood as a preprocessing to take advantage of the available extra information, which can be refined as discussed below.

Lemma 3 Let $\Psi=\langle\Theta, \mathbf{A}, v\rangle$ be an $L-B A F$, and $G_{\Psi}$ be the corresponding labeled bipolar argumentation graph. The gradual status assignment $\chi_{g}$ to $G_{\Psi}$ is unique. 
Based on the status assigned to each argument, it is possible to identify in AR four categories of arguments: neutralized, denoted as $\mathrm{AR}_{n}$; weakened, denoted as $\mathrm{AR}_{w}$; strengthened, denoted as $\mathrm{AR}_{s}$; and unchallenged, denoted as $\mathrm{AR}_{u}$. This categorization of AR can be seen as a finite cover of AR; that is, all arguments that belong to AR are categorized.

Proposition 2 Let $\Psi=\langle\Theta, \mathbf{A}, v\rangle$ be an $L-B A F, G_{\Psi}$ be the corresponding labeled bipolar argumentation graph, $\chi_{g}$ be the gradual status assignment to the bipolar graph $G_{\Psi}$, and $\mathrm{AR}_{n}, \mathrm{AR}_{w}, \mathrm{AR}_{s}$, and $\mathrm{AR}_{u}$ be a disjoint set of neutralized, weakened, strengthened, and unchallenged arguments in $\Psi$. Then, the collection of subsets $\left\{\mathrm{AR}_{n}, \mathrm{AR}_{w}, \mathrm{AR}_{s}, \mathrm{AR}_{u}\right\}$ is a finite cover of $\mathrm{AR}$.

Let us observe that, $\mathrm{AR}_{n}, \mathrm{AR}_{w}, \mathrm{AR}_{s}$, and $\mathrm{AR}_{u}$ cannot all be empty sets unless $\mathrm{AR}$ is empty, which represents a non-interesting case; hence, the finite cover of $A R$ can be seen as a partition of $A R$, leading to a set of properties over AR that we will further discuss below.

We generally wish to determine a consistent set of arguments in favor or against certain conclusions, commonly referred to as the semantics of acceptability. In this work, we will use the status associated with the arguments to define our semantics; clearly, these sets may not always be conflictfree or safe. Here, we use a preference relation, denoted with $\succ$, defined over AR that uses the extra information contained in the labels in order to obtain the different conflict-free subsets of arguments corresponding to each particular set of arguments. Defining preference between arguments is a complex task since in some cases their valuations combine more than one attribute. In the literature, different ways of addressing the issue of preference have been proposed (Domshlak et al., 2011, Kaci, 2011, Rossi et al., 2011, 2012). Among them, in argumentation we can mention a few: defining a syntactic approach based on the elements involved in the construction of arguments (Simari \& Loui, 1992), applying the minimax principle (Yager, 1983), establishing a discussion to determine the preference between arguments in a dynamic sense (Prakken \& Sartor, 1997), Labeled Argumentation Frameworks (Budán et al., 2015), and developing different measures between semantics examining their possible maximization or combination (Doutre \& Mailly, 2017) (a combination of the Argument Interchange Format with an Algebra of Argumentation Labels). Consequently, it is the user at the moment of implementation who must establish an adequate comparison criterion based on the domain of application.

Definition 14 Let $\Psi=\langle\Theta, A, v\rangle$ be an $L-B A F, \succ$ be a preference relation over $\mathrm{AR}$, and $S \subseteq \mathrm{AR}$ be a set of arguments. Then, $(i) S$ is conflict-free iff $\nexists \mathrm{A}, \mathrm{B} \in S$ such that $(\mathrm{B}, \mathrm{A}) \in \mathcal{R}_{a}$ with $\mathrm{B} \succ \mathrm{A}$; (ii) $S$ is safe iff $\nexists \mathrm{A} \in \mathrm{AR}$ and $\nexists \mathrm{B}, \mathrm{C} \in S$ such that $(\mathrm{B}, \mathrm{A}) \in \mathcal{R}_{a}$ with $\mathrm{B} \succ \mathrm{A}$ and either $\mathrm{C}$ supports $\mathrm{A}$ (thus A $\notin S)$, or $\mathrm{A} \in S$.

Only the direct support and attack relations will be considered since the argument features are propagated through the argumentation graph. In this way, the attack and support sequences are analyzed, and the final label associated with each argument that reflects the interaction between arguments is obtained. Then, we analyze only the direct relations, while considering a preference function to determine when an argument is better than another.

Next, we present the connection between a safe and a conflict-free set of arguments; this was introduced by (Cayrol \& Lagasquie-Schiex, 2005b) and, since our notion of safety and conflictfreeness are different, we will demonstrate that the same result holds. Then, considering the notions of conflict-freeness and safety defined in Definition 14, we have: 
Proposition 3 Let $\Psi=\langle\Theta, A, v\rangle$ be an $L-B A F, \succ$ be a preference relation over $\mathrm{AR}$, and $S \subseteq \mathrm{AR}$ be a set of arguments. Then:

(i) If $S$ is safe, then $S$ is conflict-free.

(ii) If $S$ is conflict-free and closed under support, then $S$ is safe.

Based on the previous notions, we establish the different acceptability extensions that combine the conflict-freeness and safety definitions (that identify internal and external coherence) with the status classification (that represents the final quality associated with the arguments after considering the relationships between them).

Definition 15 Let $\Psi=\langle\Theta, A, v\rangle$ be an $L-B A F$, and $\mathrm{AR}_{w}, \mathrm{AR}_{s}$, and $\mathrm{AR}_{u}$ be the sets of weakened, strengthened, and unchallenged arguments associated with $\Psi$. Then:

- $S \subseteq \mathrm{AR}_{w}$ is a $\mathrm{CF}-\mathrm{W}$ extension of $\Phi$ iff $S$ is a maximal conflict-free set;

- $S \subseteq \mathrm{AR}_{w}$ is an $\mathrm{S}-\mathrm{W}$ extension of $\Phi$ iff $S$ is a maximal safe set;

- $S \subseteq \mathrm{AR}_{w}$ is a $\mathrm{C}-\mathrm{W}$ extension of $\Phi$ iff $S$ is a maximal conflict-free set closed under $\mathcal{R}_{s}$;

- $S \subseteq \mathrm{AR}_{u}$ is a CF-U extension of $\Phi$ iff $S$ is a maximal conflict-free set;

- $S \subseteq \mathrm{AR}_{u}$ is an $\mathrm{S}-\mathrm{U}$ extension of $\Phi$ iff $S$ is a maximal safe set;

- $S \subseteq \mathrm{AR}_{u}$ is a $\mathrm{C}$-U extension of $\Phi$ iff $S$ is maximal conflict-free set closed under $\mathcal{R}_{s}$;

- $S \subseteq \mathrm{AR}_{S}$ is a CF-S extension of $\Phi$ iff $S$ is a maximal conflict-free set;

- $S \subseteq \mathrm{AR}_{S}$ is an $\mathrm{S}-\mathrm{S}$ extension of $\Phi$ iff $S$ is a maximal safe set; and

- $S \subseteq \mathrm{AR}_{S}$ is a C-S extension of $\Phi$ iff $S$ is a maximal conflict-free set closed under $\mathcal{R}_{s}$.

The primary motivation for analyzing semantics by category is to maintain the consistency of such analysis. If the analysis were carried out without considering the distinction among the strengthened, weakened, and unchanged arguments, then two possibly undesirable situations could arise: $(i)$ an unnecessary duplication of the semantic analysis, since the relationships have already been analyzed and their effects propagated through the labeling process; and (ii) an inadequate semantic analysis that arrives at an unnecessarily restricted set of acceptable arguments; that is, an argument may conflict with another argument weakening it but not defeating it completely. In this latter case, if we analyze the set of arguments as a set without categories, the weakened argument would be discarded; however, after analyzing the argumentation graph, a weakened argument still may have enough strength to cast doubt on a particular decision or at least be part of an explanation.

We thus consider each category of arguments independently of the others, defining extensions for each of them. In this sense, we construct extensions according to the status of the argument, providing us with more information about them. That is, acceptable arguments from the weakened class are those for which important reasons against them exist, while strengthened arguments are those 


\begin{tabular}{|c||c|}
\hline Value-based Extensions & Classical Extensions \\
\hline \hline CF-W & \\
CF-U & d-Preferred \\
CF-S & \\
\hline S-W & \\
S-U & s-Preferred \\
S-S & \\
\hline C-W & \\
C-U & c-Preferred \\
C-S & \\
\hline
\end{tabular}

Table 4: Relationship between Value-based and Classical Extensions

that were mostly supported and that their opponents were not relevant. Finally, the unchallenged set contains both arguments that are not attacked and arguments for which supporters neutralized the attackers. Neutralized arguments are not considered to be part of any extensions since they do not provide any valid knowledge to give a solution to the problem raised.

As we mentioned before, both conflict and support relations are analyzed, taking into account their effect and the obtained defeated arguments. Thus, we analyze the conflict-free and safe notions within each class to preserve the arguments' category. Nevertheless, it is useful to highlight that the extensions that emerge from the strengthened, weakened, and unchallenged classes make up a large set of acceptable arguments. The idea of categorizing accepted groups of statements is useful when making decisions; a decision supported by strengthened arguments is different from a decision based on weakened ones. This classification gives us an intuition of what happened within the discussion and provides us with a degree of confidence about a given decision.

Table 4 presents the relation between an extension in the classical semantics and those introduced in our formalism. Essentially, this relation is based on the properties that they possess: maximality, defense, conflict-freeness, and safety. For example, the d-preferred extension is the maximal conflict-free set that defends all its elements, as are the CF-W, CF-U, and CF-S extensions. Thus, the same analysis can be carried over the other extensions. We do not intend to study here the possible inclusion or equivalence relationship between the extensions mentioned above. Instead, we wish to highlight how a set of extensions in our formalism has the same properties of a classic extension, based on a more refined analysis and a categorization of the arguments based on its attributes.

In the following proposition, the connection between the extensions defined in this work is formalized.

Proposition 4 Let $\Psi=\langle\Theta, A, v\rangle$ be an $L-B A F$, and $\mathrm{AR}_{w}, \mathrm{AR}_{s}$, and $\mathrm{AR}_{u}$ be the sets of weakened, strengthened, and unchallenged arguments associated with $\Psi$. Then:

(i) Any $S$-W (resp. S-S, S-U) extension is also a CF-W (resp. CF-S, CF-U) extension;

(ii) Any CF-W (resp. CF-S, CF-U) extension closed under $\mathcal{R}_{s}$ is also a $S-W$ (resp. S-S, S-U) extension; and

(iii) Any $S$-W (resp. S-S, $S$ - $U$ ) extension closed under $\mathcal{R}_{\text {s }}$ is also a $C$-W (resp. $C$-S, $C$ - $U$ ) extension.

Furthermore, we establish the condition for a labeled argumentation framework to be equivalent to a bipolar argumentation framework. That is, the extension obtained in our framework coincides 


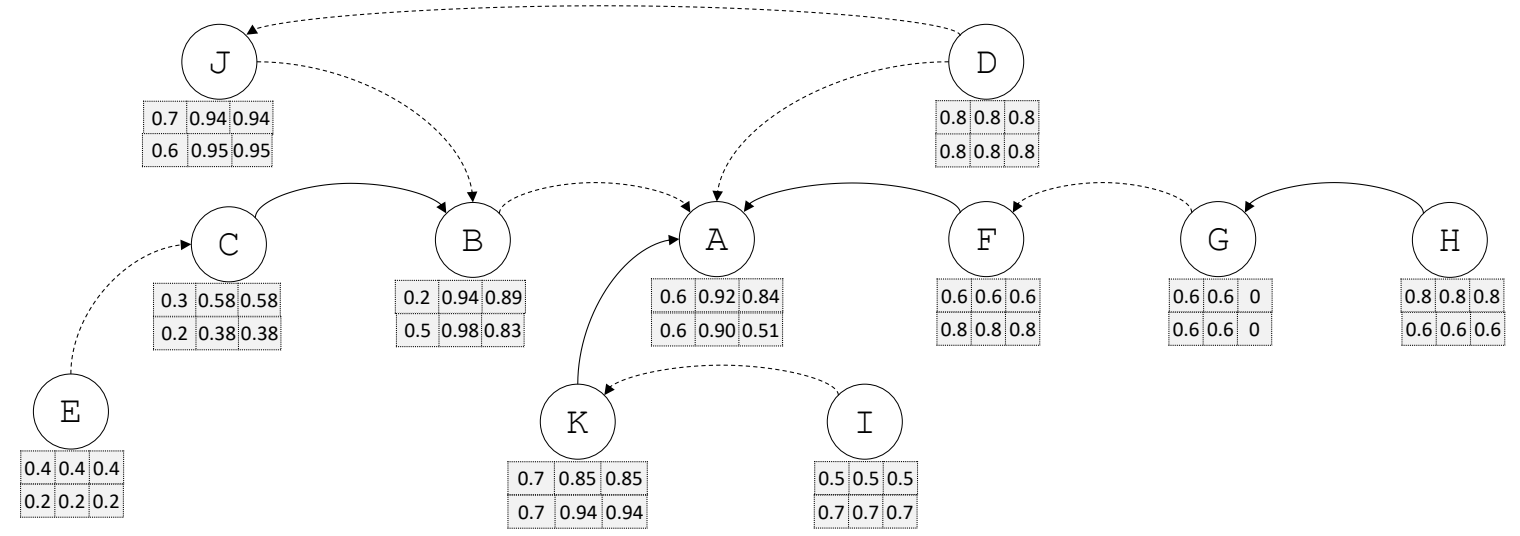

Figure 13: Labeled bipolar argumentation graph.

with the extension obtained for the underlying bipolar argumentation framework. To do this, we first establish the following results.

Proposition 5 Let $\Psi=\langle\Theta, \mathbf{A}, v\rangle$ be an $L-B A F, G_{\Psi}$ be the corresponding labeled bipolar argumentation graph, and $\mathrm{AR}_{w}, \mathrm{AR}_{s}, \mathrm{AR}_{u}$, and $\mathrm{AR}_{n}$ be the sets of weakened, strengthened, unchallenged, and neutralized arguments associated with $\Psi$, respectively. If $\mathrm{AR}_{w}=\emptyset$ and all arguments $\mathrm{A} \in G_{\Psi}$ are such that $\alpha^{\mathrm{A}}=\top$ when $(\overrightarrow{\mathrm{A}})=\emptyset$, and $\alpha^{\mathrm{A}}=\perp$ when $(\mathrm{A}) \neq \emptyset$, then

(i) $\mathrm{AR}_{S}$ and $\mathrm{AR}_{u}$ are conflict-free;

(ii) $\mathrm{AR}_{s} \cup \mathrm{AR}_{u} \cup \mathrm{AR}_{n}=\mathrm{AR}$.

Theorem 3 Let $\Psi=\langle\Theta, \mathbf{A}, v\rangle$ be an $L-B A F$, and $G_{\Psi}$ be the corresponding labeled bipolar argumentation graph. If all arguments $\mathrm{A} \in G$ are such that $\alpha^{\mathrm{A}}=\top$ when $\overrightarrow{(\mathrm{A})}=\emptyset$, and $\alpha^{\mathrm{A}}=\perp$ when $\overrightarrow{\mathrm{A}}) \neq \emptyset$, then

(i) $\mathrm{AR}_{u} \cup \mathrm{AR}_{s}$ is the d-preferred extension;

(ii) If $\mathrm{AR}_{s} \cup \mathrm{AR}_{u}$ is safe, then $\mathrm{AR}_{s} \cup \mathrm{AR}_{u}$ is the s-preferred extension; and

(iii) If $\mathrm{AR}_{s} \cup \mathrm{AR}_{u}$ is closed under $\mathcal{R}_{s}$, then $\mathrm{AR}_{s} \cup \mathrm{AR}_{u}$ is the c-preferred extension.

The following example illustrates these concepts.

Example 5 Continuing with Example 4, we classify the arguments that belong to AR according to Definition 13 as follows:

$$
\begin{aligned}
& \mathrm{AR}_{n}=\{\mathrm{G}\} \text { (neutralized), } \\
& \mathrm{AR}_{w}=\{\mathrm{A}\}(\text { weakened }), \\
& \mathrm{AR}_{s}=\{\mathrm{C}, \mathrm{B}, \mathrm{J}, \mathrm{K}\}(\text { strengthened }), \text { and } \\
& \mathrm{AR}_{u}=\{\mathrm{D}, \mathrm{E}, \mathrm{H}, \mathrm{I}, \mathrm{F}\} \text { (unchallenged) } .
\end{aligned}
$$


We refine the analysis over each set by applying Definition 15 to obtain the acceptability extensions specifying the sets of acceptable arguments. To do this, we first establish a preference function used to identify an order between arguments of AR as follows:

$$
\text { for all } \mathrm{A}, \mathrm{B} \in \mathrm{AR} \mid \mathrm{A} \succ \mathrm{B} \text { iff } \delta_{\mathrm{X}}^{\mathrm{A}}>\delta_{\mathrm{X}}^{\mathrm{B}} \text { for each } \mathrm{X} \in \mathbf{A} \text {. }
$$

The set $\mathrm{AR}_{w}=\{\mathrm{A}\}$ is a CF-W, S-W and C-W extension. The set $\mathrm{AR}_{s}=\{\mathrm{C}, \mathrm{B}, \mathrm{K}, \mathrm{J}\}$ is a CF-S and $S-S$ extension, but it is not $C-S$ extension-the relation $\mathrm{AR}_{s}$ is not closed under $\mathcal{R}_{s}$ because $\mathrm{B}$ supports $\mathrm{A}$, which does not belong to $\mathrm{AR}_{s}$. Observe that $\mathrm{C}$ attacks $\mathrm{B}$ in $\mathrm{AR}_{s}$, but $\mathrm{C} \nsucc \mathrm{B}$ because the accuracy and reliability of $\mathrm{B}$ are better than the accuracy and reliability associated with $\mathrm{C}$ (the accuracy and reliability of $\mathrm{B}$ are 0.89 and 0.83 , while for $\mathrm{C}$ they are 0.58 and 0.38 , respectively). Based on this, also $\mathrm{AR}_{s}$ is safe since J supports $\mathrm{B}$, which is attacked by $\mathrm{C}$; but, as we explained before, the negative effect of the attack relation is lessened by the support of $\mathrm{J}$.

The set $\mathrm{AR}_{u}=\{\mathrm{D}, \mathrm{E}, \mathrm{H}, \mathrm{I}, \mathrm{F}\}$ is a CF-U extension, but it is neither an $S-S$ nor a $C-S$ extension since $\mathrm{AR}_{u}$ is not closed under $\mathcal{R}_{s}$ because $\mathrm{D}$ supports $\mathrm{A}$, which does not belong to $\mathrm{AR}_{u}$. Furthermore, $\mathrm{D}$ supports $\mathrm{A}$, which is attacked by $\mathrm{F} ; \mathrm{F} \succ \mathrm{A}$ because the reliability of $\mathrm{F}$ is better than that of $\mathrm{A}$ (the reliability of $\mathrm{A}$ is 0.51 , and for $\mathrm{F}$ it is 0.8 ).

Let us observe that if we take into account a set of arguments resulting from the union of the strengthened, weakened, and unchallenged sets, and perform the semantic analysis following the intuition presented in Definition 15, we have that the maximal conflict-free set is $S_{1}=$ $\{\mathrm{D}, \mathrm{E}, \mathrm{H}, \mathrm{I}, \mathrm{F}, \mathrm{C}, \mathrm{B}, \mathrm{K}, \mathrm{J}\}$, while no arguments that are safe and closed under support are found. Thus, argument A cannot be considered to be an acceptable argument; however, it has non-neutralized features, yielding a contradiction. This result arises by merging the global semantic analysis performed by the labeling process with the local semantic analysis, taking into account relationships individually through the concept of conflict-free and safe.

Next, we analyze how the interactions between arguments are reflected in the labels representing the features associated with them. We also explore some properties of acceptable arguments.

\section{Labeling Properties}

Recently, a set of properties have been proposed in different works that allow to better understand the behavior of the different value-based semantics. The following postulates are satisfied by all the valuations defined according to the labeling process. In general, these postulates describe the behavior of valuations associated with arguments in our framework; they are studied by Amgoud et al. (2013), Amgoud and Ben-Naim (2018), Potyka (2018), and Matt \& Toni (2008).

First, our labeling procedure defined over a set of arguments should analyze the identity of the arguments (represented through the original valuation) and consider the basis of the support and attack between arguments (represented through the strengthened and overall valuations, respectively). So, the first postulate says that two equivalent labeled bipolar argumentation frameworks should give rise to equivalent families of valid labelings (models). Towards this end, first we introduce the notion of isomorphism between two $L-B A F s$, which is used to connect them.

Definition 16 (Isomorphism) Let $\Psi=\langle\Theta, \mathbf{A}, v\rangle$ and $\Psi^{\prime}=\left\langle\Theta^{\prime}, \mathbf{A}^{\prime}, v^{\prime}\right\rangle$ be two L-BAFs where $\Theta=$ $\left\langle\mathrm{AR}, \mathcal{R}_{a}, \mathcal{R}_{s}\right\rangle$ and $\Theta^{\prime}=\left\langle\mathrm{AR}^{\prime}, \mathcal{R}_{a}^{\prime}, \mathcal{R}_{s}^{\prime}\right\rangle$ are the underlying BAFs, $v$ and $v^{\prime}$ the valuation functions, and $\rho$ and $\rho^{\prime}$ the posture functions. An isomorphism from $\Psi$ to $\Psi^{\prime}$ is a bijective function $h: \mathrm{AR} \longrightarrow \mathrm{AR}^{\prime}$ such that: 
(i) $\forall \mathrm{A} \in \mathrm{AR}, v(\mathrm{~A})=v^{\prime}(h(\mathrm{~A}))$,

(ii) $\forall \mathrm{A} \in \mathrm{AR}, \rho(\mathrm{A})=\rho^{\prime}(h(\mathrm{~A}))$,

(iii) $\forall \mathrm{A}, \mathrm{B} \in \mathrm{AR},(\mathrm{A}, \mathrm{B}) \in \mathcal{R}_{a}$ iff $(h(\mathrm{~A}), h(\mathrm{~B})) \in \mathcal{R}_{a}^{\prime}$, and

(iv) $\forall \mathrm{A}, \mathrm{B} \in \mathrm{AR},(\mathrm{A}, \mathrm{B}) \in \mathcal{R}_{s}$ iff $(h(\mathrm{~A}), h(\mathrm{~B})) \in \mathcal{R}_{s}^{\prime}$.

In what follows, we will introduce a set of postulates and analyze them concerning the semantics presented in this formalism. To clarify their statements, when no confusion arises, we will simplify the description by omitting to mention the context represented by the $L-B A F \Psi=\langle\Theta, \mathbf{A}, v\rangle$ and its underlying $B A F \Theta=\left\langle\mathrm{AR}, \mathcal{R}_{a}, \mathcal{R}_{s}\right\rangle$.

Postulate 1 (Abstraction) Given two L-BAFs $\Psi=\langle\Theta, \mathbf{A}, v\rangle$ and $\Psi^{\prime}=\left\langle\Theta^{\prime}, \mathbf{A}^{\prime}, v^{\prime}\right\rangle$, and an isomorphism $h: \Psi \longrightarrow \Psi^{\prime}$, then the following property holds:

For all $\mathrm{A} \in \mathrm{AR},\left\langle\alpha^{\mathrm{A}}, \mu^{\mathrm{A}}, \delta^{\mathrm{A}}\right\rangle \in \Lambda_{\Psi}$ if and only if $\left\langle\alpha^{h(\mathrm{~A})}, \mu^{h(\mathrm{~A})}, \delta^{h(\mathrm{~A})}\right\rangle \in \Lambda_{\Psi^{\prime}}$

In the following, we assume an $L-B A F \quad \Psi=\langle\Theta, \mathbf{A}, v\rangle$, the underlying $B A F$ $\Theta=\left\langle\right.$ AR, $\left.\mathcal{R}_{a}, \mathcal{R}_{s}\right\rangle$, the corresponding argumentation graph for $\Theta$ denoted as $\mathrm{G}_{\Theta}$, and a valid labeling for $\mathrm{G}_{\Theta}$ denoted with $\lambda(\Theta)$.

The second postulate refers to the notion of independence between valuations, which simply means that one can be computed without the other. The postulate then states that the answer to the question of whether an argument $A$ is at least as acceptable as an argument $B$ should be independent of any argument $C$ that is neither connected to $A$ nor to $B$; that is, there is no path from $C$ to $A$ nor $B$ (without considering the direction of the edges).

Postulate 2 (Independence) Let $\mathrm{A}$ and $\mathrm{B}$ be two arguments in $\mathrm{AR}$ that are not connected in $\mathrm{G}_{\Theta}$. Then, the valuations $\left\langle\alpha^{\mathrm{A}}, \mu^{\mathrm{A}}, \delta^{\mathrm{A}}\right\rangle,\left\langle\alpha^{\mathrm{B}}, \mu^{\mathrm{B}}, \delta^{\mathrm{B}}\right\rangle \in \lambda(\Theta)$ associated with $\mathrm{A}$ and $\mathrm{B}$, respectively, are independent.

The third postulate says that if A is attacked with at most the same strength as B, and supported with at least the same strength as B, then A should be at least as strong as B.

Postulate 3 (Monotony) Let $\left\langle\alpha^{\mathrm{A}}, \mu^{\mathrm{A}}, \delta^{\mathrm{A}}\right\rangle,\left\langle\alpha^{\mathrm{B}}, \mu^{\mathrm{B}}, \delta^{\mathrm{B}}\right\rangle \in \lambda(\Theta)$ be the valuations associated with two arguments $\mathrm{A}$ and $\mathrm{B}$ respectively. It holds that if

(i) $\alpha^{\mathrm{A}}=\alpha^{\mathrm{B}}$,

(ii) $\odot_{j=1}^{m} \delta^{\mathrm{A}_{j}} \geq \odot_{j=1}^{r} \delta^{\mathrm{B}_{j}}$ with $\mathrm{A}_{j} \in \overrightarrow{(\mathrm{A})}, 1 \leq j \leq m$, and $\mathrm{B}_{j} \in \overrightarrow{(\mathrm{B})}, 1 \leq j \leq r$,

(iii) $\otimes_{k=1}^{n} \delta^{\mathrm{x}_{k}} \leq \otimes_{k=1}^{s} \delta^{\mathrm{Y}_{k}}$ with $\mathrm{X}_{k} \in \overrightarrow{(\mathrm{A})}, 1 \leq k \leq n$, and $\mathrm{Y}_{k} \in \overrightarrow{(\mathrm{B})}, 1 \leq k \leq s$,

then $\delta^{\mathrm{A}} \geq \delta^{\mathrm{B}}$.

The directionality postulate states that the overall valuation of an argument should depend only on its incoming edges, and not on the arguments it attacks or supports; i.e., the attacks and supports are directed relations, and the attacker (supporter) influences the attacked (supported). 
Postulate 4 (Directionality) Let $\left\langle\alpha^{\mathrm{A}}, \mu^{\mathrm{A}}, \delta^{\mathrm{A}}\right\rangle \in \lambda(\Theta)$ be a valuation associated with argument $\mathrm{A}$. Then:

(i) If there is no path in $\mathrm{G}_{\Theta}$ arriving to $\mathrm{A}$, then $\alpha^{\mathrm{A}}=\mu^{\mathrm{A}}=\delta^{\mathrm{A}}$.

(ii) If $\overrightarrow{(\mathrm{A})} \neq \emptyset$ and $\overrightarrow{(\mathrm{A})}=\emptyset$, then $\alpha^{\mathrm{A}} \leq \mu^{\mathrm{A}}=\delta^{\mathrm{A}}$.

(iii) If $\overrightarrow{(\mathrm{A})}=\emptyset$ and $\overrightarrow{(\mathrm{A})} \neq \emptyset$, then $\alpha^{\mathrm{A}}=\mu^{\mathrm{A}} \geq \delta^{\mathrm{A}}$.

(iv) If $\overrightarrow{(\mathrm{A})} \neq \emptyset$ and $\overrightarrow{(\mathrm{A})} \neq \emptyset$, then $\alpha^{\mathrm{A}} \leq \mu^{\mathrm{A}} \geq \delta^{\mathrm{A}}$.

The next postulate, called equivalence, ensures that the overall strength of an argument depends only on the original valuations of the arguments and the overall valuations of its (direct) attackers and supporters.

Postulate 5 (Equivalence) Let $\left\langle\alpha^{\mathrm{A}}, \mu^{\mathrm{A}}, \delta^{\mathrm{A}}\right\rangle,\left\langle\alpha^{\mathrm{B}}, \mu^{\mathrm{B}}, \delta^{\mathrm{B}}\right\rangle \in \lambda(\Theta)$ be the valuations associated with two arguments $\mathrm{A}$ and $\mathrm{B}$, respectively. Then, if it holds that

(i) $\alpha^{\mathrm{A}}=\alpha^{\mathrm{B}}$,

(ii) there exists a bijective function $f: \overrightarrow{\mathrm{A}}) \longrightarrow \overrightarrow{\mathrm{B}})$ such that for all $\mathrm{X} \in(\overrightarrow{\mathrm{A}}) \mu^{\mathrm{X}}=\mu^{f(\mathrm{x})}$,

(iii) there exists a bijective function $g: \overrightarrow{(\mathrm{A})} \longrightarrow \overrightarrow{(\mathrm{B})}$ such that for all $\mathrm{Y} \in \overrightarrow{(\mathrm{A})} \delta^{\mathrm{Y}}=\delta^{g(\mathrm{Y})}$,

then $\left\langle\alpha^{\mathrm{A}}, \mu^{\mathrm{A}}, \delta^{\mathrm{A}}\right\rangle=\left\langle\alpha^{\mathrm{B}}, \mu^{\mathrm{B}}, \delta^{\mathrm{B}}\right\rangle$, which means that $\mathrm{A}$ and $\mathrm{B}$ are equivalent.

The following local property is concerned with the direct supporters and attackers (defenders) of arguments. The strengthened label associated with an argument represents how strong its supports are. Thus, when no attacking arguments exist to provide a weakening for an argument, its valuation is strengthened by the supporting arguments. On the other hand, the quality of a direct attacker depends on the quality of its attackers, and so on. Thus, the overall valuation associated with an argument represents how strong its defenders and attackers are.

Postulate 6 (Quality Precedence) Given the valuations $\left\langle\alpha^{\mathrm{A}}, \mu^{\mathrm{A}}, \delta^{\mathrm{A}}\right\rangle,\left\langle\alpha^{\mathrm{B}}, \mu^{\mathrm{B}}, \delta^{\mathrm{B}}\right\rangle \in \lambda(\Theta)$ associated with two arguments $\mathrm{A}$ and $\mathrm{B}$, respectively. When $\alpha^{\mathrm{A}} \geq \alpha^{\mathrm{B}}$, we have that:

(i) If the strengths associated with the supporters of $\mathrm{A}$ and $\mathrm{B}$ verify that

$\odot_{j=1}^{m} \delta^{\mathrm{A}_{j}}>\odot_{j=1}^{r} \delta^{\mathrm{B}_{j}}$ with $\mathrm{A}_{j} \in \overrightarrow{(\mathrm{A})}$ and $\mathrm{B}_{j} \in(\overrightarrow{\mathrm{B}})$, then

$\delta^{\mathrm{A}}>\delta^{\mathrm{B}}$ when $\overrightarrow{(\mathrm{A})}=\emptyset$ and $\overrightarrow{(\mathrm{B})}=\emptyset$.

(ii) If the strengths associated with the attackers of $\mathrm{A}$ and $\mathrm{B}$ verify that

$\otimes_{k=1}^{n} \delta^{\mathrm{X}_{k}}<\otimes_{k=1}^{s} \delta^{\mathrm{Y}_{k}}$ with $\mathrm{X}_{k} \in \overrightarrow{(\mathrm{A})}$ and $\mathrm{Y}_{k} \in \overrightarrow{(\mathrm{B})}$, then

$\delta^{\mathrm{A}}>\delta^{\mathrm{B}}$ when $(\overrightarrow{\mathrm{A}})=\emptyset$ and $(\mathrm{B})=\emptyset$.

The previous postulates suggest the introduction of three other postulates: neutrality, reinforcement, and stability. In particular, neutrality states that, if the strengths associated with the supporting and attacking arguments are equal to $\perp$, then these relations cannot produce an effect. 
Postulate 7 (Neutrality) For any argument $\mathrm{A} \in \mathrm{AR}$ where $\left\langle\alpha^{\mathrm{A}}, \mu^{\mathrm{A}}, \delta^{\mathrm{A}}\right\rangle \in \lambda(\Theta)$ we have that: if $\odot_{j=1}^{m} \delta^{\mathrm{A}_{j}}=\otimes_{k=1}^{n} \delta^{\mathrm{x}_{k}}=\perp$ with $\mathrm{A}_{j} \in \overrightarrow{(\mathrm{A})}, 1 \leq j \leq m$, and $\mathrm{X}_{k} \in \overrightarrow{(\mathrm{A})}, 1 \leq k \leq n$, then $\delta^{\mathrm{A}}=\alpha^{\mathrm{A}}$.

The reinforcement postulate states that any argument becomes stronger if the quality of its attackers is reduced or the quality of its supporters is increased.

Postulate 8 (Reinforcement) For any argument $\mathrm{A} \in \mathrm{AR}$ and $\left\langle\alpha^{\mathrm{A}}, \mu^{\mathrm{A}}, \delta^{\mathrm{A}}\right\rangle \in \lambda(\Theta)$ then $\delta^{\mathrm{A}}>\alpha^{\mathrm{A}}$ iff $\odot_{j=1}^{m} \delta^{\mathrm{A}_{j}}>\otimes_{k=1}^{n} \delta^{\mathrm{x}_{k}}$, where $\mathrm{A}_{j} \in \overrightarrow{(\mathrm{A})}, 1 \leq j \leq m$ and $\mathrm{X}_{k} \in \overrightarrow{(\mathrm{A})}, 1 \leq k \leq n$.

The stability postulate states that if an argument is neither attacked nor supported, its overall valuation should be equal to its original valuation.

Postulate 9 (Stability) For any argument A where $\left\langle\alpha^{\mathrm{A}}, \mu^{\mathrm{A}}, \delta^{\mathrm{A}}\right\rangle \in \lambda(\Theta)$ if $\overrightarrow{(\mathrm{A})}=\emptyset$ and $(\overrightarrow{\mathrm{A}})=\emptyset$ we have that $\delta^{\mathrm{A}}=\alpha^{\mathrm{A}}$.

The following two postulates are centered on weakening and strengthening between arguments. Weakening states that if the strength of the attacking arguments overcomes the strength of the supporting arguments, then the affected argument loses its original attributes. Intuitively, supporters are not sufficient for counter-balancing attackers; however, they may mitigate the global loss due to attacks.

Postulate 10 (Weakening) For any argument A where $\left\langle\alpha^{\mathrm{A}}, \mu^{\mathrm{A}}, \delta^{\mathrm{A}}\right\rangle \in \lambda(\Theta)$, we have:

(i) If $\otimes_{k=1}^{n} \delta^{\mathrm{x}_{k}}>\odot_{j=1}^{m} \delta^{\mathrm{A}_{j}}$ with $\mathrm{X}_{k} \in \overrightarrow{(\mathrm{A})}, 1 \leq k \leq n$, and $\mathrm{A}_{j} \in\left(\overrightarrow{\mathrm{A})}, 1 \leq j \leq m\right.$, then $\alpha^{\mathrm{A}}>\delta^{\mathrm{A}}$.

(ii) If $\overrightarrow{(\mathrm{A})} \neq \emptyset$ and $\overrightarrow{(\mathrm{A})}=\emptyset$, then $\alpha^{\mathrm{A}} \geq \delta^{\mathrm{A}}$.

On the other hand, strengthening states that if the strength of the supporting arguments overcomes the strength of the attacking arguments, then the affected argument increases the original features. Indeed, attacks are not sufficient for counter-balancing supports; however, they may mitigate the global gain due to supports.

Postulate 11 (Strengthening) For any argument A where $\left\langle\alpha^{\mathrm{A}}, \mu^{\mathrm{A}}, \delta^{\mathrm{A}}\right\rangle \in \lambda(\Theta)$, we have that:

(i) If $\otimes_{k=1}^{n} \delta^{\mathrm{x}_{k}} \leq \odot_{j=1}^{m} \delta^{\mathrm{A}_{j}}$ with $\mathrm{X}_{k} \in \overrightarrow{(\mathrm{A})}, 1 \leq k \leq n$, and $\mathrm{A}_{j} \in \overrightarrow{(\mathrm{A})}, 1 \leq j \leq m$, then $\alpha^{\mathrm{A}} \leq \delta^{\mathrm{A}}$.

(ii) If $\overrightarrow{(\mathrm{A})}=\emptyset$, then $\alpha^{\mathrm{A}} \leq \delta^{\mathrm{A}}$.

Strengthening soundness and weakening soundness express that any difference between the original valuation and the overall valuation of an argument is caused by some supporting or attacking argument. This property is called causality.

Postulate 12 (Causality) For any argument A where $\left\langle\alpha^{\mathrm{A}}, \mu^{\mathrm{A}}, \delta^{\mathrm{A}}\right\rangle \in \lambda(\Theta)$. Then:

(i) If $\mu^{\mathrm{A}} \neq \delta^{\mathrm{A}}$ there exists an argument $\mathrm{B} \in \mathrm{AR}$ such that $\delta^{\mathrm{B}} \neq \perp$ with $\mathrm{B} \in \overrightarrow{(\mathrm{A})}$. 
(ii) If $\alpha^{\mathrm{A}} \neq \mu^{\mathrm{A}}$ there exists an argument $\mathrm{B} \in \mathrm{AR}$ such that $\delta^{\mathrm{B}} \neq \perp$ with $\mathrm{B} \in(\overrightarrow{\mathrm{A}})$.

Neutralization expresses that if an argument $\mathrm{A}$ is attacked by $\mathrm{X}$ and supported by $\mathrm{Y}$, and the overall valuations of $\mathrm{X}$ and $\mathrm{Y}$ are identical, then they neutralize each other with respect to $\mathrm{A}$.

Postulate 13 (Neutralization) For any argument $\mathrm{A} \in \mathrm{AR}$ where $\left\langle\alpha^{\mathrm{A}}, \mu^{\mathrm{A}}, \delta^{\mathrm{A}}\right\rangle \in \lambda(\Theta)$, if it holds that $\odot_{j=1}^{m} \delta^{\mathrm{A}_{j}}=\otimes_{k=1}^{n} \delta^{\mathrm{x}_{k}}$ with $\mathrm{A}_{j} \in \overrightarrow{(\mathrm{A})}, 1 \leq j \leq m$, and $\mathrm{x}_{k} \in \overrightarrow{(\mathrm{A})}, 1 \leq k \leq n$, then $\alpha^{\mathrm{A}}=\delta^{\mathrm{A}}$.

The following postulate, called proportionality, states that the stronger the target of an attack, the weaker its intensity.

Postulate 14 (Proportionality) For any arguments A, B where $\left\langle\alpha^{\mathrm{A}}, \mu^{\mathrm{A}}, \delta^{\mathrm{A}}\right\rangle,\left\langle\alpha^{\mathrm{B}}, \mu^{\mathrm{B}}, \delta^{\mathrm{B}}\right\rangle \in \lambda(\Theta)$, if

(i) $\alpha^{\mathrm{A}}>\alpha^{\mathrm{B}}$,

(ii) $\stackrel{\overrightarrow{\mathrm{B}})}{\overrightarrow{\mathrm{B}}}=\emptyset$ or $\odot_{j=1}^{m} \delta^{\mathrm{A}_{\mathrm{j}}} \geq \odot_{k=1}^{r} \delta^{\mathrm{B}_{\mathrm{k}}}$ with $\mathrm{A}_{j} \in(\overrightarrow{\mathrm{A}}), 1 \leq j \leq m$, and $\mathrm{B}_{k} \in \overrightarrow{(\mathrm{B})}, 1 \leq k \leq n$,

(iii) $\otimes_{j=1}^{m} \delta^{\mathrm{x}_{j}}=\otimes_{k=1}^{n} \delta^{\mathrm{Y}_{\mathrm{k}}}$ with $\mathrm{X}_{j} \in \overrightarrow{(\mathrm{A})}, 1 \leq j \leq m$, and $\mathrm{Y}_{k} \in \overrightarrow{(\mathrm{B})}, 1 \leq k \leq n$, and

(iv) $\delta^{\mathrm{A}}>\perp$.

then $\delta^{\mathrm{A}}>\delta^{\mathrm{B}}$.

Finally, all postulates are compatible; i.e., it is possible to satisfy all of them with a single labeling process.

Theorem 4 Any L-BAF instantiation satisfies the following postulates: Abstraction, Independence, Monotony, Directionality, Equivalence, Quality Precedence, Neutrality, Reinforcement, Stability, Weakening, Strengthening, Causality, Neutralization, and Proportionality.

\section{An Extended Analysis of the Running Example}

In this section, we return to the example from Section 1 to present a full account and complete representation using all elements of the proposed formalism. Recall that in this scenario, parents are analyzing arguments in favor and against the selection of schools according to their preferences. Therefore, the arguments endorsing specific characteristics have different preference valuations representing the parents' preference regarding the arguments' topics, as well as the social valuation that represents society's support (in the form of engagement via a social platform) of the information advanced by the arguments. Thus, this scenario can be represented and analyzed by our formalism as follows:

Let $\Psi=\langle\Theta, \mathbf{A}, v\rangle$ be an $L-B A F$ with underlying $B A F \Theta=\left\langle\mathrm{AR}, \mathcal{R}_{a}, \mathcal{R}_{s}\right\rangle$, where:

- $A R=\{A ; B ; C ; D ; E ; F ; G ; H ; I ; J ; K\}$,

- $\mathcal{R}_{a}=\{(\mathrm{B}, \mathrm{A}),(\mathrm{D}, \mathrm{A}),(\mathrm{I}, \mathrm{B}),(\mathrm{K}, \mathrm{C}),(\mathrm{H}, \mathrm{F})\}$,

- $\mathcal{R}_{s}=\{(\mathrm{C}, \mathrm{B}),(\mathrm{E}, \mathrm{D}),(\mathrm{F}, \mathrm{A}),(\mathrm{G}, \mathrm{F}),(\mathrm{I}, \mathrm{F}),(\mathrm{J}, \mathrm{A})\}$, 
- $\mathbf{A}=\{\mathrm{A}, \mathrm{B}\}$ is the set of two algebras, where $\mathrm{A}$ represents preference and $\mathrm{B}$ represents social rating for each argument in $\mathrm{AR}$. Both algebras are defined below.

- $v$ is a function that assigns to each argument in AR a tuple in $A \times B$ representing the preference and social rating associated with that argument.

The algebras are defined as follows:

Algebra A: The domain of labels $\mathfrak{L}_{1}$ of algebra $\mathrm{A}$ is the real interval $[0,1]$ representing a normalized preference valuation with $T=1$ and $\perp=0$. The preference over the arguments represents the agent's preference regarding specific issues that influence the decisions that it considers. The operators of accumulation, strengthening, and weakening defined to manipulate this feature are introduced as follows:

\begin{tabular}{|c|c|}
\hline \multicolumn{2}{|c|}{ Preference } \\
\hline $\begin{array}{l}\gamma \quad \odot \quad \begin{array}{ll}\gamma & \multicolumn{1}{c}{\odot} \\
\{ & \text { if } \gamma=\beta=0 \\
\frac{\gamma \beta}{\gamma+\beta-\gamma \beta} & \text { otherwise. }\end{array}\end{array}$ & $\begin{array}{l}\text { Determines the accumulation valuation } \\
\text { as the gradual combination of the valu- } \\
\text { ation associated with the involved argu- } \\
\text { ments applying a gradual conjunction. }\end{array}$ \\
\hline$\gamma \otimes \beta=\min (\gamma, \beta)$ & $\begin{array}{l}\text { Yields the accumulation valuation as } \\
\text { the minimal valuation associated with } \\
\text { the involved arguments applying the } \\
\text { worst-link principle; thus, the valuation } \\
\text { that weakens an argument is as good as } \\
\text { the worst argument that attacks it. }\end{array}$ \\
\hline$\gamma \oplus \beta=\min (\gamma+\beta, 1)$ & $\begin{array}{l}\text { The strengthening operation produces a } \\
\text { preference valuation associated with an } \\
\text { argument combining its own preference } \\
\text { and the supporting accumulation mea- } \\
\text { sure associated with it, with an upper } \\
\text { bound. }\end{array}$ \\
\hline$\gamma \ominus \beta=\max (\gamma-\beta, 0)$ & $\begin{array}{l}\text { The preference of its attackers reduces } \\
\text { an argument's preference in a direct de- } \\
\text { creasing way by the weakening opera- } \\
\text { tor, with a lower bound. Thus, the ef- } \\
\text { fects produced by this relationship de- } \\
\text { pend on the quality of the attacking ar- } \\
\text { guments. }\end{array}$ \\
\hline
\end{tabular}

Algebra $\mathrm{B}$ : The domain of labels $\mathfrak{L}_{2}$ of algebra $\mathrm{B}$ is the real interval $[0,1]$ representing a normalized social rating with $T=1$ and $\perp=0$. The social rating attached to the arguments is related to society's support. The operators of support and conflict established to manipulate this feature are defined as follows:

\begin{tabular}{|l|l|}
\hline \multicolumn{2}{|c|}{ Social Valuation } \\
\hline$\gamma \odot \beta=\gamma \beta \quad \begin{array}{l}\text { The determination of the support accu- } \\
\text { mulation valuation is based on the con- } \\
\text { junction of the social valuations corre- } \\
\text { sponding to the arguments that support } \\
\text { it. }\end{array}$ \\
\hline
\end{tabular}




\begin{tabular}{|c|c|c|}
\hline \multicolumn{3}{|c|}{ Social Valuation } \\
\hline$\gamma \otimes \beta=\min (\gamma, \beta)$ & & $\begin{array}{l}\text { The accumulation valuation is the min- } \\
\text { imal among the social rating valua- } \\
\text { tions associated with the attacking ar- } \\
\text { guments; thus, the attack valuation that } \\
\text { weakens an argument is as good as the } \\
\text { worst argument that attacks it. }\end{array}$ \\
\hline$\gamma \oplus \beta=\frac{\gamma+\beta}{1+\gamma \beta}$ & & $\begin{array}{l}\text { This operator reflects the gradual } \\
\text { strengthening of the original social val- } \\
\text { uation associated with an argument } \\
\text { when supporting arguments are consid- } \\
\text { ered. }\end{array}$ \\
\hline$\gamma \ominus \beta=\left\{\begin{array}{l}\frac{\gamma-\beta}{1-\beta} \\
0\end{array}\right.$ & $\begin{array}{l}\text { if } \gamma \geq \beta, \beta \neq 1 \\
\text { otherwise. }\end{array}$ & $\begin{array}{l}\text { The conflict operation identifies the } \\
\text { gradual overall valuation associated } \\
\text { with conflicting arguments where the } \\
\text { effects produced by this relationship de- } \\
\text { pend on the quality of the arguments in- } \\
\text { volved. }\end{array}$ \\
\hline
\end{tabular}

The valuation $v: A R \longrightarrow A \times B$ specifies the preference degree associated with each argument based on the parents' preference, and determines the original (normalized) social valuation associated with each argument (tweet) based on the number of followers, the number of retweets, and the number of likes, as follows:

\begin{tabular}{l|lllllllllll} 
& \multicolumn{10}{|c}{ Argument } \\
$v$ & A & B & C & D & E & F & G & H & I & J & K \\
\hline$\alpha_{1}$ & 0.9 & {$[0.5,0.8]$} & 0.9 & 0.8 & 0.4 & 0.7 & 0.8 & {$[0.4,0.7]$} & 0.6 & 0.8 & 0.6 \\
$\alpha_{2}$ & 0.9 & 0.7 & 0.9 & 0.3 & {$[0.3,0.6]$} & 0.8 & 1 & {$[0.2,0.8]$} & 0.7 & 0.5 & {$[0.4,0.7]$}
\end{tabular}

Next, we analyze the different results obtained when using the classical bipolar approach in comparison with the new formalism.

\section{Comparison with the classical bipolar approach}

Analyzing $L-B A F \Psi$ (cf. Figure 14) using the classical bipolar notions, the set $S=\{\mathrm{E}, \mathrm{H}, \mathrm{I}, \mathrm{J}, \mathrm{K}, \mathrm{D}, \mathrm{G}\}$ is a d-preferred extension. But, $S$ is not safe since G supports $\mathrm{F}$ that is attacked by $\mathrm{H}$ with $\mathrm{H}$ and $\mathrm{G}$ belonging to $S$, or closed under $\mathcal{R}_{s}$ because J supports A, which does not belong to $S$ (which is an admissible set).

Turning to our approach, suppose that the parents are interested in optimizing the argumentation discussion representing their posture over the uncertainty intrinsically associated with the information provided by the domain; this uncertainty is expressed as the uncertainty in parents' perception and the parents' preference tolerance. In this sense, we can establish their posture over the arguments using function $\rho$ as follows:

$$
\begin{array}{ll}
\text { Optimistic Posture } & \text { Pessimistic Posture } \\
\rho_{\mathrm{K}}: \max ([\gamma, \beta]) & \rho_{\mathrm{H}}: \min ([\gamma, \beta]) \\
& \\
\text { Optimistic Leaning Posture } & \text { Pessimistic Leaning Posture } \\
\rho_{\mathrm{E}}: \operatorname{med}(\operatorname{med}([\gamma, \beta]), \beta) & \rho_{\mathrm{B}}: \operatorname{med}(\gamma, \operatorname{med}([\gamma, \beta]))
\end{array}
$$




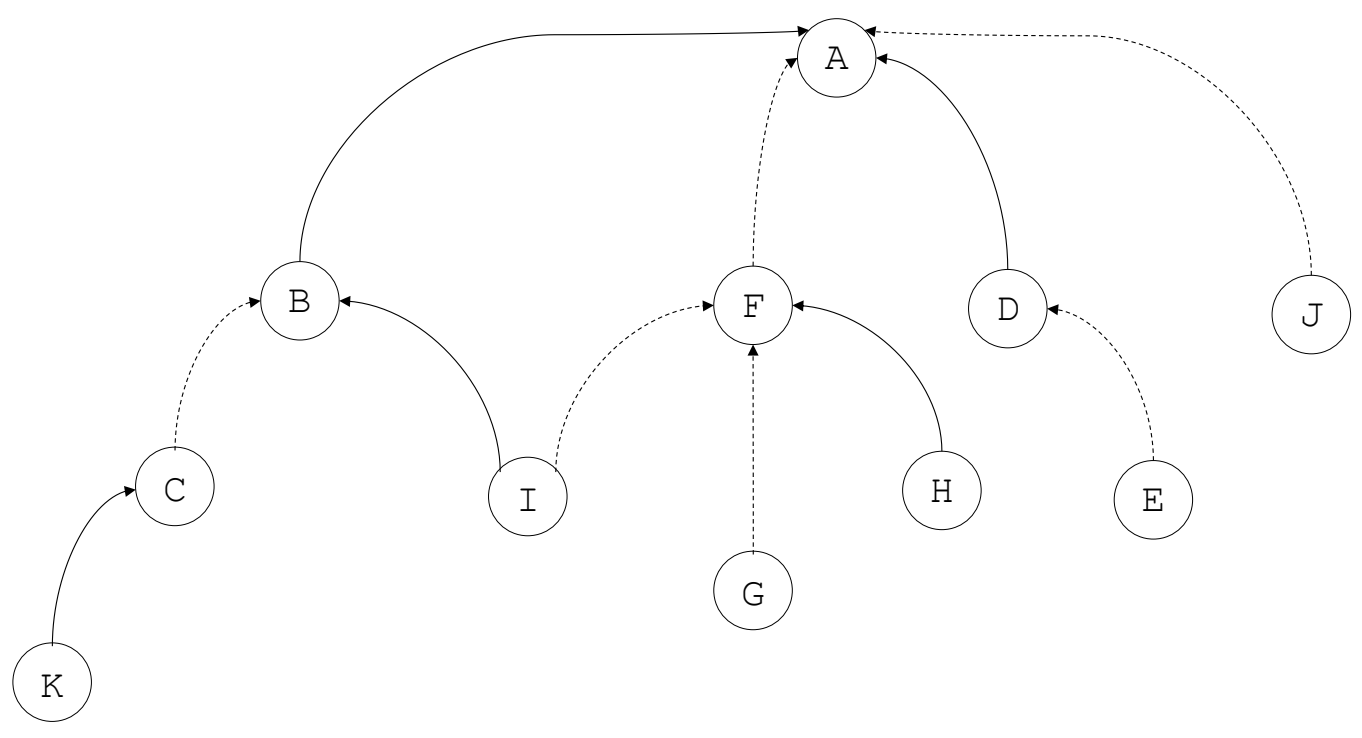

Figure 14: Labeled bipolar argumentation graph.

In this case, we take an optimistic posture over argument $\mathrm{K}$ postulating that the school will close an agreement with the leading universities to increase its students' options and thus secure a better future, while a pessimistic posture is established for argument $\mathrm{H}$ arguing that the classes are large with an extensive extracurricular activity program. Thus, the parents consider the student's future concerning university opportunities to be important, and that the extensive extracurricular activity program helps the student to develop responsibility and skills. On the other hand, they consider an optimistic leaning posture over argument E postulating that the teachers do not have the skills to address this new generation of students, while a pessimistic leaning posture over argument $B$ is fixed arguing that students leave school before completing the last year. Thus, the parents think that the conservative way of teaching is right even though teachers do not use modern resources. Furthermore, they analyze the situation regarding dropouts, realizing that the reasons causing this are foreign to the school.

For reasons of readability, we do not include the position functions that determine the valuations of the arguments with single values. Thus, the final attribute table associated with the arguments involved in the discussion is the following:

\begin{tabular}{l|lllllllllll} 
& \multicolumn{1}{|c}{ Argument } \\
$v$ & A & B & C & D & E & F & G & H & I & J & K \\
\hline$\alpha_{1}$ & 0.9 & 0.58 & 0.9 & 0.8 & 0.4 & 0.7 & 0.8 & 0.4 & 0.6 & 0.8 & 0.6 \\
$\alpha_{2}$ & 0.9 & 0.7 & 0.9 & 0.3 & 0.53 & 0.8 & 1 & 0.2 & 0.7 & 0.5 & 0.7
\end{tabular}

Now, we propagate the valuations associated with each argument following the procedure from Definition 11. Through this process, the system of equations $E Q S$ for the argumentation graph $\mathrm{G}_{\Theta}$ is obtained (cf. Figure 15), and the final labeling argumentation graph $\mathrm{G}_{\Psi}$ (cf. Figure 16) is computed. Clarifying the presentation, we do not include the equations that determine the valuations of the leaf nodes because of their simplicity. 


$$
\left\{\begin{array}{l}
\mathrm{e}_{1}^{\mathrm{X}}: \mu_{\mathrm{X}}^{\mathrm{C}}=\alpha_{\mathrm{X}}^{\mathrm{C}} \\
\mathrm{e}_{2}^{\mathrm{X}}: \delta^{\mathrm{C}}=\alpha_{\mathrm{X}}^{\mathrm{C}} \ominus \delta_{\mathrm{X}}^{\mathrm{K}} \\
\mathrm{e}_{3}^{\mathrm{X}}: \mu^{\mathrm{B}}=\alpha_{\mathrm{X}}^{\mathrm{B}} \oplus \delta_{\mathrm{X}}^{\mathrm{C}} \\
\mathrm{e}_{4}^{\mathrm{X}}: \delta^{\mathrm{B}}=\mu_{\mathrm{X}}^{\mathrm{B}} \ominus\left(\delta_{\mathrm{X}}^{\mathrm{I}} \odot \delta_{\mathrm{X}}^{\mathrm{C}}\right) \\
\mathrm{e}_{5}^{\mathrm{X}}: \mu_{\mathrm{X}}^{\mathrm{F}}=\alpha_{\mathrm{X}}^{\mathrm{F}} \oplus\left(\delta_{\mathrm{X}}^{\mathrm{I}} \odot \delta_{\mathrm{X}}^{\mathrm{G}}\right) \\
\mathrm{e}_{\mathrm{X}}^{\mathrm{X}}: \delta_{\mathrm{X}}^{\mathrm{F}}=\alpha_{\mathrm{X}}^{\mathrm{F}} \oplus\left(\left(\delta_{\mathrm{X}}^{\mathrm{I}} \odot \delta_{\mathrm{X}}^{\mathrm{G}}\right) \ominus \delta_{\mathrm{X}}^{\mathrm{H}}\right) \\
\mathrm{e}_{7}^{\mathrm{X}}: \mu_{\mathrm{X}}^{\mathrm{D}}=\alpha_{\mathrm{X}}^{\mathrm{D}} \oplus \delta_{\mathrm{X}}^{\mathrm{E}} \\
\mathrm{e}_{8}^{\mathrm{X}}: \delta_{\mathrm{X}}^{\mathrm{D}}=\mu_{\mathrm{X}}^{\mathrm{D}} \\
\mathrm{e}_{9}^{\mathrm{X}}: \mu_{\mathrm{X}}^{\mathrm{A}}=\alpha_{\mathrm{X}}^{\mathrm{A}} \oplus\left(\delta_{\mathrm{X}}^{\mathrm{F}} \odot \delta_{\mathrm{X}}^{\mathrm{J}}\right) \\
\mathrm{e}_{10}^{\mathrm{X}}: \delta_{\mathrm{X}}^{\mathrm{A}}=\alpha_{\mathrm{X}}^{\mathrm{A}} \ominus\left(\left(\delta_{\mathrm{X}}^{\mathrm{B}} \otimes \delta_{\mathrm{X}}^{\mathrm{D}}\right) \ominus\left(\delta_{\mathrm{X}}^{\mathrm{F}} \odot \delta_{\mathrm{X}}^{\mathrm{J}}\right)\right)
\end{array}\right\}
$$

Figure 15: System of equations $E Q S$ where $X \in\{A, B\}$ and the operations mentioned should be instantiated following their definitions in the two algebras (as was exemplified after Figure 11).

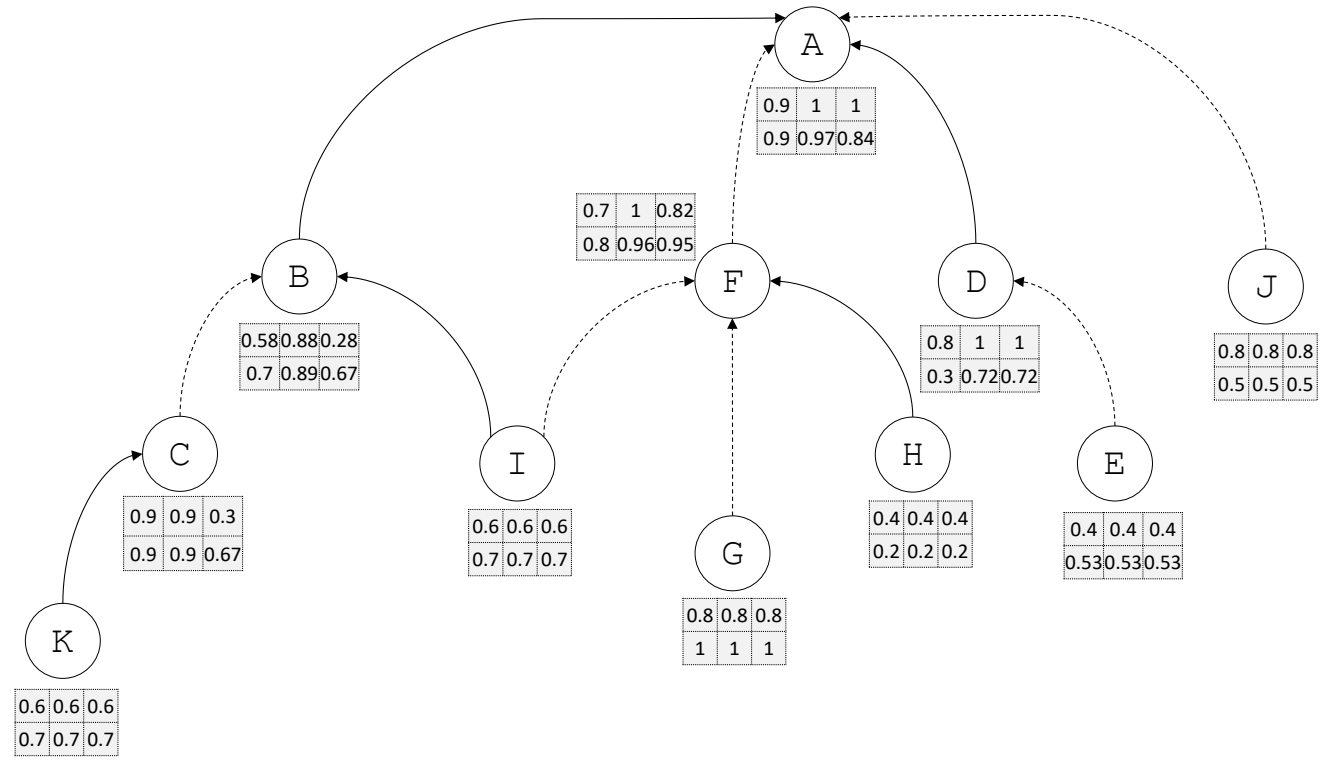

Figure 16: Final labeled bipolar argumentation graph.

Then, we classify arguments in AR according to Definition 13 as follows:

$$
\begin{aligned}
& \mathrm{AR}_{n}=\{\} \text { (neutralized), } \\
& \mathrm{AR}_{w}=\{\mathrm{A}, \mathrm{C}, \mathrm{B}\} \text { (weakened), } \\
& \mathrm{AR}_{s}=\{\mathrm{D}, \mathrm{F}\} \text { (strengthened), and } \\
& \mathrm{AR}_{u}=\{\mathrm{K}, \mathrm{I}, \mathrm{G}, \mathrm{H}, \mathrm{E}, \mathrm{J}\} \text { (unchallenged). }
\end{aligned}
$$

Next, we apply Definition 15 to obtain acceptability extensions specifying sets of acceptable arguments: $\mathrm{AR}_{w}=\{\mathrm{A}, \mathrm{B}, \mathrm{C}\}$ is a CF-W (B attacks $\mathrm{A}$; however, $\mathrm{B} \nsucc \mathrm{A}$ ) and $\mathrm{S}-\mathrm{W}$ extension, since it is 


\begin{tabular}{|c||c|}
\hline \hline Classical Extensions & Value-based Extensions \\
\hline \hline \multirow{2}{*}{ d-Preferred } & CF-W \\
& CF-U \\
& CF-S \\
\hline \multirow{3}{*}{ s-Preferred } & S-W \\
& S-U \\
& S-S \\
\hline \multirow{2}{*}{ c-Preferred } & C-W \\
& C-U \\
& C-S \\
\hline
\end{tabular}

Table 7: Comparison between Classical and Value-based extensions; greyed cells correspond to extensions obtained in our example.

a conflict-free and safe set that defends all its elements; however, it is not closed under $\mathcal{R}_{s}$ because C supports $\mathrm{B}$, which does not belong to $\mathrm{AR}_{w}$. On the other hand, set $\mathrm{AR}_{u}=\{\mathrm{K}, \mathrm{I}, \mathrm{G}, \mathrm{H}, \mathrm{E}, \mathrm{J}\}$ is a CF-U and S-U extension (H attacks F, which is supported by G; however, $\mathrm{H} \nsucc \mathrm{F}$ ), but it is not C-U since $\mathrm{G}$ supports $\mathrm{F}$, which does not belong to $\mathrm{AR}_{u}$ (the closure condition is not satisfied). The set $\mathrm{AR}_{s}=\{\mathrm{D}, \mathrm{F}\}$ is a CF-S, S-S, and C-S extension since it is conflict-free, safe and closed under $\mathcal{R}_{s}$. Also, in $\mathrm{AR}_{s}$ the argument $\mathrm{D}$ attacks $\mathrm{A}$; however, in this case $\mathrm{D} \nsucc \mathrm{A}$ following the preference function presented previously. Thus, based on this, $\mathrm{AR}_{s}$ is safe since $\mathrm{F}$ supports $\mathrm{A}$, which is attacked by $\mathrm{D}$.

In summary, Table 7 depicts the results of our approach comparing them with the classical semantic analysis. Interestingly, under the classical acceptability process we only obtain the unique d-preferred extension $S=\{\mathrm{E}, \mathrm{H}, \mathrm{I}, \mathrm{J}, \mathrm{K}, \mathrm{D}, \mathrm{G}\}$, while through our acceptability process we obtain the CF-W and S-W extension $\mathrm{AR}_{w}=\{\mathrm{A}, \mathrm{B}, \mathrm{C}\}$ considering weakened arguments, the CF-U and S-U extension $\mathrm{AR}_{u}=\{\mathrm{K}, \mathrm{I}, \mathrm{G}, \mathrm{H}, \mathrm{E}, \mathrm{J}\}$ examining unchallenged arguments, and CF-S and C-S extension $\mathrm{AR}_{s}=\{\mathrm{D}, \mathrm{F}\}$ analyzing strengthened arguments. Contrasting with our formalism, we observe that analyzing the example under the classical perspective we do not obtain any safe nor closed under $\mathcal{R}_{s}$ extensions (except for the trivial ones).

Finally, coming back to the original decision support problem, we can conclude that "Ailen Education" is a good school based on the arguments: A (weakened), F (strengthened), and G, H, I, J, and $\mathrm{K}$ (unchallenged). However, there exist counterarguments affecting this decision that were not strong enough to make the choice of this school persuasive, but that should be taken into account for further analysis: B, C and D (strengthened), and E (unchallenged).

As we can see, the additional capabilities our approach offers will provide for a more flexible and personalized analysis of the information that is available. Given the rich and varied nature of data sources, e.g., all the information that originates in social media platforms, we consider this kind of machinery to be fundamental in supporting decision-making processes by avoiding information overload, allowing the handling of personal views, and beginning to tackle malicious activities such as the publication and propagation of fake news and other related problems.

\section{Related Work}

As discussed in the introduction, rich features associated with knowledge are an essential aspect of real-world reasoning. Thus, their consideration becomes highly relevant when modeling the argumentation capabilities in intelligent systems. Next, we discuss relevant works that share this 
motivation, drawing attention to significant differences that exist with the formalism proposed in this paper.

\subsection{Interpretation of Support in Argumentation Frameworks}

Cayrol and Lagasquie-Schiex (2013) consider three recent proposals for specializing the support relation in abstract argumentation: deductive, necessary, and evidential support. Each specialization can be associated with appropriate modeling using an appropriate complex attack. Nouioua and Risch (2010) present a Dung abstract argumentation theory generalization that includes a particular kind of support relation that captures knowledge of the form "argument $a$ is necessary to obtain argument $b$ " in addition to the attack relation. In particular, the authors argue that-unlike unspecified support - the necessity relation has the advantage of ensuring that its interaction with direct attacks generates new indirect attacks having precisely the same nature of the direct ones. Boella et al. (2010) studied, in the bipolar argumentation domain (Amgoud et al., 2008), the differences between deductive support (which means that argument $a$ supports argument $b$ if the acceptance of $a$ implies the acceptance of $b$ ) and defeasible support (which means that the implication holds only by default and it can be attacked). They also analyze the loss of admissibility exposed in the classical bipolar framework, solving this problem by introducing second-order attacks not only on the attack relation but also on the support relations. Cohen et al. (2018) studied the notion of support between arguments in the context of structured argumentation systems where the elements from which arguments are composed play a crucial role. Different forms of support are presented and studied; each considers the structure of arguments and the relationships between these forms of support.

In our work, a general notion of support is taken into account, seeking to develop a formalism that is as general as possible. Encoding different interpretations, such as those described above, requires several adjustments regarding how attacks are modeled and how labels are propagated. Furthermore, such adjustments also require a re-analysis of the postulates associated with the resulting propagations. For this reason, the representation of argumentative substructures through a particular interpretation of the support relationship at an abstract level will be considered in future works, also as part of an effort to address the treatment of conflict cycles and studying mappings from our formalism to argumentation systems.

\subsection{Labeled Systems in Argumentation}

The groundbreaking work by Gabbay on Labeled Deductive Systems (Gabbay, 1993, 1996) has provided a clear and direct motivation for our work. The introduction of a flexible and rigorous formalism to tackle complex problems using logical frameworks that include labeled deduction capabilities has allowed addressing research problems in temporal logics, database query languages, and defeasible reasoning systems. In labeled deduction, the formulas are replaced by labeled formulas, expressed as $L: \phi$, where $L$ represents a label associated with the logical formula $\phi$. Labels are used to carry additional information that enriches the representation language. The intuitions attached to labels may vary according to the specific needs. The idea of structuring labels as an algebra was present from the very inception of labeled systems (Gabbay, 1993).

Gabbay's proposal was applied to argumentation systems (Gabbay, 1996) by proposing a framework with the primary purpose of formally characterizing and comparing different argument-based inference mechanisms through a unified framework; in particular, two non-monotonic inference operators were used to model argument construction and dialectical analysis in the form of war- 
rant. Labels were used in the framework to represent arguments and dialectical trees. Our proposal shares with those works the characteristic of also involving the use of labels together with an algebra. Nevertheless, our intention is focused on pursuing a different goal-we are not trying to unify the presentation of different logics and formally compare them, but to extend the representational capabilities of argumentation frameworks by allowing them to handle additional domain-specific information. It can be argued indeed that due to the extreme generality of Gabbay's framework, it could also be instantiated in some way to achieve this purpose, but we have aimed in our proposal to provide an abstract framework, showing how to propagate labels in the specific case of the argument interactions of support and conflict.

Later, concerned with a different problem, Gabbay (2012) proposed a numerical approach to the problem of merging of argumentation networks; he considers an augmented network containing the arguments and attacks of all the networks to be merged. Then, agents put forward their vote on the components of the network depending on how they perceive these components locally, where a vote means reinforcement in the sense that the more a component appears locally, the more it is represented globally. Also, he presents a way to calculate the values of arguments in the weighted augmented network and discern how the attacks to an argument affect its initial support value; finally, he presents a threshold for acceptance to determine an argument's acceptability based on its weight. Here, we focus on arguments with different roles and how the relationship between them affects acceptability; thus, our proposal shares the idea of assigning valuations to arguments and propagating these valuations through an argumentation graph. However, we allow to associate more than one attribute to the arguments and use an abstract algebraic structure in which operations of support and conflict of arguments can be performed depending on the existing relationships between them. The conflict operator models situations in which an undefeated argument is weakened when counter-arguments exist; we used the valuation associated with arguments for determining when an argument is better than another and to specify a concrete acceptability category (neutralized, weakened, strengthened, and unchallenged).

Next, we analyze several works that use particular valuations associated with arguments to specify their strength and analyze how these valuations affect the argumentative process. We discriminate our analysis by the representation capability of the formalism.

\subsection{Using Single Argument Valuations}

When studying the conceptual underpinnings of argumentative reasoning, Bench-Capon (2002) has noted that in real-life situations where arguments are in conflict, it is impossible to demonstrate which is wrong conclusively; thus, in such cases, the primary purpose of argumentative reasoning is to persuade rather than to refute. Quoting his statement in the cited paper: "The point is that in many contexts the soundness of an argument is not the only consideration: arguments also have a force which derives from the value they advance or protect.". Based on this intuition, the author proposes a formalism, called Valued-Based Argumentation Frameworks (VAF), extending Dung's model to consider the strength of arguments and, through these assessments, reflecting the preference of the audience to which the arguments are directed. Specifically, in $V A F$, an argument is associated with a value from some set that has an ordering based on a specific audience. Then, from the valuations assigned to the arguments and the preferences, it is possible to specify when an argument is strong enough to attack and defeat another. Therefore, different audiences specify different orders over the set of values, determining different defeat relations between arguments. Finally, the au- 
thors also propose a set of semantics that extend the classical ones, defining two kinds of acceptable sets: one considering those arguments accepted by all the audiences (arguments accepted objectively), and another containing those arguments that are accepted by at least one audience (those accepted subjectively). Likewise, Pollock (2010) accurately observed that in defeasible reasoning most semantical analyses disregard the matter of the inner force of arguments; that is to say that in a real debate some arguments support their conclusions with more force than others. However, the recognition that arguments can differ in strength, and therefore, their conclusions might differ in their degree of justification, leads to an increase in the complexity of the analysis. Thus, Pollock proposes the addition of a type of argument that he calls diminishers, i.e., those that cannot wholly defeat their target, but they are able to lower the degree of justification of the argument over which they perform the attack. These ideas provided motivation for our work, analyzing the requirement that features associated with the arguments (independently of their required logical soundness) are essential when an argumentation discussion is analyzed. This necessity becomes even more critical in the domain of social networks, where it is crucial to take into account different issues. Specifically, based on the intuitions introduced in these research lines, we combine Bipolar Argumentation Frameworks with Algebras of Argumentation Labels to extend the representation capability of argument structures; in this system, labels represent argument features, generalizing the notion of value and weight and allowing for multiple aspects being represented. Moreover, we recognize the fact that the interaction between arguments can affect their labels, causing strengthening and weakening among arguments. Thus, the information contained in the labels enables us to improve the analysis performed over the argumentation framework and refine it limiting this analysis just to the set of relevant arguments. In particular, in this expanded framework, it is possible to determine the acceptability of sets of arguments, as well as additional information justifying their acceptability status.

The ability of the abstract framework proposed by Dung to analyze and treat the inconsistency associated with a knowledge base is well known; this framework identifies the arguments that describe a specific discussion and establishes a defeat relation between conflicting arguments. Then, through a collective semantic process, it analyzes the discussion to determine the acceptability status (accepted or rejected) associated with the involved arguments. However, due to this formalism's high-level abstraction, it is not possible to analyze the arguments' acceptability from an individual perspective since their individual properties are unknown. Amgoud and Cayrol (1998) proposed a formalism, called Preference-Based Argumentation Frameworks, where a preference relation between the arguments is introduced in the classical abstract argumentation framework in order to consider the user's preferences. The defeat relation represents a conflict based on purely logical properties (such as "rebut" or "undercut"), while the preference relation represents the preferences (meta-knowledge) that cannot be extracted from the arguments themselves. Then, using a combination of these relations, it is possible to conclude that an argument defends itself when it is preferred over all its attackers, or an argument is defended when there exists a set of arguments that defeats its attackers, being the arguments of the defending set preferred to the arguments of the attacking set. In contrast, our formalism details the arguments' features used as a tool to specify preferences between arguments to establish some properties associated with a specific set of arguments. Thus, the preference function is not used in the conflict resolution process, since this role is performed by the conflict operator defined in the algebra of labels, which specifies how the valuations of arguments involved in a conflict relation are affected. 
Elvang-Gøransson et al. (1993) analyze the fact that non-trivial arguments may be constructed for and against a specific proposition in the presence of an inconsistent database; the problem arises when determining which conclusion must be accepted. The authors define a particular concept of acceptability, which is used to reflect the different acceptability levels associated with an argument; then, they argue that "the more acceptable an argument, the more confident we are in it". Additionally, they define acceptability classes to assign linguistic qualifiers to the arguments. There are some similarities between this proposal and our own, starting from the fact that the relationships between the arguments are analyzed, and the acceptability class they belong to is determined. However, they do not take into account the domain-dependent characteristics associated with the arguments, only a single value is considered to determine argument strength, and they do not analyze the user's perspectives. The authors also work over a structured argumentation system while we work at an abstract level.

Potyka (2018) proposes a continuous dynamical system as a well-suited tool to analyze cyclic and acyclic bipolar argumentation frameworks, arriving at a convergence state. Towards this end, the author gives the conditions under successive procedures that can be transformed into well-defined dynamical systems; furthermore, the model satisfies a set of axiomatic properties that complement the existing approaches. In contrast with our work, this approach includes the possibility of dealing with cyclic bipolar argumentation frameworks; however, the author proposed a special and unique propagating function, where the valuations given by this function may not always represent realworld behavior. In our formalism, the acyclic bipolar argumentation framework is treated by a labeling procedure, where the operators that propagate the argument features can be instantiated in different ways, depending on the real-world domain. Moreover, we analyze the set of models that satisfy objective functions introduced by the user to model a user position that maximizes, or minimizes, specific argument features. In addition, in A, we present a pruning process to optimize the argumentation framework by analyzing the influence of the arguments involved in the discussion.

Cayrol and Lagasquie-Schiex (2005a) describe a two-step argumentation process: (i) the calculation of a valuation of the relative strength of the arguments, and (ii) the selection of the most acceptable among them. Their focus is on defining a gradual valuation of arguments based on their interactions and establishing a graded concept of acceptability. The authors assert that an argument is all the more acceptable if it can be preferred to its attackers and propose a domain of argument valuations where aggregation and reduction operators are defined; however, the arguments' evaluation is solely based on their interaction. In our work, we determine valuations through users' perceptions, considering the different interactions among them, and propagating the valuations using the operations defined in the algebra of labels. Unlike the proposal of Cayrol and LagasquieSchiex, the operations assigned to each relation among arguments are defined by the user-this provides the possibility of explicitly considering the problem's domain. Moreover, we provide the ability to assign more than one valuation to the arguments, depending on the features we wish to model. Our formalism also gives the possibility to obtain a feasible solution based on user perception that optimizes some measure under uncertainty. Finally, after analyzing all the interactions among arguments, we obtain final valuations assigned to each argument; then, through these valuations, the acceptability status (strengthened, unchallenged, weakened, or neutralized) of arguments is obtained.

Rago et al. (2016b) propose an algorithm computing only acyclic graphs for quantifying the strength of alternative decision options, based on the aggregation of the strength of their attacking and supporting arguments, proving a set of desirable properties that highlights the usefulness 
and proper propagation of values in the debate domain. In the same direction, the authors define a quantitative semantics (Rago, Čyras, \& Toni, 2016a) (adapting the algorithm proposed in the previous work) for evaluating the strength of arguments in bipolar argumentation frameworks, studying the relationship between the new semantics and some existing semantics for other argumentation frameworks, as well as some properties of a specific semantics. Furthermore, under certain constraints, they can compute cyclic graphs in the bipolar domain (assuming that all arguments have a base score of 0.5). However, in our work, the algebra of labels allows us to perform a more general propagation without conforming to specific functions, supporting different propagations according to each attribute's nature. A wide range of properties is provided, verifying the labeling process has an adequate behavior and robustness, obtaining a set of semantics useful in the argumentative domain. Also, only acyclic graphs are treated; in Appendix B we present and discuss several ideas to address graphs with conflict cycles without loss of generality.

\subsection{Using Multiple Argument Valuations}

Kaci et al. (2008) generalized Bench-Capon's value-based argumentation frameworks in such a way that arguments can promote multiple values, and preferences among values or arguments can be specified in various ways. Each value can be associated with one or more arguments and vice versa; then, once the different values are mapped to each argument involved in the discussion, the existing conflict relations are analyzed to identify the successful attacks. In Bench-Capon's value-based framework, the attack of an argument A over an argument B is successful if and only if A attacks B and the value promoted by B is not preferred over that promoted by A; however, in this new proposal, arguments can promote more than one value; this increases the difficulty of determining when an argument is preferred to another based on their valuations. To address this problem, the authors provide two guidelines, based on the principles of minimal/maximal specificity, that allow establishing a unique possible ordering (total order) over the set of values associated with the arguments. Then, based on this order, it is possible to obtain the successful attacks and subsequently, the acceptable arguments; this is done by combining algorithms from non-monotonic reasoning with others for calculating extensions in abstract argumentation. The main idea in that approach has certain similarities with our work: the valuations associated with the arguments provide the possibility of establishing argument strength, and the arguments have associated different valuations representing attributes that are not related to the logical soundness of the arguments. However, from our perspective, each of the features associated with the arguments must have a particular interpretation, a particular ordering, and individual treatment. Towards this end, we have incorporated a set of algebras of argumentation labels that are provided to represent and compute the different characteristics of the arguments modeling the knowledge behavior in the argumentation domain.

Motivated by the idea of encouraging and enhancing a debate on a particular topic in social media, Leite et al. (2011) proposed an extension of Dung's framework with the possibility to associate votes to arguments, together with a semantics that assigns a value to each argument; such values are drawn from a predetermined set of possible values, and represent the arguments' strength (taking into account both the structure of the graph and the social opinion expressed through the votes). This proposal has some similarities with the one presented in this paper since both obtain additional information regarding the quality of the arguments. However, our formalism can be interpreted as a generalization of the social argumentative framework allowing to represent multiple features associated with arguments, such as user preferences and the accuracy of the information 
that the arguments represent, among others. Furthermore, our formalism allows us to represent positive (support) and negative (attack) relations between arguments, while the social argumentation framework only represents an attack between contradictory arguments. On the other hand, some similarities can be found between the operations used to manipulate the social strength of the arguments and the operations defined in the algebra of argumentation labels; however, conflict resolution is modeled differently.

In a series of works, Amgoud et al. $(2016,2018,2019)$ introduce a closely related line of research, where the authors define principles that a particular semantics would satisfy in a bipolar setting. Such principles are useful for defining reasonable semantics, for a better understanding of the design choices or foundations of each semantics, and for comparing pairs of semantics. Furthermore, the authors propose the definition of a novel gradual semantics for the sub-class of nonmaximal acyclic bipolar graphs, showing that it satisfies a set of principles. Our formalism shares the same goal, with particular attention over a set of well-defined operators that propagate the features in an argumentative discussion. These operators can be instantiated in different ways, each of them giving place to a specific semantics that models the constraints of the domain. Moreover, we can refine the argumentative discussion introducing objective functions that specify the user's intentions to optimize a specific viewpoint, shifting the model from one perspective to another. On the other hand, we present a set of tools to improve the discussion, analyzing the effectiveness of the support and conflict relations, and the quality associated with the participating arguments. Additionally, we present an algorithm that implements the labeling process with tractable computational complexity in acyclic argumentation graphs.

\subsection{Fuzzy and Probabilistic Frameworks}

There have been many developments centered around the extension of argumentation frameworks with machinery for representing and reasoning with fuzzy and probabilistic information.

Several works discuss using fuzzy sets and relations to refine Dung's semantics, as we do here in the bipolar argumentation domain. In particular, Janssen et al. (2008) propose fuzzy argumentation frameworks as a conservative extension of traditional Dung argumentation frameworks. The fuzzy approach enriches the classical argumentation model's expressive power by allowing to represent the relative strength of the attack relationships between arguments and the degree to which arguments are accepted. In the same direction, Gratie and Florea (2014) introduce a formalism where fuzzy labels are used in Dung's abstract argumentation. The first approach we propose is built as a natural extension of the \{in, out, undecided $\}$ labeling to real-valued labels, coupled with an unsupervised learning algorithm that assigns consistent labels starting from a random initial assignment. The second approach regards argument (fuzzy) labels as degrees of certainty in the argument's acceptability; this translates into a system of equations that provides among its solutions the labelings that describe complete extensions. The latter's main innovations are that we allow for flexible use of different operations, introducing the conflict process to weaken arguments and the support operation to strengthen them. Also, after analyzing all the interactions among arguments, the acceptability status (strengthened, unchallenged, weakened, or neutralized) is obtained. Our formalism also gives the possibility to obtain a feasible solution under a user's perception that optimizes some measure under uncertainty.

In another direction, there exist works where probabilistic notions are explored in the argumentation domain, extending abstract models. Hunter and Thimm (2017) explain that abstract argu- 
mentation can be enhanced by considering probability assignments on arguments, allowing for a quantitative treatment of formal argumentation. In particular, the epistemic approach analyzes the topology of the graph where probabilistic assessments on the acceptance of arguments are evaluated w.r.t. the relations of the arguments in the graph. The core idea is that the more likely one is to believe in an argument, the less likely one is to believe in an argument attacking it. The epistemic approach is useful for modeling the beliefs that an opponent might have in the arguments presented, which is useful, for example, when deciding on the best arguments to present to persuade an opponent. Thus, under this proposal, they assign to each argument a degree of belief under a specific probability function, affecting the argumentation graph where it plays a particular role. That is, an agent may disbelieve some of the arguments based on what it knows about the topic. Furthermore, it may disbelieve some of the arguments that are not attacked. Finally, some constraints on the probability function can be considered, which may take different aspects of the argument graph structure into account. Furthermore, in a related research line, Fazzinga et al. (2015) analyze the combination of Dung's abstract argumentation framework with probability theory to model uncertainty in argumentation. In this setting, they address the fundamental problem of computing the probability that a set of arguments is an extension according to a given semantics. That is, they characterize the computational complexity of associating a probability distribution over the set of arguments, and the complexity of obtaining specific semantics.

Our work is closest in spirit to the latter since labels can be seen as a generalization of probability values associated with arguments; however, it is not complete subsumption since the algebra, in this case, would need to model a probabilistic space; the modeling of the corresponding probability distribution needs to reside elsewhere, as done in the works mentioned above. Furthermore, the spirit of the values associated with the arguments is primarily to see how they are affected by interactions within the argumentative model; that is, modeling the behavior of knowledge within the discussion. In this sense, probabilistic values would not have to be modified by the existence of an attack, but rather the probability should be a parameter to consider or not to consider such attacks. Of course, in the absence of attacking arguments, the probability of the arguments attacked may rise, but they still have different interpretations.

Hunter et al. (2020) introduce epistemic graphs as a generalization of the epistemic approach to probabilistic argumentation. In these graphs, an argument can be believed or disbelieved up to a given degree, thus providing a more fine-grained alternative to the standard Dung-based approaches when it comes to determining the status of a given argument. Furthermore, the flexibility of the epistemic approach allows both formalisms to model the rationale behind the existing semantics and completely deviate from them when required. The interesting issue is that they analyze epistemic graphs that can model both attack and support and relations that are neither support nor attack. The way other arguments influence a given argument is expressed by the epistemic constraints that can restrict the belief that they have about an argument with a varying degree of specificity. The fact that they can specify the rules under which arguments should be evaluated, and they can include constraints between unrelated arguments permits the framework to be more context-sensitive. As mentioned above, the nature of the values in our work is different from the more specific probability values, and some adjustments are required to handle them in our algebra. Thus, this is part of future work and the definition of an algebra that can handle temporal notions. 


\begin{tabular}{|c|c|c|c|c|c|c|c|c|c|c|}
\hline & $\begin{array}{c}\text { (1) } \\
\text { Multiple } \\
\text { Values }\end{array}$ & $\begin{array}{c}(2) \\
\text { General } \\
\text { Propagation }\end{array}$ & $\begin{array}{c}(3) \\
\text { Uncertainty } \\
\text { Ranges }\end{array}$ & $\begin{array}{c}(4) \\
\text { Domain- } \\
\text { dependent } \\
\text { Features }\end{array}$ & $\begin{array}{c}(5) \\
\text { Bipolarity } \\
\text { (Pos/Neg } \\
\text { Relations) }\end{array}$ & $\begin{array}{c}(6) \\
\text { Weakening } \\
\text { Relation }\end{array}$ & $\begin{array}{l}(7) \\
\text { Treatment of } \\
\text { Cycles }\end{array}$ & $\begin{array}{c}\text { (8) } \\
\text { Level of } \\
\text { Abstraction }\end{array}$ & $\begin{array}{c}\text { (9) } \\
\text { Solution } \\
\text { Space } \\
\text { Optimiz. }\end{array}$ & $\begin{array}{c}\text { (10) } \\
\text { Categories of } \\
\text { Acceptable } \\
\text { Arguments }\end{array}$ \\
\hline $\begin{array}{l}\text { Valued-based } \\
\text { Argumentation } \\
\text { (Bench-Capon) }\end{array}$ & $x$ & $x$ & $x$ & $x$ & $x$ & $x$ & $\checkmark$ & ABSTRACT & $x$ & $x$ \\
\hline $\begin{array}{l}\text { Argument-based } \\
\text { Defeasible Reasoning } \\
\text { (Pollock) }\end{array}$ & $x$ & $\checkmark$ & $x$ & $x$ & $x$ & $\checkmark$ & $\checkmark$ & ABSTRACT & $x$ & $\times$ \\
\hline $\begin{array}{l}\text { Preference-based } \\
\text { Argumentation } \\
\text { (Amgoud and Cayrol) }\end{array}$ & $x$ & $x$ & $x$ & $x$ & $x$ & $x$ & $\sqrt{ }$ & ABSTRACT & $x$ & $x$ \\
\hline $\begin{array}{l}\text { Dialectical Reasoning } \\
\text { (Elvang-Gøransson) }\end{array}$ & $x$ & $x$ & $x$ & $x$ & $x$ & $x$ & $\sqrt{ }$ & STRUCTURED & $x$ & $x$ \\
\hline $\begin{array}{l}\text { Continuous Dynamical } \\
\text { Systems (Potyka) }\end{array}$ & $x$ & $x$ & $x$ & $x$ & $x$ & $x$ & $\checkmark$ & STRUCTURED & $x$ & $\checkmark$ \\
\hline $\begin{array}{l}\text { Graduality in } \\
\text { Argumentation } \\
\text { (Cayrol and Lagasquie-Schiex) }\end{array}$ & $x$ & $\checkmark$ & $x$ & $x$ & $\checkmark$ & $x$ & $\checkmark$ & ABSTRACT & $x$ & $x$ \\
\hline $\begin{array}{l}\text { Multiple Value-based } \\
\text { Argumentation (Kaci et al.) }\end{array}$ & $\checkmark$ & $x$ & $x$ & $x$ & $x$ & $x$ & $\checkmark$ & ABSTRACT & $x$ & $x$ \\
\hline $\begin{array}{l}\text { Social Argumentation } \\
\text { (Leite et al.) }\end{array}$ & $\times$ & $\sqrt{ }$ & $x$ & $\checkmark$ & $x$ & $\times$ & $\checkmark$ & ABSTRACT & $\times$ & $\times$ \\
\hline $\begin{array}{l}\text { Labeled Bipolar } \\
\text { Argumentation } \\
\text { (This work) }\end{array}$ & $\checkmark$ & $\checkmark$ & $\checkmark$ & $\checkmark$ & $\checkmark$ & $\checkmark$ & $\begin{array}{c}\text { PRELIMINARY } \\
\text { Results }\end{array}$ & ABSTRACT & $\checkmark$ & $\checkmark$ \\
\hline
\end{tabular}

Figure 17: Comparing Argumentation Frameworks Tools.

\subsection{Summary of Related Approaches}

To summarize the wide variety of approaches we discussed above, in Table 17 we present an analysis based on the main features that characterize them, making it easier to compare specific ones in terms of their capabilities: (1) Multiple values: the formalism can represent and manipulate more than one attribute value simultaneously; (2) General propagation: support for instantiation of different propagation operators according to the domain's constraints; (3) Uncertainty ranges: second-order uncertainty (intervals instead of single values); (4) Domain-dependent Features: support for features that go beyond interaction between arguments; (5) Bipolarity: availability of both positive (support) and negative (attack) relations; (6) Weakening relation: support for finer-grained analyses of conflicts between arguments; (7) Treatment of cycles: support for cyclic relations; (8) Level of abstraction: abstract vs. structured arguments; (9) Solution space optimization: availability of tools to reduce the solution space towards simpler analyses and/or lower computational costs; and (10) Categories of acceptable arguments: categorization of accepted arguments beyond the consideration of graduation levels (as commonly used in argumentative systems based on argument ranking).

As can be seen in the table, an important issue that needs to be addressed in our work is the treatment of conflict cycles. In this paper, we show preliminary results in this direction, which are presented in Appendix B. Solving this non-trivial problem is the topic of ongoing and future work.

\section{Conclusions and Future Work}

Depending on the application, it is sometimes necessary to associate additional information with arguments to accurately represent their features - the goal is to leverage this information in determining their acceptability status. For instance, in a multi-agent scenario, one might establish a relevance degree associated with the arguments representing the agents' preferences, consider the 
popularity of an argument based on the social vote associated with it, among others. Here, we developed the Labeled Bipolar Argumentation Framework ( $L-B A F)$, which combines the representation capabilities provided by Bipolar Argumentation Frameworks $(B A F)$ and the manipulation of additional labels using an algebra developed for this purpose; in that way, interactions between arguments have associated operations defined over the algebra, allowing information to be propagated. In this expanded framework, it is feasible to determine the acceptability of sets of arguments as well as additional information that justifies their acceptability status. This possibility means that our formalism allows a more granulated analysis of the arguments and their relationships, representing the effect (positive and negative) of one argument over others; then, we use the final features associated with each argument to classify them, and finally, over each relevant class, we looked for some critical properties such as conflict freeness, safety, and closure under support. Also, we presented a pruning process to optimize the argumentation framework by analyzing the influence of the arguments involved in the discussion. Thus, a set of coefficients representing the quality of an argumentation framework is extracted and used to dismiss arguments from consideration or to drop relationships between them.

Current and future work involves developing an implementation of $L-B A F$ by instantiating it in the existing DeLP (García \& Simari, 2014) system as a basis; the resulting implementation will be evaluated in different domains that require extra information associated with arguments, taking as motivation studies and analyses such as P-DeLP (Alsinet et al., 2008a, 2008b). In another direction, we will deepen the study of the bipolar cyclic argumentative frameworks and their treatment in the proposed formalism. Furthermore, we are interested in analyzing the computational complexity associated with the proposed formalism and how it is affected by the operators defined in the algebra and the number of arguments interacting in a specific scenario.

\section{Acknowledgments}

This work has been partially supported by EU H2020 research and innovation programme under the Marie Sklodowska-Curie grant agreement No. 690974 for the project MIREL: MIning and REasoning with Legal texts, and by funds provided by CONICET, Institute for Computer Science and engineering (ICIC UNS-CONICET), Universidad Nacional del Sur (UNS), Agencia Nacional de Promoción Científica y Tecnológica, and Universidad Nacional de Santiago del Estero, Argentina.

\section{Appendix A. Refining an Argumentative Discussion}

In any discussion, arguments proclaim assertions of different kinds; it would be interesting to have the capability to analyze a scenario considering all arguments that have specific features suitable for the topics being addressed. In this sense, in real-world applications, it can be useful to refine argumentative frameworks by considering only those arguments that satisfy specific constraints relevant to a given domain. For example, an agent needing to make a decision with a high degree of reliability by considering only the best arguments involved in a discussion, or an agent making a decision based only on the arguments with a specific social rating. In this direction, having extra information associated with arguments introduces the possibility of improving the argumentative discussion by excluding the least relevant arguments.

Different research lines have addressed the analysis of different aspects of an argumentation framework to optimize it, applying specific techniques. In this direction, Bonzon et al. (2014) ad- 
dress the uncertainty faced by a user participating in multiagent debate. They propose a way to compute the relative relevance of arguments for such a user by merging the classical argumentation framework into a game-theoretic coalitional setting, where the worth of a collection of arguments (opinions) can be seen as the combination of the information concerning the defeat relation and the preferences over arguments of a user. Via a property-driven approach, they show that the Shapley value for coalitional games defined over an argumentation framework can be applied to summarize all the information about the worth of opinions into an attribution of relevance for the single arguments. Furthermore, Bistarelli and Taticcihi (2019) explain that cooperative games are a class of games where groups of players (or agents) compete to maximize their goal, through one or more specific rules. In particular, voting games are a category of cooperative games in which each player's contribution determines the profit of labeling associated with a specific argumentation model. Thus, to identify the value brought from a single player to a specific labeling, power indexes (Shapley value and Banzhaf power index) are used, supporting a ranking process between the arguments.

In the same research thread, but analyzing the attack contribution in a discussion, Amgoud (2017) argued that the acceptability degree of an argument should be equal to the primary strength of the argument if the latter is not attacked. Otherwise, the argument is weakened by its attackers and loses weight, leading to an acceptability degree lower than the primary strength. Hence, from the outcome of a semantics, it is possible to compute each argument's global loss because of its attackers. It is the difference between the primary strength of the argument and its acceptability degree. However, it is not possible to say anything regarding the contribution of each attack to that loss. That contribution represents, in some sense, the intensity of the attack. The greater the contribution of an attack, the more harmful the attack. Information on the contribution of attacks is very useful since it allows a better understanding of each one's impact. Namely, it allows detecting worthless attacks (i.e., attacks that do not have any impact on the target), and redundant ones (i.e., attacks that lead to the same loss for their target). This ranking is very useful, especially in persuasion dialogues, where agents have to choose the best counter-attack to win a dialogue. Thus, they propose the novel concept of contribution measure, which takes as input an argumentation framework, and returns as output a weight for each attack, representing the attack's contribution.

As in these research lines, the values associated with an argument in L-BAFs allow us to analyze the argumentation framework in three directions: (i) coefficients of attack and support, indicating the effectiveness of the relations defined among the arguments, (ii) argument quality coefficients to establish the quality of individual arguments participating in the discussion. To do this, we analyze the relationship between the original value, a strengthened value after considering the supporting arguments, and a overall value when considering the attackers and the supporters together (we can establish how important their supporters and attackers are to each of them). Finally, (iii) we can apply these coefficients using them to prune arguments to obtain a relevant, focused, and competent discussion. The following definition aims to address points $(i)$ and $(i i)$.

Definition 17 Let $\Psi=\langle\Theta, \mathbf{A}, v\rangle$ be an $L-B A F, G_{\Psi}$ be the corresponding labeled bipolar argumentation graph, $\mathbf{A}$ be the set of label algebras, $\mathbf{X} \in \mathbf{A}$ be an algebra of argumentation labels, and $\# \mathcal{R}_{a}$ and $\# \mathcal{R}_{s}$ be the cardinalities of the attack and support relations, respectively. Then, the effectiveness degree associated with the argument roles in $\Psi$ is defined as follows where in each case denominators assumed to be non-zero, otherwise the coefficient is 0: 
Particular Effectiveness Attack

$\omega_{\mathrm{X}}^{\mathrm{a}}=\frac{\sum_{\mathrm{A} \in \mathrm{AR}} \mu_{\mathrm{X}}^{\mathrm{A}}-\delta_{\mathrm{X}}^{\mathrm{A}}}{\# \mathcal{R}_{a}}$

Particular Effectiveness Support

$\omega_{\mathrm{X}}^{\mathrm{s}}=\frac{\sum_{\mathrm{A} \in \mathrm{AR}} \mu_{\mathrm{X}}^{\mathrm{A}}-\alpha_{\mathrm{X}}^{\mathrm{A}}}{\# \mathcal{R}_{s}}$

Particular Argument Quality

$\omega_{\mathrm{X}}^{\mathrm{q}}=\frac{\sum_{\mathrm{A} \in \mathrm{AR}} \alpha_{\mathrm{X}}^{\mathrm{A}}}{\# \mathrm{AR}}$
General Effectiveness Attack

$\Omega_{\Psi}^{\mathrm{a}}=\frac{\sum_{\mathbf{X} \in \mathbf{A}} \omega_{\mathrm{X}}^{\mathrm{a}}}{\# \mathbf{A}}$

General Effectiveness Support

$\Omega_{\Psi}^{\mathbf{s}}=\frac{\sum_{\mathbf{X} \in \mathbf{A}} \omega_{\mathbf{X}}^{\mathbf{s}}}{\# \mathbf{A}}$

General Argument Quality

$\Omega_{\Psi}^{\mathrm{q}}=\frac{\sum_{\mathbf{X} \in \mathbf{A}} \omega_{\mathrm{X}}^{\mathrm{q}}}{\# \mathbf{A}}$

Example 6 Returning to Example 4, the particular conflict, support, and argument quality coefficients are: $\omega_{\mathrm{A}}^{\mathrm{a}}=0.18, \omega_{\mathrm{A}}^{\mathrm{s}}=0.14$, and $\omega_{\mathrm{A}}^{\mathrm{q}}=0.56$ (w.r.t. accuracy) and $\omega_{\mathrm{B}}^{\mathrm{a}}=0.29, \omega_{\mathrm{B}}^{\mathrm{s}}=0.22$, and $\omega_{\mathrm{B}}^{\mathrm{q}}=0.58$ (w.r.t. reliability); the general conflict, support, and argument quality coefficients are: $\Omega^{\mathrm{a}}=0.24, \Omega^{\mathrm{s}}=0.18$, and $\Omega^{\mathrm{q}}=0.57$.

In a general sense, the attack and support coefficients establish that the arguments' effectiveness is quite low, while the quality of the arguments involved in the argumentation discussion is around an intermediate value.

The following proposition establishes a relation between the coefficients of support and quality. Briefly speaking, when the quality associated with the arguments is 1 (the highest value), the support effects between them is irrelevant; this means that these arguments can be taken as truths tentatively, for this reason, they do not need support that justifies their validity.

Proposition 6 Let $\Psi=\langle\Theta, \mathbf{A}, v\rangle$ be an $L-B A F$, and $\Omega_{\Psi}^{\mathrm{q}}$ and $\Omega_{\Psi}^{\mathrm{s}}$ be the general effectiveness support and quality coefficients associated with $\Psi$, respectively. We have that: if $\Omega_{\Psi}^{\mathrm{q}}=1$, then $\Omega_{\Psi}^{\mathrm{s}}=0$.

Analyzing a discussion where the arguments involved have the maximal possible quality (each feature associated with the argument has the value 1), we conclude that no weakening nor strengthening between arguments can be produced. The following proposition formally states that result.

Proposition 7 Let $\Psi=\langle\Theta, \mathbf{A}, v\rangle$ be an $L-B A F, \Omega_{\Psi}^{q}$ be the general quality coefficient associated with $\Psi$, and $\mathrm{AR}_{w}$ and $\mathrm{AR}_{s}$ be the sets of weakened and strengthened arguments associated with $\Psi$, respectively. We have that, if $\Omega_{\Psi}^{\mathrm{q}}=1$, then $\mathrm{AR}_{w}=\emptyset$ and $\mathrm{AR}_{s}=\emptyset$.

Once the arguments are labeled, we can consider their acceptability status. In this direction, we use the information attached to the arguments to provide different degrees, or rankings, of acceptability. Next, we formalize the pruning approach presented in point (iii) above.

Definition 18 Let $\Psi=\langle\Theta, \mathbf{A}, v\rangle$ be an $L-B A F, G_{\Psi}$ be the corresponding labeled bipolar argumentation graph, and $\mathbf{A}$ be the set of algebras of labels. The pruned labeled bipolar argumentation framework based on $\Psi$ is the 3-tuple $\Psi^{\prime}=\left\langle\Theta^{\prime}, \mathbf{A}, v\right\rangle$ where $\Theta^{\prime}=\left\langle\mathrm{AR}^{\prime}, \mathcal{R}_{a}^{\prime}, \mathcal{R}_{s}^{\prime}\right\rangle$ is the pruned BAF such that:

$-\mathrm{AR}^{\prime}=\left\{\mathrm{A} \in \mathrm{AR} \mid \alpha_{\mathrm{X}}^{\mathrm{A}} \geq \omega_{\mathrm{X}}^{\mathrm{q}}\right.$ for each $\left.\mathrm{X} \in \mathbf{A}\right\}$, i.e., $\mathrm{AR}^{\prime}$ is the set of relevant arguments in $\mathrm{AR}$. 
- $\mathcal{R}_{a}^{\prime}=\left\{(\mathrm{A}, \mathrm{B}) \in \mathcal{R}_{a}\right.$ with $\mathrm{A}, \mathrm{B} \in \mathrm{AR}^{\prime} \mid \delta_{\mathrm{X}}^{\mathrm{A}} \geq \omega_{\mathrm{X}}^{\mathrm{a}}$ for each $\left.\mathrm{X} \in \mathbf{A}\right\}$, i.e., $\mathcal{R}_{a}^{\prime}$ is the relevant attack relation defined over $\mathrm{AR}^{\prime}$, and

- $\mathcal{R}_{s}^{\prime}=\left\{(\mathrm{A}, \mathrm{B}) \in \mathcal{R}_{s}\right.$ with $\mathrm{A}, \mathrm{B} \in \mathrm{AR}^{\prime} \mid \delta_{\mathrm{X}}^{\mathrm{A}} \geq \omega_{\mathrm{X}}^{\mathrm{s}}$ for each $\left.\mathrm{X} \in \mathbf{A}\right\}$, i.e., $\mathcal{R}_{s}^{\prime}$ is the relevant support relation defined over $\mathrm{AR}^{\prime}$.

After applying this pruning process, it is possible to analyze its effects, captured through the following underlying principles:

Principle 2 The valuations obtained as a result of applying the pruning process given in Definition 18 meet the following principles:

- Strengthening by Support: Adding a new undefeated supporting argument can reduce the weakening effects over the strength of the argument supported. A change in strength always has a discernible cause;

- Strengthening by Removal: Removing an attacker can reduce the weakening effects over the strength of the attacked argument. As with support, changes in strength always have a discernible cause; and

- Ancestral Strengthening: The increase in strength of an argument can increase the strength of all the supported arguments. A change in the subgraph that affects the strength associated with an argument provoking its strengthening implies an increase of the strength associated with each argument that such argument supports.

First, by removing attacking arguments or an attack relation, defeated arguments may become weakened or even non-attacked arguments - these arguments thus recover their partial or total strength, respectively. Indeed, removing attacking arguments, defeated arguments can end up having a strengthened status if they are supported in the discussion: when the accumulation of the support strength exceeds the accumulation of the attack strength, it concludes in a strengthening of the affected argument. So, a side effect of this action would be the strengthening of arguments; that is, the restored arguments can strengthen supported arguments. More specifically, if we consider a non-attacked supported argument, the strength restoration of the supporting argument is propagated directly. On the other hand, if we consider an attacked supported argument, the strength restoration of the supporting argument can mitigate or neutralize that attack. Following Definition 18, the behavior associated with the bipolar model can be modified, removing arguments and relations. In Figure 18, we analyze each case: (a) presents the strengthening by support removing both the attacking arguments and the conflict relation from the discussion (the reason would be that both the quality of argument $\mathrm{C}$ and the effect that $\mathrm{C}$ has on $\mathrm{B}$ are less than the conditions required; that is, $\alpha_{\mathrm{X}}^{\mathrm{C}}<\omega_{\mathrm{X}}^{\mathrm{q}}$ and $\delta_{\mathrm{X}}^{\mathrm{C}}<\omega_{\mathrm{X}}^{\mathrm{a}}$ ), while (b) shows the strengthening by removal under the same constraints; on the other hand, (c) analyzes the ancestral strengthening removing only the conflict relation between arguments $\mathrm{C}$ and B. From now on, the arguments that do not make the cut are greyed and are thus pruned, and the corresponding edges, marked with $\times$, are eliminated.

The three following principles describe when a change in an argumentation framework can improve or degrade the ranking of an argument. These properties have been proposed informally by Cayrol and Lagasquie-Schiex (2005a) in the context of the semantics they introduced; we now analyze them in the context of our formalism. 


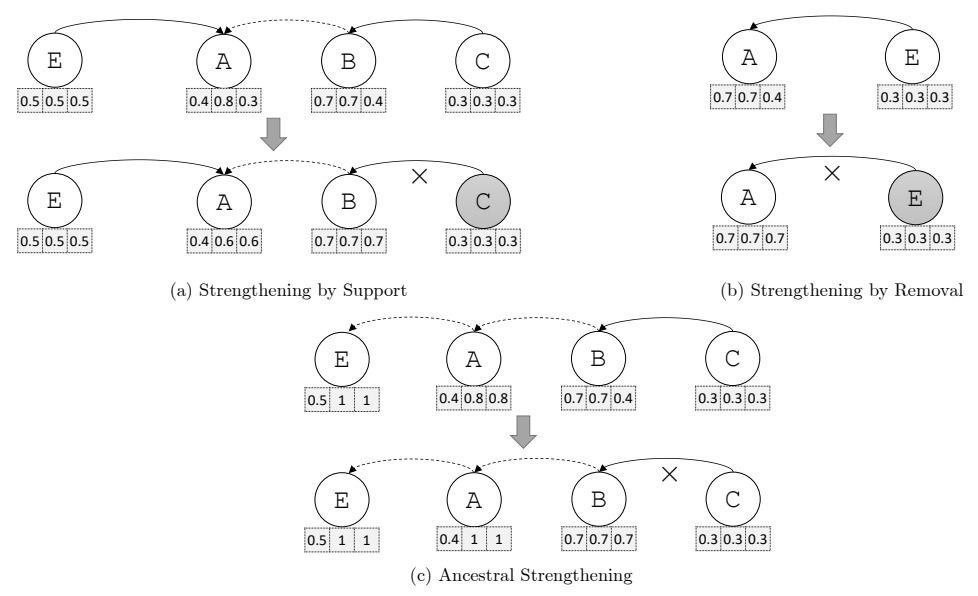

Figure 18: Strengthening in $L-B A F$.

Principle 3 The valuations given by Definition 11 after applying the pruning process established in Definition 18 abide by the following principles:

- Improvement of a Defense Branch: improving a defense branch to any argument can increase its overall valuation.

- Improvement of a Support Branch: improving a support branch to any argument can increase its strengthened valuation.

- Improvement of an Attack Branch: improving an attack branch to any argument can decrease its overall valuation.

Extending the explanation introduced before to a chain of arguments, we can analyze the following situation (cf. Figure 19). On the one hand, the positive effect is related to the improvement of support and defense branches, removing an attacking argument or an attack relation that breaks an attack branch; for example, in Figure 19(a) the defense associated with argument A is improved by removing $E$, since $E$ attacks $C$, which is the direct defense of $A$, thus weakening the strength of B. On the other hand, the negative effect produced over an argument is the improvement of an attacking branch, removing an attacking argument or an attack relation that breaks a defense branch; in Figure 19(c) the attack produced from $A$ to $E$ is improved after removing C, since $C$ was an attacker of $\mathrm{B}$, which restores its strength and thus improves the support of $\mathrm{A}$.

After applying the refining process over an argumentation discussion, the overall argument quality improves. This result is reflected in the following proposition.

Proposition 8 Let $\Psi=\langle\Theta, \mathbf{A}, v\rangle$ be an $L-B A F$ and $\Psi^{\prime}=\left\langle\Theta^{\prime}, \mathbf{A}, v\right\rangle$ be the pruned labeled bipolar argumentation framework based on $\Psi$. Then, the relation between the general quality of arguments satisfies that $\Omega_{\Psi}^{\mathrm{q}} \leq \Omega_{\Psi^{\prime \prime}}^{\mathrm{q}}$.

Example 7 Continuing with Example 6, we proceed to carry out the pruning process from Definition 18. Once the pruning process is completed, it is necessary to propagate the valuations associated with the arguments based on the new valuations (cf. Figure 21), and compute the new coefficients. 


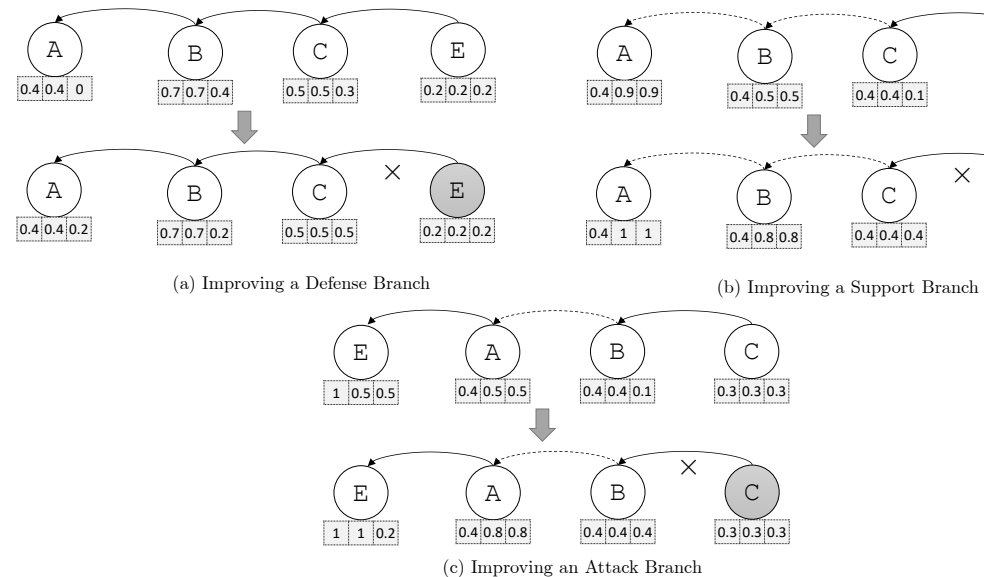

Figure 19: Improving Relations in $L-B A F$ through the pruning process.

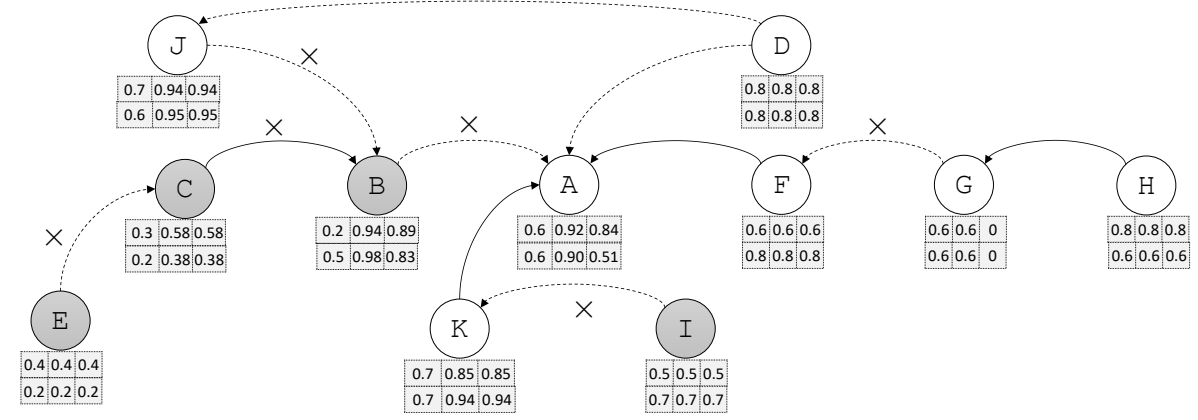

Figure 20: Pruning process over the labeled bipolar argumentation graph.

In this case, the particular conflict, support, and argument quality coefficients are: $\omega_{\mathrm{A}}^{\mathrm{a}}=0.22$, $\omega_{\mathrm{A}}^{\mathrm{s}}=0.28$, and $\omega_{\mathrm{A}}^{\mathrm{q}}=0.68$ (w.r.t. reliability) and $\omega_{\mathrm{B}}^{\mathrm{a}}=0.25, \omega_{\mathrm{B}}^{\mathrm{s}}=0.35$, and $\omega_{\mathrm{B}}^{\mathrm{q}}=0.67$ (w.r.t. relevance). The general conflict, support, and argument quality coefficients are: $\Omega^{\mathrm{a}}=0.24, \Omega^{\mathrm{s}}=$ 0.32 , and $\Omega^{\mathrm{q}}=0.68$.

Therefore, the refinement process depends on the user modeling decisions to both define the cutoff thresholds and decide when an optimal argumentation framework has been achieved. As we can see, the support and quality coefficient are closely related; this means that, if the argument qualities increase, then the support between them may become irrelevant at some point. Thus, to analyze the argumentative discussion, we need to put special attention to the attack coefficient to analyze the negative relation, and the support or quality coefficients, to analyze the positive relation. Next, we classify arguments $\mathrm{AR}^{\prime}$ according to Definition 13 as follows:

$$
\begin{aligned}
\mathrm{AR}_{n} & =\{\mathrm{G}\}(\text { neutralized }), \\
\mathrm{AR}_{w} & =\emptyset(\text { weakened }), \\
\mathrm{AR}_{s} & =\{\mathrm{A}, \mathrm{J}\}(\text { strengthened }), \text { and } \\
\mathrm{AR}_{u} & =\{\mathrm{D}, \mathrm{H}, \mathrm{K}\} \text { (unchallenged }) .
\end{aligned}
$$




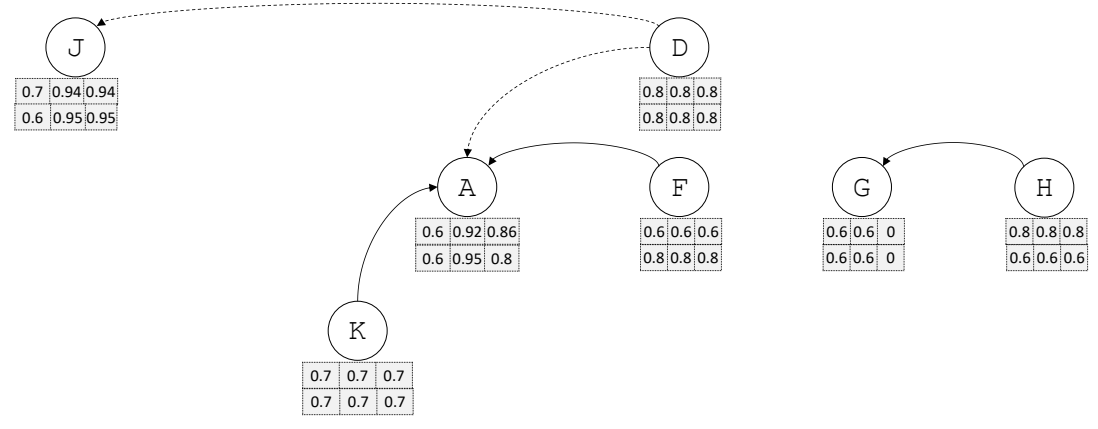

Figure 21: Example of a labeled bipolar argumentation graph.

Finally, we refine the analysis over each set by applying Definition 15 to obtain acceptability extensions specifying sets of acceptable arguments: $\mathrm{AR}_{s}=\{\mathrm{A}, \mathrm{J}\}$ is a CF-S, S-S, and C-S extension, since it is a conflict-free, safe, and closed under $\mathcal{R}_{s}$, while the set $\mathrm{AR}_{u}=\{\mathrm{D}, \mathrm{H}, \mathrm{K}\}$ is a CF-U extension and $S$ - $U$ extension, but it is not a $C$ - $U$ extension-the relation $\mathrm{AR}_{u}$ is not closed under $\mathcal{R}_{s}$ because $\mathrm{D}$ supports $\mathrm{A}$, but $\mathrm{A}$ does not belong to $\mathrm{AR}_{u}$. Also, $\mathrm{D}$ supports $\mathrm{A}$, which is attacked by $\mathrm{K}$; however, $\mathrm{K} \nsucc \mathrm{A}$ because the features of $\mathrm{A}$ are better than those of $\mathrm{K}$ (the accuracy and reliability of $\mathrm{A}$ are 0.86 and 0.52 , while for $\mathrm{K}$ are 0.7 and 0.7 , respectively).

\section{Appendix B. Abstract and Structured Frameworks: Conflict Cycle Analysis}

As mentioned in the introduction, in this work, we only consider BAFs that are well-founded, i.e., those frameworks that do not contain cycles of any kind. The reason behind this assumption is that dealing with frameworks that are not well-founded in a principled manner introduces a considerable impact on the complexity of the formalism. Nevertheless, since dealing with cycles is an essential topic in argumentation, we will now discuss some ideas that can be applied in treating this kind of situations in our formalism, which we will more fully address in our future work. In the following discussion, we will only consider conflict cycles; though this may seem too restrictive, it should be noted that support cycles are mostly generated by so-called fallacious specifications (Cohen et al., 2014b).

\section{B.1 Dampening Factor}

An immediate alternative is the resolution of conflicts through the application of a fixed-point function that allows achieving stable values in the argumentative system; well-known examples of this kind of solution occur in the literature (Leite \& Martins, 2011; Potyka, 2018; Amgoud \& Ben-Naim, 2018). The drawback of this approach is its computational cost, the restrictive ability to represent knowledge behavior in particular application domains, and the approximation errors that the process might introduce in the arguments' valuations. However, it is a feasible solution to leverage the information provided by abstract frameworks; that is, if we do not have more information about the arguments, then this solution is a reasonable way to deal with cyclic relations. 


\section{B.2 Argument Structure and Value Propagation: Weakening and Aggregation in the Presence of Conflict Cycles}

In previous work (2017) we analyzed a formalism called Labeled Argumentation Frameworks (LAFs), which presents a flexible structure to represent an argumentative discussion to answer a query. This formalization uses the knowledge representation capabilities provided by the Argument Interchange Format (Chesñevar et al., 2006) and leverages an algebra of argumentation labels ${ }^{9}$ that allows representing the arguments' features through labels, propagating them in the argumentation domain through a series of operations defined for this purpose. Thus, the effects produced by the interactions of support, conflict, and aggregation among arguments are reflected by the operations defined in an algebra of labels.

In $L A F$, an argumentative graph ( $c f$. Figure 22) is represented via the AIF ontology; there exist information nodes (I-nodes) and scheme application nodes (S-nodes), where I-nodes are used to represent propositional information contained in an argument (claim and premise) and S-nodes capture the application of schemes, such as inference rule schemes (RA-nodes) and conflict schemes (CA-nodes). S-nodes can be further classified; for example, inference schemes can be deductive or defeasible, and defeasible inference schemes can be subdivided into more specific argumentation schemes (e.g., expert opinion or witness testimony, among others). Thus, inference and conflict schemes in the ontology embody the general principles expressing how it is that $\mathrm{X}$ is inferable from $\mathrm{W}$ and $\mathrm{X}$ is in conflict with $\sim \mathrm{X}$ ( $c f$. Figure 22 (a)), respectively. The individual RA-nodes and CA-nodes that fulfill these schemes then capture the passage or the process of actually inferring $Y$ from $\mathrm{X}$ and $\mathrm{T}$, and conflicting $\mathrm{T}$ with $\sim \mathrm{T}(c f$. Figure $22(\mathrm{c}))$, respectively. There are two types of edges: scheme edges emanate from S-nodes and connected with I-nodes, and data edges emanating from I-nodes and ending in S-nodes. Notice that edges connecting I-nodes are forbidden, because I-nodes cannot be connected without an explanation that justifies that connection. There is always a scheme, justification, inference, or rationale behind a relation between two or more I-nodes that is captured through an S-node. Moreover, only I-nodes can have zero incoming edges, as all S-nodes relate two or more components (for RA-nodes, at least one antecedent is used to support at least one conclusion; for CA-nodes, at least one claim is in conflict with at least one other).

In $L A F$, an argumentative graph can contain cycles produced by one or more conflicts between two or more knowledge pieces. For example, in Figure 22, there exists an even conflict between $\mathrm{A}$ and $\mathrm{B}$ (one in claims $\mathrm{x}$ and $\sim \mathrm{x}$, the other in claims $\mathrm{y}$ and $\sim \mathrm{y}$ ). Therefore, the labeling procedure must determine the restrictions that the valuations must fulfill by adequately considering these inconsistencies. The propagation process in $L A F$ does not use fixed-point functions to resolve cycles - the features associated with each piece of knowledge are the only tools used towards this end. Thus, the strength of arguments is obtained by the aggregation of the strengths associated with each independent reasoning chain supporting the conclusion. Also, the aggregation of arguments reduces the complexity of conflict resolution, since there exists a single point of conflict in the argumentation graph for each pair of contradictory formulas. Therefore, it is possible to implement a weakening relationship between contradictory arguments, since the weakened strength does not depend on the order in which the conflicts are taken into account. That is, the conflicting formulas are weakened in both directions, where the strongest conclusion prevails in a weakened state, and

9. The algebra proposed in the present work is characterized in order to work at an abstract level considering different kinds of relations and interpretations; thus, even though at first glance LAF and L-BAF may seem similar, the underlying intuitions, properties, definitions, and effects produced by the operations are very different. 


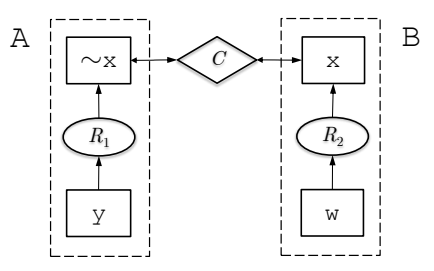

(a)

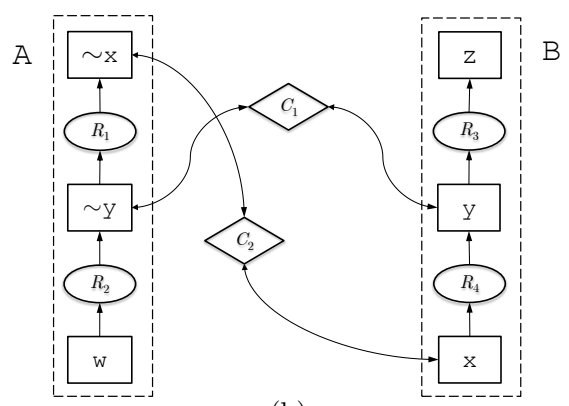

(b)
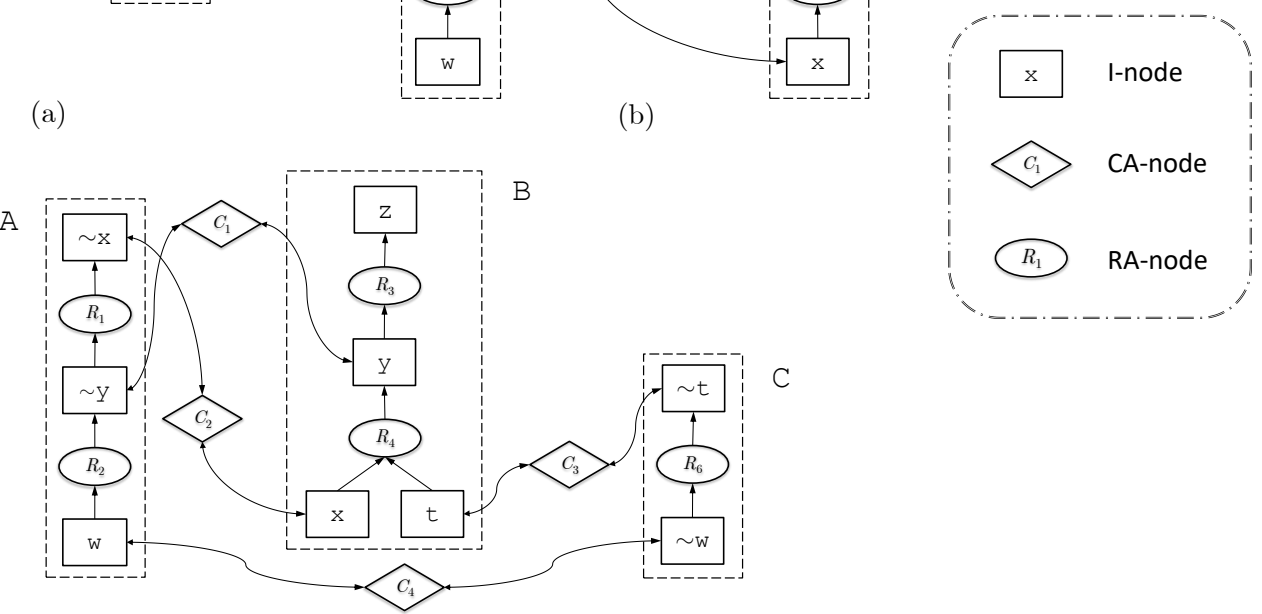

(c)

Figure 22: Cycles in a Labeled Argumentation Framework ( $L A F$, a semi-structured formalism).

the weakest conclusion is neutralized. In a $L A F$, we need a knowledge base to create the argumentation graph to analyze a specific domain; thus, the information provided is indeed more specific than the information provided in our abstract formalism. Note that two-way conflicts are taken into account; however, since the representation of knowledge is carried out on a directed graph, conflicts are directed.

\section{B.3 A New Proposal}

We would like to explore how valuations can spread through the abstract argumentation system in a more sophisticated and direct manner. As has been mentioned, we regard the fixed-point approaches as the natural result of working with abstract frameworks that have limited information available about the relations between arguments. The main question we wish to analyze in this section is then: Would it be possible to propagate the arguments' valuations more effectively if we had more information available about the relations between arguments, such as the conclusion that an argument supports? The structured framework presented in the previous section hints towards the fact that enriching the information in abstract argumentative frameworks a little further allows for richer ways of carrying out the labeling process in the presence of cycles. Towards this end, one possibility is to analyze how this less abstract, semi-structured formalism can solve the conflict situation presented at an abstract level by identifying the minimal elements that we need to perform a more sophisticated propagation.

First, we claim that it is necessary to have a greater granularity in relation to the argumentative entities, being able to differentiate the levels of subarguments and the logical conclusions that they 


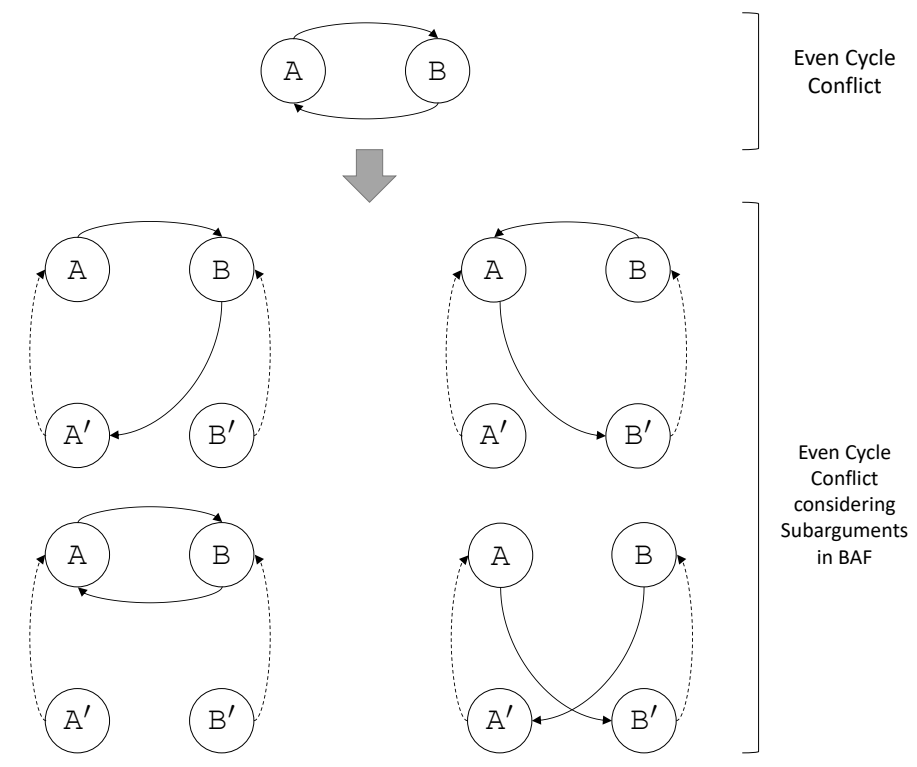

Figure 23: Even Cycles in a Meta-Bipolar Argumentation Framework.

support. In this direction, we consider the work presented by Gorogiannis and Hunter (2011), where an abstract argumentation framework is instantiated to a classical logical system satisfying a set of properties and postulates. In addition, Martinez and Rotstein (2008) have argued that an essential element in defeasible argumentation, which has not been widely explored as an extension in the abstract setting, is the consideration of sub-arguments, i.e., inner portions of an argument that are arguments on their own; nevertheless, sub-arguments constitute a vital part of their enclosing structure, usually called super-arguments. As sub-arguments are also arguments, they verify the same properties as any other argument in the system. To address the problem, these authors introduced an abstract sub-argument relation leading to an extended argumentation framework where this relation is considered. The formalization is centered on a set of properties and characterizations of a "sensible" sub-argumentation relation. Finally, the matter of argumentation semantics is addressed, analyzing the role of sub-arguments when computing the set of acceptable arguments, independently of the chosen semantics. In this direction, Prakken (2014) extends abstract argumentation frameworks considering a necessary support relation to model sub-argument relations. Thus, focusing on how cycles are treated in $L A F$, we will study how we could address bipolar argumentation cycles in our formalism without losing generality. In other words, we evaluate how the resolution of cycles, as done in $L A F$, can be carried over to the abstract level by leveraging specific information about the domain.

Considering the advantages presented in both of the mentioned research lines, we are working on building a meta-level bipolar argumentation framework in which two significant elements are considered: the notion of abstract sub-argument in the bipolar domain, and the conclusion that supports each argument. The goal is to create a meta-bipolar argumentation framework that is closed under the notion of sub-argument; this procedure will allow the identification of the essential pieces necessary for the framework. Indeed, we must consider the sub-argument notion as a special form of support (Prakken, 2014); thus, if an argument $\mathrm{A}^{\prime}$ is a sub-argument of $\mathrm{A}$, then $\mathrm{A}^{\prime}$ supports $\mathrm{A}$. 


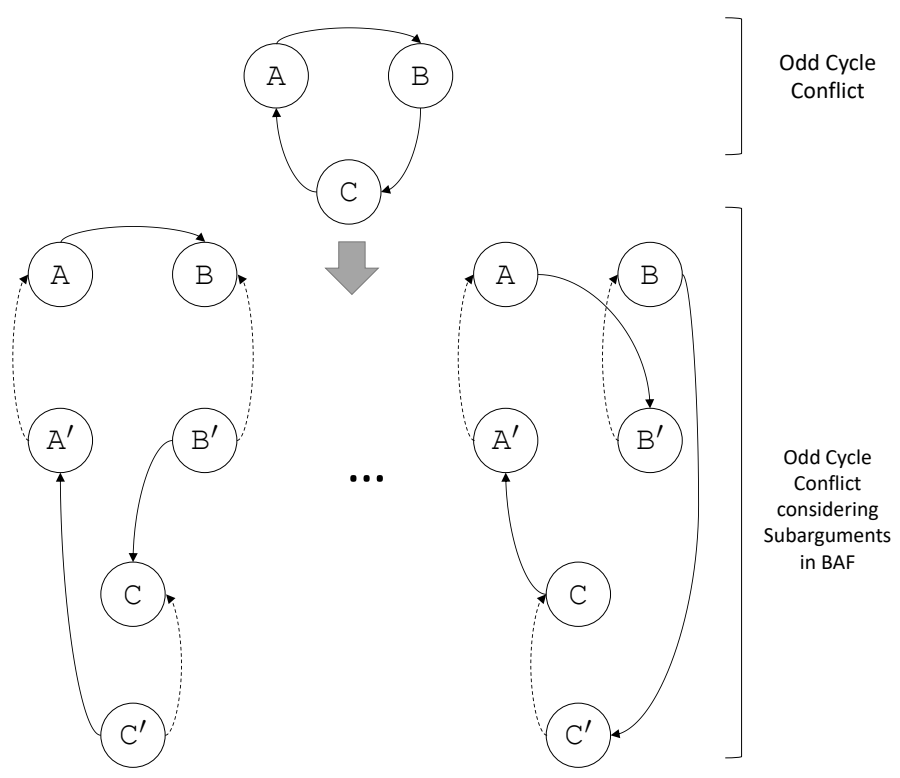

Figure 24: Odd Cycles in a Meta-Bipolar Argumentation Framework.

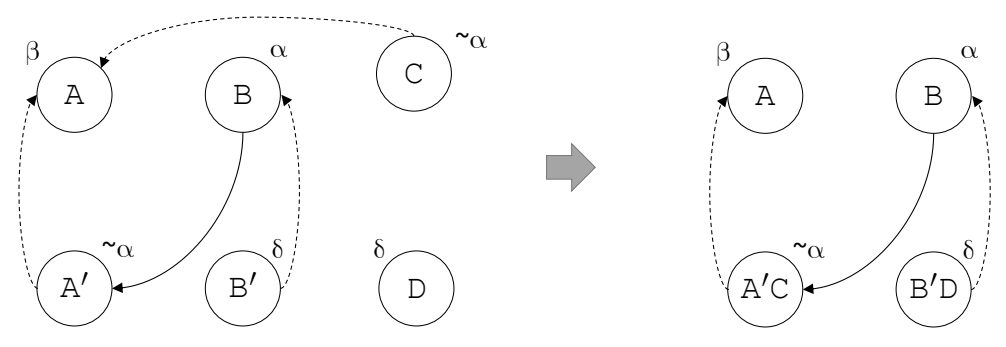

Figure 25: Aggregation of Arguments in a Meta-Bipolar Argumentation Framework.

However, a significant problem must be addressed: how are attacks disaggregated? The question arises because before introducing the notion of sub-argument, the attack between two arguments in the original bipolar argumentation framework can reflect different situations (see Figure 23 and Figure 24, where dashed lines represent the subargument relation, interpreted as support in BAF). Thus, to avoid this problem, we label each argument with the corresponding claim that represents the reason behind argument support. Then, considering this new information, we can carry out two important procedures: arguments supporting the same conclusions (we can deduce that, as in $L A F$, the aggregation of arguments may reduce the complexity of conflict resolution), and identify the conflict points between arguments, providing a specific way to avoid the disaggregation of attacks (see Figure 25).

Given the above preliminary analysis, we conjecture that implementing the algebra of argumentation labels presented in this work with some refinement over the meta-level bipolar framework is possible. In future work, we will study different approaches to bipolar argumentation and characterize the properties that the corresponding algebras satisfy in the extended domain. 


\section{Appendix C. Proofs}

Proposition 1 The worst-case running time of the labeling procedure for the bipolar argumentation graph $\mathrm{G}_{\Theta}$ is $O(n \times(m+t))$, where $n$ is the cardinality associated with the set of arguments $\mathrm{AR}, m$ is the maximal number of arguments with an attacking role for an argument $\mathrm{X} \in \mathrm{AR}$, and $t$ is the maximal number of arguments with a supporting role for an argument $\mathrm{X} \in \mathrm{AR}$.

Proof: Intuitively, as a loose upper bound, we can say that the labeling process for an argumentation graph $\mathrm{G}_{\Theta}$, associated with the underlying $B A F \Theta$, has a worst-case running time in $O\left(n^{3}\right)$. This arises from the following analysis: first, we need to label each argument (node in the graph $\mathrm{G}_{\Theta}$ ) $(O(n))$; second, for each argument we must analyze all the support argument associated with it $(O(n-1))$; third, for each argument we must analyze all the attacks associated with it $(O(n-1))$.

However, let $t$ the cardinality of $(\mathrm{X})$ representing the set of supporting arguments for an argument $\mathrm{X} \in \mathrm{AR}$, and $m$ the cardinality of $\overrightarrow{\mathrm{X})}$ representing the attacking arguments for an argument $\mathrm{X} \in \mathrm{AR}$. First, we can refine the above analysis arguing that the worst case for the labeling of each argument $\mathrm{X} \in \mathrm{AR}$ is $O(m+t)$. This analysis arises based on the fact that, to label an argument X is necessary label the arguments supporting and attacking X. Thus, the labeling process applied to X produces a labeling over the supporting and attacking arguments. Finally, we can conclude that the labeling process for an argumentation graph $\mathrm{G}_{\Theta}$ has a order $O(n \times(m+t))$.

Theorem 1 Let $\Psi=\langle\Theta, \mathbf{A}, v\rangle$ be an $L-B A F$ where $\Theta$ is the underlying BAF, $\mathrm{G}_{\Theta}$ be the corresponding argumentation graph for $\Theta$, and $\lambda_{1}(\Theta)$ and $\lambda_{2}(\Theta)$ be two different labeling sequences for $\mathrm{G}_{\Theta}$, where $\lambda_{1}(\Theta)$ generates system $E Q S_{1}$ and $\lambda_{2}(\Theta)$ generates system $E Q S_{2}$. Then, we have that $E Q S_{1}=E Q S_{2}$.

Proof: Let us suppose that $E Q S_{1} \neq E Q S_{2}$. Then, either $E Q S_{1} \nsubseteq E Q S_{2}$, or $E Q S_{2} \nsubseteq E Q S_{1}$, or both; assume, without loss of generality, that $E Q S_{1} \nsubseteq E Q S_{2}$. By Definition 11, the labeling procedure does not depend on the order in which arguments and attacks are considered, but this procedure depends on the relations (support and conflict) between the arguments of AR in $\Theta$. In this way, if there exists an equation in $E Q S_{1}$ that is not in $E Q S_{2}$, then there exists a relation analyzed by sequence $\lambda_{1}(\Theta)$ that was not analyzed by sequence $\lambda_{2}(\Theta)$ - this is a contradiction, since $\lambda_{1}(\Theta)$ and $\lambda_{2}(\Theta)$ are sequences obtained by a labeling process that explores the entire argumentative graph $\mathrm{G}_{\Theta}$ (in depth and breadth).

Lemma 1 Let $\Psi=\langle\Theta, \mathbf{A}, v\rangle$ be an $L-B A F$ where $\Theta$ is the underlying $B A F, \mathrm{G}_{\Psi}$ be the associated labeled bipolar graph, and $\mathrm{X}$ be an argument in $\mathrm{G} \Psi$. Then, for every algebra $\mathrm{A} \in \mathbf{A}$, the labels $\left\langle\alpha^{\mathrm{X}}\right.$, $\left.\mu^{\mathrm{X}}, \delta^{\mathrm{X}}\right\rangle$ related to $\mathrm{A}$, satisfy: (i) $\alpha^{\mathrm{X}} \in[\perp, \top]$, (ii) $\mu^{\mathrm{X}} \in\left[\alpha^{\mathrm{X}}, \top\right]$, and (iii) $\delta^{\mathrm{X}} \in\left[\perp, \mu^{\mathrm{X}}\right]$.

Proof: Considering Definition 8, a label $\alpha$ belong to a set of labels $\mathfrak{L}$ where there exists a partial order $\leq$ such that $T$ is the last label with respect to $\leq$ while $\perp$ is the first one. Hence, based on this notion, we analyze the effect associated with the positive (support and aggregation) and negative (conflict) relations. Then, we have that: 
(i) $\alpha^{\mathrm{A}} \in[\perp, \top]$. By Definition $11, \alpha^{\mathrm{A}}$ is given by the posture function $\rho: \mathrm{AR} \longrightarrow \mathrm{A}$, i.e., $\alpha^{\mathrm{A}}=$ $\rho(\mathrm{A})$. Furthermore, by Definition 9 and Definition 8, we have that the image associated with the function $\rho$ is the range $[\perp, \top]$. Thus, $\alpha^{\mathrm{A}}$ must belong to the interval $[\perp, \top]$.

(ii) $\mu^{\mathrm{A}} \in\left[\alpha^{\mathrm{A}}, \top\right]$. By Lemma $2-(i)$ we know that $\mu^{\mathrm{A}} \geq \alpha^{\mathrm{A}}$. Hence, we must prove that $\mu^{\mathrm{A}} \leq \top$. By Definition 11, the aggregated valuation $\mu^{\mathrm{A}}$ is obtained in the following two ways:

- If $(\overrightarrow{\mathrm{A}}) \neq \emptyset$, then $\mu^{\mathrm{A}}=\alpha^{\mathrm{A}} \oplus\left(\odot_{j=1}^{n} \delta^{\mathrm{A}_{j}}\right)$, with $\mathrm{A}_{j} \in(\overrightarrow{\mathrm{A}})$. By Definition 8 , if $\left(\odot_{j=1}^{n} \delta^{\mathrm{A}_{j}}\right)=\top$, then $\alpha^{\mathrm{A}} \oplus \top=\top$ since $\top$ is the absorbing element for $\oplus$ (Definition 8). Thus, $\mu^{\mathrm{A}}=\top$ which is the upper bound in the admitted range. One another hand, by Definition 8 , if $\left(\odot_{j=1}^{n} \delta^{\mathrm{A}_{j}}\right)=\perp$, then $\alpha^{\mathrm{A}} \oplus \perp=\alpha^{\mathrm{A}}$ since $\perp$ is the neutral element for $\oplus$ (Definition 8). Thus, $\mu^{\mathrm{A}}=\alpha^{\mathrm{A}}$ which is the lower bound in the admitted range.

- If $\overrightarrow{(\mathrm{A})}=\emptyset$, then $\mu^{\mathrm{A}}=\alpha^{\mathrm{A}}$. By $(i)$ we know that $\alpha^{\mathrm{A}} \leq \top$.Then, $(i i)$ holds.

(iii) $\delta^{\mathrm{A}} \in\left[\perp, \mu^{\mathrm{A}}\right]$. By Lemma $2-($ ii $)$ we know that $\mu^{\mathrm{A}} \geq \delta^{\mathrm{A}}$. Hence, we must prove that $\perp \leq \delta^{\mathrm{A}}$. By Definition 11, the overall valuation $\delta^{\mathrm{A}}$ is obtained in the following three ways:

- If $\overrightarrow{(\mathrm{A})} \neq \emptyset$ and $\overrightarrow{(\mathrm{A})}=\emptyset$, then $\delta^{\mathrm{A}}=\alpha^{\mathrm{A}} \ominus\left(\otimes_{k=1}^{n} \delta^{\mathrm{x}_{k}}\right)$ with $\mathrm{X}_{k} \in \overrightarrow{(\mathrm{A})}$. Thus, by definition 8 we know that if $\alpha^{\mathrm{A}} \leq \otimes_{k=1}^{n} \delta^{\mathrm{X}_{k}}$ then $\delta^{\mathrm{A}}=\perp$. Otherwise, $\delta^{\mathrm{A}}>\perp$. Then, (iii) holds.

- If $\overrightarrow{(A)} \neq \emptyset$ and $\overrightarrow{(A)} \neq \emptyset$, then we must analyze the following cases:

- If $\otimes_{k=1}^{n} \delta^{\mathrm{x}_{k}} \leq \odot_{j=1}^{m} \delta^{\mathrm{A}_{j}}$, then $\delta^{\mathrm{A}}=\alpha^{\mathrm{A}} \oplus\left(\odot_{j=1}^{m} \delta^{\mathrm{A}_{j}} \ominus \otimes_{k=1}^{n} \delta^{\mathrm{X}_{k}}\right)$ with $\mathrm{X}_{k} \in \overrightarrow{(\mathrm{A})}$ and $\mathrm{A}_{j} \in(\overrightarrow{\mathrm{A}})$. By $(i)$ we know that $\alpha^{\mathrm{A}} \geq \perp$. Then, $\delta^{\mathrm{A}} \geq \perp$. Thus, (iii) holds.

- If $\otimes_{k=1}^{n} \delta^{\mathrm{x}_{k}}>\odot_{j=1}^{m} \delta^{\mathrm{A}_{j}}$, then $\delta^{\mathrm{A}}=\alpha^{\mathrm{A}} \ominus\left(\otimes_{k=1}^{n} \delta^{\mathrm{x}_{k}} \ominus \odot_{j=1}^{m} \delta^{\mathrm{A}_{j}}\right)$ with $\mathrm{X}_{k} \in \overrightarrow{(\mathrm{A})}$ and $\mathrm{A}_{j} \in(\mathrm{A})$. If $\alpha^{\mathrm{A}} \leq\left(\otimes_{k=1}^{n} \delta^{\mathrm{x}_{k}} \ominus \odot_{j=1}^{m} \delta^{\mathrm{A}_{j}}\right)$ then $\delta^{\mathrm{A}}=\perp$. Otherwise, $\delta^{\mathrm{A}}>\perp$. Then, (iii) holds.

- If $\overrightarrow{(\mathrm{A})}=\emptyset$, then $\delta^{\mathrm{A}}=\mu^{\mathrm{A}} \geq \perp$. Thus, (iii) holds.

Lemma 2 Let $\Psi=\langle\Theta, \mathbf{A}, v\rangle$ be an $L-B A F$ where $\Theta$ is the underlying $B A F, \mathrm{G}_{\Psi}$ be the associated labeled bipolar graph, and $\mathrm{X}$ be an argument in $\mathrm{G}_{\Psi}$. Then, for every algebra $\mathrm{A} \in \mathrm{A}$, the labels $\left\langle\alpha^{\mathrm{X}}\right.$, $\left.\mu^{\mathrm{X}}, \delta^{\mathrm{X}}\right\rangle$ related to $\mathrm{A}$, satisfy:

(i) $\mu^{\mathrm{X}} \geq \alpha^{\mathrm{X}}$;

(ii) $\mu^{\mathrm{x}} \geq \delta^{\mathrm{x}}$; and

(iii) If $\mu^{\mathrm{x}}=\perp$, then $\delta^{\mathrm{x}}=\alpha^{\mathrm{x}}=\perp$.

Proof: Recall that an argument A has three associated valuations, $\alpha^{\mathrm{A}}, \mu^{\mathrm{A}}$ and $\delta^{\mathrm{A}}$, where $\alpha^{\mathrm{A}}$ is the original value of the attribute assigned to the argument by $v, \mu^{\mathrm{A}}$ accounts for the aggregation of the attributes of arguments supporting $\mathrm{A}$, and $\delta^{\mathrm{A}}$ is obtained after taking the attacks into account. Thus, the labeling procedure introduced in Definition 11 establishes a dependency between these valuations, since to be able to compute the overall valuation for the argument $\mathrm{A}$ it is necessary to 
know the accumulated valuations for A and the accumulated valuation associated with each attacking argument.

(i) $\mu^{\mathrm{A}} \geq \alpha^{\mathrm{A}}$. On one hand, if $(\overrightarrow{\mathrm{A}}) \neq \emptyset$, then by Definition 11 we know that $\mu^{\mathrm{A}}=\alpha^{\mathrm{A}} \oplus\left(\odot_{j=1}^{m} \delta^{\mathrm{A}_{j}}\right)$ with $\mathrm{A}_{j} \in \overrightarrow{(\mathrm{A})}$. Furthermore, by the conditions that a strengthening operator must satisfy introduced in Definition 8, we can deduce that $\alpha^{\mathrm{A}} \oplus\left(\odot_{j=1}^{m} \delta^{\mathrm{A}_{j}}\right) \geq \alpha^{\mathrm{A}}$ where $\mathrm{A}_{j}$ supports $\mathrm{A}$, and thus property $(i)$ holds. On another hand, if $\overrightarrow{(A)}=\emptyset$, then by Definition 11 we know that $\mu^{\mathrm{A}}=\alpha^{\mathrm{A}}$. Thus, $(i)$ holds.

(ii) $\mu^{\mathrm{A}} \geq \delta^{\mathrm{A}}$. We separate the proof in two parts:

- If $\overrightarrow{(\mathrm{A})} \neq \emptyset$, then by Definition 11 we know that $\mu^{\mathrm{A}}=\alpha^{\mathrm{A}} \oplus\left(\odot_{j=1}^{m} \delta^{\mathrm{A}_{j}}\right)$ with $\mathrm{A}_{j} \in(\overrightarrow{\mathrm{A}})$. Furthermore, if $\overrightarrow{(\mathrm{A})}=\emptyset$, then by Definition 11 we know that $\delta^{\mathrm{A}}=\mu^{\mathrm{A}}$. Thus, (ii) holds. Otherwise, if $\overrightarrow{(A)} \neq \emptyset$, then we must analyze the attack effect over the attacked argument as follows:

- If $\otimes_{k=1}^{n} \delta^{\mathrm{x}_{k}} \leq \odot_{j=1}^{m} \delta^{\mathrm{A}_{j}}$, with $\mathrm{X}_{k} \in \overrightarrow{(\mathrm{A})}$ and $\mathrm{A}_{j} \in(\overrightarrow{\mathrm{A}})$, then by Definition 11 we know that $\delta^{\mathrm{A}}=\alpha^{\mathrm{A}} \oplus\left(\odot_{j=1}^{m} \delta^{\mathrm{A}_{j}} \ominus \otimes_{k=1}^{n} \delta^{\mathrm{X}_{k}}\right)$. Since $\odot{ }_{j=1}^{m} \delta^{\mathrm{A}_{j}} \geq\left(\odot_{j=1}^{m} \delta^{\mathrm{A}_{j}} \ominus \otimes_{k=1}^{n} \delta^{\mathrm{X}_{k}}\right)$, we can deduce that $\mu^{\mathrm{A}} \geq \delta^{\mathrm{A}}$. Thus, (ii) holds.

- If $\otimes_{k=1}^{n} \delta^{\mathrm{x}_{k}}>\odot_{j=1}^{m} \delta^{\mathrm{A}_{j}}$, with $\mathrm{X}_{k} \in \overrightarrow{(\mathrm{A})}$ and $\mathrm{A}_{j} \in \overrightarrow{(\mathrm{A})}$, then by Definition 11 we know that $\delta^{\mathrm{A}}=\alpha^{\mathrm{A}} \ominus\left(\otimes_{k=1}^{n} \delta^{\mathrm{X}_{k}} \ominus \odot_{j=1}^{m} \delta^{\mathrm{A}_{j}}\right)$. Since $\otimes_{k=1}^{n} \delta^{\mathrm{X}_{k}}>\odot_{j=1}^{m} \delta^{\mathrm{A}_{j}}$, and by the conditions that a conflict operator must satisfy introduced in Definition 8 , we have that $\delta^{\mathrm{A}}<\alpha^{\mathrm{A}} \leq \mu^{\mathrm{A}}$. Thus, we can deduce that $\delta^{\mathrm{A}}<\mu^{\mathrm{A}}$. Thus, (ii) holds.

- If $\overrightarrow{(\mathrm{A})}=\emptyset$, then by Definition 11 we know that $\mu^{\mathrm{A}}=\alpha^{\mathrm{A}}$. If $\left.\overrightarrow{\mathrm{A}}\right) \neq \emptyset$, then by Definition 11 we know that $\delta^{\mathrm{A}}=\alpha^{\mathrm{A}} \ominus\left(\otimes_{k=1}^{n} \delta^{\mathrm{X}_{k}}\right)<\alpha^{\mathrm{A}}=\mu^{\mathrm{A}}$. Thus, we have that $\delta^{\mathrm{A}}<\mu^{\mathrm{A}}$, and $(i i)$ holds. Otherwise if $\overrightarrow{(\mathrm{A})}=\emptyset$, then by Definition 11 we know that $\delta^{\mathrm{A}}=\mu^{\mathrm{A}}$. Thus, (ii) holds.

(iii) If $\mu^{\mathrm{A}}=\perp$, then $\delta^{\mathrm{A}}=\alpha^{\mathrm{A}}=\perp$. We separate the proof in the following points:

- If $\overrightarrow{(\mathrm{A})}=\emptyset$ and $\overrightarrow{(\mathrm{A})}=\emptyset$, then by Definition 11 we know that $\mu^{\mathrm{A}}=\alpha^{\mathrm{A}}$ and $\delta^{\mathrm{A}}=\mu^{\mathrm{A}}$. By hypothesis we know that $\mu^{\mathrm{A}}=\perp$. Thus, (iii) holds.

- If $\overrightarrow{(\mathrm{A})} \neq \emptyset$, then by Definition 11 we know that $\mu^{\mathrm{A}}=\alpha^{\mathrm{A}} \oplus\left(\odot_{j=1}^{m} \delta^{\mathrm{A}_{j}}\right)$ with $\mathrm{A}_{j} \in \overrightarrow{(\mathrm{A})}$. But, by hypothesis, $\mu^{\mathrm{A}}=\perp$. Then, $\mu^{\mathrm{A}}=\alpha^{\mathrm{A}} \oplus\left(\odot_{j=1}^{m} \delta^{\mathrm{A}_{j}}\right)=\perp$.

Thus, we can deduce that $\alpha^{\mathrm{A}}=\perp$ and $\odot_{j=1}^{m} \delta^{\mathrm{A}_{j}}=\perp$ where $\perp$ is the neutral element for the operator $\oplus$ as it is established in Definition 8. Furthermore, we must analyze the following situation:

- If $\overrightarrow{(\mathrm{A})}=\emptyset$, then by Definition 11 we know that $\delta^{\mathrm{A}}=\mu^{\mathrm{A}}=\perp$. Thus, (iii) holds.

- If $\overrightarrow{(\mathrm{A})} \neq \emptyset$, then by Definition 11 we know that: $(a)$ If $\otimes_{k=1}^{n} \delta^{\mathrm{X}_{k}} \leq \odot_{j=1}^{m} \delta^{\mathrm{A}_{j}}$ with $\mathrm{X}_{k} \in \overrightarrow{(\mathrm{A})}$ and $\mathrm{A}_{j} \in(\overrightarrow{\mathrm{A}})$, then $\delta^{\mathrm{A}}=\alpha^{\mathrm{A}} \oplus\left(\odot_{j=1}^{m} \delta^{\mathrm{A}_{j}} \ominus \otimes_{k=1}^{n} \delta^{\mathrm{x}_{k}}\right)$. But, since $\odot_{j=1}^{m} \delta^{\mathrm{A}_{j}}=$ 
$\perp$ we can deduce that $\otimes_{k=1}^{n} \delta^{\mathrm{X}_{k}}=\perp$. Then, $\delta^{\mathrm{A}}=\perp$. Thus, (iii) holds; (b) If $\otimes_{k=1}^{n} \delta^{\mathrm{X}_{k}}>\odot_{j=1}^{m} \delta^{\mathrm{A}_{j}}$ with $\mathrm{X}_{k} \in \overrightarrow{(\mathrm{A})}$ and $\mathrm{A}_{j} \in(\overrightarrow{\mathrm{A}})$, then $\delta^{\mathrm{A}}=\alpha^{\mathrm{A}} \ominus\left(\otimes_{k=1}^{n} \delta^{\mathrm{X}_{k}} \ominus \odot_{j=1}^{m} \delta^{\mathrm{A}_{j}}\right)$. But, we already proved that $\alpha^{\mathrm{A}}=\perp$, and then we obtain that $\delta^{\mathrm{A}}=\perp$. Thus, (iii) holds.

- If $\overrightarrow{(\mathrm{A})}=\emptyset$ and $\overrightarrow{(\mathrm{A})} \neq \emptyset$, then $\mu^{\mathrm{A}}=\alpha^{\mathrm{A}}$. Furthermore, by hypothesis we know that $\mu^{\mathrm{A}}=\perp$. Thus, we can deduce that $\alpha^{\mathrm{A}}=\perp$. On another hand, by Definition 11 we know that $\delta^{\mathrm{A}}=\alpha^{\mathrm{A}} \ominus\left(\otimes_{k=1}^{n} \delta^{\mathrm{x}_{k}}\right)$ with $\mathrm{X}_{k} \in \overrightarrow{(\mathrm{A})}$. But, $\alpha^{\mathrm{A}}=\perp$ then $\delta^{\mathrm{A}}=\perp$. Thus, (iii) holds.

Theorem 2 Let $\Psi=\langle\Theta, \mathbf{A}, v\rangle$ be an $L-B A F$ where $\Theta$ is the underlying BAF, $\mathrm{G}_{\Theta}$ be the corresponding argumentation graph for $\Theta$, and $\Lambda_{\Psi}$ be the set of all the valid labelings for $\Psi$. Then, $\Lambda_{\Psi}$ is sound and complete.

Proof: We need to show that: all the labelings that belong to $\Lambda_{\Psi}$ are adequate solutions for $G_{\Theta}$ (soundness), and if there exists a valid labeling for a bipolar argumentation graph $\mathrm{G}_{\Theta}$, that labeling belongs to $\Lambda_{\Psi}$ (completeness).

$\Lambda_{\Psi}$ (Soundness). By hypothesis $\mathrm{G}_{\Theta}$ is the corresponding argumentation graph for $\Theta$ and $\lambda(\Theta)$ is a labeling for $\mathrm{G}_{\Theta}$.

Furthermore, by Definition 11, we obtain the system of equations EQS representing the constraints of all the valuations associated with the arguments of $\mathrm{G}_{\Theta}$. That is, $E Q S$ represents the arguments behavior in the argumentative graph $\mathrm{G}_{\Theta}$. In addition, by Theorem 1 the system $E Q S$ associated with a graph $\mathrm{G}_{\Theta}$ is unique. Hence, if $\lambda(\Theta)$ is a set of values $\alpha^{\mathrm{A}}, \mu^{\mathrm{A}}$ and $\delta^{\mathrm{A}}$ that constitute a solution to $E Q S$, then by Definition 12 it is a valid labeling $\mathrm{G}_{\Theta}$. Thus, by construction $\lambda(\Theta)$ must be in $\Lambda_{\Psi}$.

$\Lambda_{\Psi}$ (Completeness). By hypothesis the labeling associated with $\mathrm{G}_{\Theta}$ is valid. Then, by Lemma 2 the original value associated with an argument is less or equal than the strengthened value, while the strengthened value is greater or equal than the weakened value. That is, arguments with supports are strengthened, while arguments with attackers are weakened. Thus, the arguments relations are reflected in their labels. Furthermore, by Lemma 1, the valuations associated with the arguments are always in the desired range.

Lemma 3 Let $\Psi=\langle\Theta, \mathbf{A}, v\rangle$ be an $L-B A F$, and $G_{\Psi}$ be the corresponding labeled bipolar argumentation graph. The gradual status assignment $\chi_{g}$ to $G_{\Psi}$ is unique.

Proof: Suppose that there exist two assignments $\chi_{g}$ and $\chi_{g}^{\prime}$ for a valid labeling associated with the bipolar graph $\mathrm{G}_{\Theta}$. Hence, there exists an argument $\mathrm{A} \in \mathrm{AR}$ with $\left\langle\alpha^{\mathrm{A}}, \mu^{\mathrm{A}}, \delta^{\mathrm{A}}\right\rangle$ the associated valuation such that $\mathrm{A}$ is associated with two statuses. Thus, it is possible to assign the following combination of status to $\mathrm{A}$ :

- Neutralized and Weakened. Contradiction, if A has assigned the status neutralized then $\delta^{\mathrm{A}}=$ $\perp$, while if A has assigned the status weakened then $\delta^{\mathrm{A}}>\perp$.

- Neutralized and Strengthened. Contradiction, if A has assigned the status neutralized then $\delta^{\mathrm{A}}=\perp$, while if A has assigned the status strengthened then $\delta^{\mathrm{A}}>\alpha^{\mathrm{A}}$. 
- Neutralized and Unchallenged. Contradiction, if A has assigned the status neutralized then $\delta^{\mathrm{A}}=\perp$, while if A has assigned the status unchallenged then $\alpha^{\mathrm{A}}=\delta^{\mathrm{A}} \neq \perp$.

- Weakened and Strengthened. Contradiction, if A has assigned the status weakened then $\alpha^{\mathrm{A}}>$ $\delta^{\mathrm{A}}>\perp$, while if A has assigned the status strengthened then $\delta^{\mathrm{A}}>\alpha^{\mathrm{A}}$.

- Weakened and Unchallenged. Contradiction, if A has assigned the status weakened then $\alpha^{\mathrm{A}}>$ $\delta^{\mathrm{A}}>\perp$, while if A has assigned the status unchallenged then $\delta^{\mathrm{A}}=\alpha^{\mathrm{A}}$.

- Strengthened and Unchallenged. Contradiction, if A has assigned the status strengthened then $\alpha^{\mathrm{A}}<\delta^{\mathrm{A}}$, while if A has assigned the status unchallenged then $\delta^{\mathrm{A}}=\alpha^{\mathrm{A}}$.

This leads us to a contradiction, any argument $A \in A R$ has a unique status based on a specific valid labeling associated with $\mathrm{G}_{\Theta}$.

Proposition 2 Let $\Psi=\langle\Theta, \mathbf{A}, v\rangle$ be an $L-B A F, G_{\Psi}$ be the corresponding labeled bipolar argumentation graph, $\chi_{g}$ be the gradual status assignment to the bipolar graph $G_{\Psi}$, and $\mathrm{AR}_{n}, \mathrm{AR}_{w}, \mathrm{AR}_{s}$, and $\mathrm{AR}_{u}$ be a disjoint set of neutralized, weakened, strengthened, and unchallenged arguments in $\Psi$. Then, the collection of subsets $\left\{\mathrm{AR}_{n}, \mathrm{AR}_{w}, \mathrm{AR}_{s}, \mathrm{AR}_{u}\right\}$ is a finite cover of $\mathrm{AR}$.

Proof: From hypothesis we have that the sets of neutralized, weakened, strengthened, and unchallenged arguments are subsets of AR. Thus, we must prove that the union of the collection of subsets $\left\{\mathrm{AR}_{n}, \mathrm{AR}_{w}, \mathrm{AR}_{s}, \mathrm{AR}_{u}\right\}$ is equal to $\mathrm{AR}$. Suppose that $\mathrm{AR}_{n} \cup \mathrm{AR}_{w} \cup \mathrm{AR}_{s} \cup \mathrm{AR}_{u} \neq \mathrm{AR}$. Hence, there exists an argument $A$ such that $A \in A R$ and $A \notin A R_{n} \cup \mathrm{AR}_{w} \cup \mathrm{AR}_{s} \cup \mathrm{AR}_{u}$. However, by Definition 13, any argument $A \in A R$ has assigned one of four possible statuses for each algebra, creating a vector with the acceptability of that argument with respect to each of the attributes, and receives as its acceptability degree the least degree of those that appear in the vector. Furthermore, by Lemma 3, we know that any argument $A \in A R$ has a unique status based on a specific valid labeling associated with $\mathrm{G}_{\Theta}$. This leads us to a contradiction, since if $A \in A R$ then it has assigned a unique status based on a specific valid labeling. Then, $A$ must belong to the collection of subsets $\left\{\mathrm{AR}_{n}, \mathrm{AR}_{w}, \mathrm{AR}_{s}, \mathrm{AR}_{u}\right\}$. Thus, we can conclude that $\mathrm{AR}_{n} \cup \mathrm{AR}_{w} \cup \mathrm{AR}_{s} \cup \mathrm{AR}_{u}=\mathrm{AR}$.

Proposition 3 Let $\Psi=\langle\Theta, \mathrm{A}, v\rangle$ be an $L-B A F, \succ$ be a preference relation over $\mathrm{AR}$, and $S \subseteq \mathrm{AR}$ be a set of arguments. Then:

(i) If $S$ is safe, then $S$ is conflict-free.

(ii) If $S$ is conflict-free and closed under support, then $S$ is safe.

Proof: We separate the proof in the two parts shown in the statement:

(i) If $S$ is safe, then $S$ is conflict-free. Suppose that $S$ is not conflict-free. Then, $\exists \mathrm{B}, \mathrm{C} \in S$ such that $(\mathrm{B}, \mathrm{C}) \in \mathcal{R}_{a}$ with $\mathrm{B} \succ \mathrm{C}$. By hypothesis, $S$ is safe. Thus, by Definition $14, \nexists \mathrm{A} \in \mathrm{AR}$ and $\nexists \mathrm{B}, \mathrm{C} \in S$ such that $(\mathrm{B}, \mathrm{A}) \in \mathcal{R}_{a}$ with $\mathrm{B} \succ \mathrm{A}$, and either there is a sequence of supports from $\mathrm{C}$ to $\mathrm{A}$, or $\mathrm{A} \in S$. This leads us to a contradiction, any argument that belongs to $S$ or is supported by $S$ cannot be effectively attacked by $S$ by safe definition. 
(ii) If $S$ is conflict free and closed for $\mathcal{R}_{s}$, then $S$ is safe. Suppose that $S$ is not safe. Thus, $\exists \mathrm{A} \in \mathrm{AR}$ and $\exists \mathrm{B}, \mathrm{C} \in S$ such that $(\mathrm{B}, \mathrm{A}) \in \mathcal{R}_{a}$ with $\mathrm{B} \succ \mathrm{A}$, and either there is a sequence of supports from $\mathrm{C}$ to $\mathrm{A}$, or A $\in S$. By hypothesis, $S$ is conflict free. Thus, by Definition $14, \nexists \mathrm{B}, \mathrm{C} \in S$ such that $(\mathrm{B}, \mathrm{C}) \in \mathcal{R}_{a}$ with $\mathrm{B} \succ \mathrm{C}$. Furthermore, the closure property establishes that $\forall \mathrm{A} \in S, \forall \mathrm{B} \in \mathrm{AR}$ if A $\mathcal{R}_{s}$ B then B $\in S$. This leads us to a contradiction, since any argument supported by $S$ belongs to $S$ and it is conflict free.

Proposition 4 Let $\Psi=\langle\Theta, A, v\rangle$ be an $L-B A F$, and $\mathrm{AR}_{w}, \mathrm{AR}_{s}$, and $\mathrm{AR}_{u}$ be the sets of weakened, strengthened, and unchallenged arguments associated with $\Psi$. Then:

(i) Any $S-W$ (resp. S-S, S-U) extension is also a CF-W (resp. CF-S, CF-U) extension;

(ii) Any CF-W (resp. CF-S, CF-U) extension closed under $\mathcal{R}_{s}$ is also a $S-W$ (resp. S-S, S-U) extension; and

(iii) Any $S$-W (resp. S-S, $S$ - $U$ ) extension closed under $\mathcal{R}_{\text {s }}$ is also a $C$-W (resp. $C$-S, $C$ - $U$ ) extension.

Proof: We separate the proof in three parts:

(i) Any $S-W$ (resp. $S-S, S-U$ ) extension $S$ is also a $C F-W$ (resp. CF-S, CF-U) extension. By Definition 15, we can say that $S$ is a maximal safe set. Furthermore, by Proposition 3-(i), we know that if $S$ is safe, then $S$ is conflict-free. Thus, we only need to prove that $S$ is a maximal conflict-free set. Suppose that there exists an argument $\mathrm{A} \notin S$, where $\nexists \mathrm{B} \in \mathrm{AR}$ such that $(\mathrm{B}, \mathrm{A}) \notin \mathcal{R}_{a}$ or $\mathrm{B} \nsucc \mathrm{A}$. This leads us to a contradiction, since any argument supported and defended by $S$ belongs to $S$.

(ii) Any $C F-W$ (resp. CF-S, CF-U) extension $S$ closed under $\mathcal{R}_{s}$ is also an $S-W($ resp. $S-S, S-U)$ extension. By Definition 15, we can say that $S$ is a maximal conflict free set closed under $\mathcal{R}_{s}$. Furthermore, by Proposition 3, we know that if $S$ is conflict free and closed for $\mathcal{R}_{s}$, then $S$ is safe. Thus, we only need to prove that $S$ is a maximal safe set. Suppose that there exists an argument $\mathrm{A} \notin S$, where $\nexists \mathrm{B}, \mathrm{C} \in S$ such that $(\mathrm{B}, \mathrm{A}) \in \mathcal{R}_{a}$ with $\mathrm{B} \succ \mathrm{A}$ and there is a sequence of support from $\mathrm{C}$ to $\mathrm{A}$ (thus $\mathrm{A} \notin S$ ). This leads us to a contradiction, since any argument defended by $S$ belongs to $S$.

(iii) Any $S$-W (resp. $S$-S, $S$ - $U$ ) extension $S$ closed under $\mathcal{R}_{s}$ is also a $C$-W (resp. $C$-S, $C$ - $U$ ) extension. By Definition 15, we can say that $S$ is a maximal safe set closed under $\mathcal{R}_{s}$. Furthermore, by Definition 15, a C-W (resp. C-S, C-U) extension is a maximal conflict-free set closed under $\mathcal{R}_{s}$. By $(i)$, we know that any S-W (resp. S-S, S-U) extension $S$ is also a CF-W (resp. CF-S, CF-U) extension. Thus, $S$ is a maximal conflict-free set. In addition, by hypothesis we know that $S$ is closed under $\mathcal{R}_{s}$. This leads us to conclude that $S$ is a C-W (resp. C-S, C-U) extension.

Proposition 5 Let $\Psi=\langle\Theta, \mathbf{A}, v\rangle$ be an $L-B A F, G_{\Psi}$ be the corresponding labeled bipolar argumentation graph, and $\mathrm{AR}_{s}, \mathrm{AR}_{u}$, and $\mathrm{AR}_{n}$ be the sets strengthened, unchallenged, and neutralized arguments associated with $\Psi$, respectively. If all arguments $\mathrm{A} \in G_{\Psi}$ are such that $\alpha^{\mathrm{A}}=\top$ when $\overrightarrow{(\mathrm{A})}=\emptyset$, and $\alpha^{\mathrm{A}}=\perp$ when $(\overrightarrow{\mathrm{A}}) \neq \emptyset$, then 
(i) $\mathrm{AR}_{s} \cup \mathrm{AR}_{u} \cup \mathrm{AR}_{n}=\mathrm{AR}$.

(ii) $\mathrm{AR}_{s}$ and $\mathrm{AR}_{u}$ are conflict-free;

Proof: We separate the proof in two parts:

(i) $\mathrm{AR}_{s} \cup \mathrm{AR}_{u} \cup \mathrm{AR}_{n}=\mathrm{AR}$. We analyze the following cases:

- Suppose that the argument $\mathrm{A} \in G_{\Psi}$ verifies that $\alpha^{\mathrm{A}}=\top$ when $(\mathrm{A})=\emptyset$. Thus, by Definition 11 we have that $\mu^{\mathrm{A}}=\alpha^{\mathrm{A}}=\top$.

If $\overrightarrow{(\mathrm{A})}=\emptyset$, then by Definition $11 \delta^{\mathrm{A}}=\mu^{\mathrm{A}}$. Thus, we have that $\delta^{\mathrm{A}}=\top$. Hence, by Definition 13, we have that $\mathrm{A} \in \mathrm{AR}_{u}$.

If $\overrightarrow{(\mathrm{A})} \neq \emptyset$ and $\overrightarrow{(\mathrm{A})}=\emptyset$, then by Definition 11 we know that $\delta^{\mathrm{A}}=\alpha^{\mathrm{A}} \ominus\left(\otimes_{k=1}^{n} \delta^{\mathrm{x}_{k}}\right)$ with $\mathrm{X}_{k} \in \overrightarrow{(\mathrm{A})}$. Thus, we must analyze two possibilities:

- If there exists an argument $\mathrm{A}_{j} \in \overrightarrow{(\mathrm{A})}$ such that verifies that $\delta^{\mathrm{A}_{j}}=\top$, then by Definition 11 and Definition 8 we know that $\delta^{\mathrm{A}}=\perp$. Hence, by Definition 13, we have that $\mathrm{A} \in \mathrm{AR}_{n}$.

- If any argument $\mathrm{X}_{k} \in \overrightarrow{(\mathrm{A})}$ verifies that $\delta^{\mathrm{X}_{\mathrm{k}}}=\perp$, then by Definition 11 and Definition 8 we have that $\delta^{\mathrm{A}}=\alpha^{\mathrm{A}}=\top$. Hence, by Definition 13 , we have that $\mathrm{A} \in \mathrm{AR}_{u}$.

- Suppose now that the argument $\mathrm{A} \in G \Psi$ verifies that $\alpha^{\mathrm{A}}=\perp$ when $\overrightarrow{(\mathrm{A})} \neq \emptyset$. Thus, we must analyze two possibilities:

If $\overrightarrow{(\mathrm{A})}=\emptyset$, then by Definition 11 we know that $\delta^{\mathrm{A}}=\mu^{\mathrm{A}}$, and $\mu^{\mathrm{A}}=\alpha^{\mathrm{A}} \oplus\left(\odot_{j=1}^{m} \delta^{\mathrm{A}_{j}}\right)$, with $\mathrm{A}_{j} \in(\overrightarrow{\mathrm{A}})$. Thus, $\delta^{\mathrm{A}}=\odot_{j=1}^{m} \delta^{\mathrm{A}_{j}}$.

- If any argument $\mathrm{B} \in(\overrightarrow{\mathrm{A}})$ such that verifies that $\delta^{\mathrm{B}}=\top$, then by Definition 8 we know that $\delta^{\mathrm{A}}=\top$. Hence, by Definition 13, we have that $\mathrm{A} \in \mathrm{AR}_{s}$.

- If there exists an argument $\mathrm{B} \in \overrightarrow{(\mathrm{A})}$ such that verifies that $\delta^{\mathrm{B}}=\perp$, then by Definition 8 we know that $\delta^{\mathrm{A}}=\perp$. Hence, by Definition 13, we have that $\mathrm{A} \in \mathrm{AR}_{n}$.

If $\overrightarrow{(A)} \neq \emptyset$, then by by Definition 11 :

- If $\otimes_{k=1}^{n} \delta^{\mathrm{X}_{k}}=\odot_{j=1}^{m} \delta^{\mathrm{A}_{j}}$ with $\mathrm{X}_{k} \in \overrightarrow{(\mathrm{A})}$ and $\mathrm{A}_{j} \in \overrightarrow{(\mathrm{A})}$, then $\delta^{\mathrm{A}}=\alpha^{\mathrm{A}} \oplus\left(\odot_{j=1}^{m} \delta^{\mathrm{A}_{j}} \ominus\right.$ $\left.\otimes_{k=1}^{n} \delta^{\mathrm{X}_{k}}\right)$. Thus, $\delta^{\mathrm{A}}=\perp$. Hence, by Definition 13, we can deduce that $\mathrm{A} \in \mathrm{AR}_{n}$.

- If $\otimes_{k=1}^{n} \delta^{\mathrm{X}_{k}}<\odot_{j=1}^{m} \delta^{\mathrm{A}_{j}}$ with $\mathrm{X}_{k} \in \overrightarrow{(\mathrm{A})}$ and $\mathrm{A}_{j} \in(\overrightarrow{\mathrm{A}})$, then $\delta^{\mathrm{A}}=\alpha^{\mathrm{A}} \oplus\left(\odot_{j=1}^{m} \delta^{\mathrm{A}_{j}} \ominus\right.$ $\left.\otimes_{k=1}^{n} \delta^{\mathrm{X}_{k}}\right)$. Thus, $\delta^{\mathrm{A}}=\odot_{j=1}^{m} \delta^{\mathrm{A}_{j}} \ominus \otimes_{k=1}^{n} \delta^{\mathrm{X}_{k}}$ with $\mathrm{X}_{k} \in \overrightarrow{(\mathrm{A})}$ and $\mathrm{A}_{j} \in(\overrightarrow{\mathrm{A}})$. We need to have that any argument $\mathrm{A}_{j} \in \overrightarrow{(\mathrm{A})}$ satisfies that $\delta^{\mathrm{A}_{j}}=\top$. It is a consequence of if there exists an argument $\mathrm{A}_{l} \in(\overrightarrow{\mathrm{A}})$ that verifies that $\delta^{\mathrm{A}_{l}}=\perp$ with $1 \leq l \leq m$, then $\odot_{j=1}^{m} \delta^{\mathrm{A}_{j}}=\perp$. Contradiction. Furthermore, any argument $\mathrm{X}_{\mathrm{k}} \in \overrightarrow{(\mathrm{A})}$ must verify that $\delta^{\mathrm{x}_{k}}=\perp$, thus $\otimes_{k=1}^{n} \delta^{\mathrm{x}_{k}}=\perp$. Finally, by Definition 8 we have that $\delta^{\mathrm{A}}=\top$. Hence, by Definition 13, we can deduce that $\mathrm{A} \in \mathrm{AR}_{s}$. 
- If $\otimes_{k=1}^{n} \delta^{\mathrm{x}_{k}}>\odot_{j=1}^{m} \delta^{\mathrm{A}_{j}}$ with $\mathrm{X}_{k} \in \overrightarrow{(\mathrm{A})}$ and $\mathrm{A}_{j} \in \overrightarrow{(\mathrm{A})}$, then $\delta^{\mathrm{A}}=\alpha^{\mathrm{A}} \ominus\left(\otimes_{k=1}^{n} \delta^{\mathrm{x}_{k}} \ominus\right.$ $\left.\odot_{j=1}^{m} \delta^{\mathrm{A}_{j}}\right)$. Thus, by Definition 8 we have that $\delta^{\mathrm{A}}=\perp$. Hence, by Definition 13, we can deduce that $\mathrm{A} \in \mathrm{AR}_{n}$.

Therefore given an argument $\mathrm{A} \in G_{\Psi}$, we proved that $\mathrm{A} \in \mathrm{AR}_{S} \cup \mathrm{AR}_{u} \cup \mathrm{AR}_{n}$. Thus, we can conclude that $\mathrm{AR}_{s} \cup \mathrm{AR}_{u} \cup \mathrm{AR}_{n}=\mathrm{AR}$.

(ii) $\mathrm{AR}_{s} \cup \mathrm{AR}_{u}$ is conflict-free. By $(i)$, we know that $\mathrm{AR}_{s} \cup \mathrm{AR}_{u} \cup \mathrm{AR}_{n}=\mathrm{AR}$. Thus, we can affirm that $\mathrm{AR}_{w}=\emptyset$ since there does not exist an argument $\mathrm{A} \in \mathrm{AR}$ such that $\perp<\delta^{\mathrm{A}}<\alpha^{\mathrm{A}}$. Suppose that $\mathrm{AR}_{s} \cup \mathrm{AR}_{u}$ is not a conflict-free set. Thus, by Definition 14 we have that $\exists \mathrm{A}, \mathrm{B} \in \mathrm{AR}_{s}$ $\cup \mathrm{AR}_{u}$ such that $(\mathrm{B}, \mathrm{A}) \in \mathcal{R}_{a}$ with $\mathrm{B} \succ \mathrm{A}$. By hypothesis, the unique possible valuation for the arguments are $\delta^{\mathrm{B}}=\top$ and $\delta^{\mathrm{A}}=\top$. However, if $(\mathrm{B}, \mathrm{A}) \in \mathcal{R}_{a}$, then by Definition 8 and Definition 11 we know that $\delta^{\mathrm{A}}=\perp$. Contradiction, since by Definition 13, we know that if $\delta^{\mathrm{A}}=\perp$, then $\mathrm{A} \in \mathrm{AR}_{n}$. Thus, for this reason $\mathrm{A} \notin \mathrm{AR}_{s} \cup \mathrm{AR}_{u}$. Therefore, there not exist two arguments $\mathrm{A}, \mathrm{B} \in \mathrm{AR}_{s} \cup \mathrm{AR}_{u}$ verifying that $(\mathrm{B}, \mathrm{A}) \in \mathcal{R}_{a}$. Thus, we can conclude that $\mathrm{AR}_{s} \cup \mathrm{AR}_{u}$ is conflict-free.

Theorem 3 Let $\Psi=\langle\Theta, \mathbf{A}, v\rangle$ be an $L-B A F$, and $G \Psi$ be the corresponding labeled bipolar argumentation graph. If all arguments $\mathrm{A} \in G$ are such that $\alpha^{\mathrm{A}}=\top$ when $(\overrightarrow{\mathrm{A}})=\emptyset$, and $\alpha^{\mathrm{A}}=\perp$ when $(\mathrm{A}) \neq \emptyset$, then

(i) $\mathrm{AR}_{u} \cup \mathrm{AR}_{s}$ is the d-preferred extension;

(ii) If $\mathrm{AR}_{s} \cup \mathrm{AR}_{u}$ is safe, then $\mathrm{AR}_{s} \cup \mathrm{AR}_{u}$ is the s-preferred extension; and

(iii) If $\mathrm{AR}_{s} \cup \mathrm{AR}_{u}$ is closed under $\mathcal{R}_{s}$, then $\mathrm{AR}_{s} \cup \mathrm{AR}_{u}$ is the c-preferred extension.

Proof: We separate the proof in three parts:

(i) $\mathrm{AR}_{s} \cup \mathrm{AR}_{u}$ is the d-preferred extension. By Proposition 5-(ii) we know that $\mathrm{AR}_{s} \cup \mathrm{AR}_{u}$ is conflict-free set. Furthermore, by Proposition 2 we know that $\mathrm{AR}_{s} \cup \mathrm{AR}_{u} \cup \mathrm{AR}_{w} \cup \mathrm{AR}_{n}=\mathrm{AR}$, which is a finite cover of AR. In addition, by hypothesis and Proposition 5-(i), we can say that $\mathrm{AR}_{w}=\emptyset$, then $\mathrm{AR}_{s} \cup \mathrm{AR}_{u} \cup \mathrm{AR}_{n}=\mathrm{AR}$. Thus, $\mathrm{AR}_{s} \cup \mathrm{AR}_{u}$ is the maximal conflict-free set that defends all its elements in $\Psi$. Now, we need to prove that $\mathrm{AR}_{S} \cup \mathrm{AR}_{u}=S$ where $S$ is the d-preferred extension in the underlying bipolar argumentation framework $\Theta$.

- Suppose that $\mathrm{AR}_{S} \cup \mathrm{AR}_{u} \nsubseteq S$, then there exists an argument $\mathrm{A} \in \mathrm{AR}_{S} \cup \mathrm{AR}_{u}$ and $\mathrm{A} \notin S$. Thus, by Definition 5 there exists an argument $B \in A R$ such that $B$ is a supported or secondary defeat of $A$ and there does not exist an argument $C \in S$ such that $C$ is a supported or secondary defeat of $\mathrm{B}$. However, by hypothesis we have that $\mathrm{A} \in \mathrm{AR}_{s} \cup \mathrm{AR}_{u}$, then the following cases can occur:

If $\mathrm{A} \in \mathrm{AR}_{u}$, then $\alpha^{\mathrm{A}}=\delta^{\mathrm{A}}=\top$. Then, can arise one of the following cases:

$\overrightarrow{(A)}=\emptyset$ reflecting that the argument A has not any attacker in AR. Thus, by Definition 6 A must belong to $S$. Contradiction.

$\overrightarrow{(A)} \neq \emptyset$ reflecting that any attacker of $A$ is neutralized. Contradiction, by Definition 6 A must belong to $S$. 
If $\mathrm{A} \in \mathrm{AR}_{s}$, then $\alpha^{\mathrm{A}}=\perp$ and $\delta^{\mathrm{A}}=\top$. Then, can arise one of the following cases:

$\overrightarrow{(A)}=\emptyset$ reflecting that the argument $A$ has not any attacker in AR, and by Definition 11 any argument $\mathrm{A}_{j} \in \overrightarrow{(\mathrm{A})}$ satisfies that $\delta^{\mathrm{A}_{j}}=\top$. Thus, by Definition $6 \mathrm{~A}$ must belong to $S$. Contradiction.

$\overrightarrow{(A)} \neq \emptyset$ reflecting that any attacker of A is neutralized. Thus, by Definition 6 A must belong to $S$. Contradiction.

- Suppose that $S \nsubseteq \mathrm{AR}_{S} \cup \mathrm{AR}_{u}$, then there exists an argument A such that $\mathrm{A} \in S$ and $\mathrm{A} \notin$ $\mathrm{AR}_{s} \cup \mathrm{AR}_{u}$. If $\mathrm{A} \notin \mathrm{AR}_{s} \cup \mathrm{AR}_{u}$, then $\delta^{\mathrm{A}}=\perp$. Thus, we must analyze the following cases:

If $\delta^{\mathrm{A}}=\perp$ and $\overrightarrow{(\mathrm{A})}=\emptyset$, then $\mu^{\mathrm{A}}=\alpha^{\mathrm{A}}=\top$. Thus, by Definition 11 there exists an argument $\mathrm{X}_{l} \in \overrightarrow{(\mathrm{A})}$ satisfying that $\delta^{\mathrm{x}_{l}}=\top$ with $l \in\{1, \ldots, n\}$. However, by hypothesis, $A \in S$. Then, by Definition 5, for any argument $B \in A R$ such that $B$ is a supported or secondary defeat of $\mathrm{A}$, there exists an argument $\mathrm{C} \in S$ such that $\mathrm{C}$ is a supported or secondary defeat of $\mathrm{B}$. Thus, $\delta^{\mathrm{A}}=\top$ then $\mathrm{A} \in \mathrm{AR}_{s}$. Contradiction.

If $\delta^{\mathrm{A}}=\perp$ and $\overrightarrow{(\mathrm{A})} \neq \emptyset$, then $\alpha^{\mathrm{A}}=\perp$. Furthermore, by Definition 11 there exists an argument $\mathrm{A}_{l} \in \overrightarrow{(\mathrm{A})}$ with $l \in\{1, \ldots, m\}$, satisfying that $\delta^{\mathrm{A}_{l}}=\perp$. Thus, there exists an attacker $\mathrm{X}_{t}$ for $\mathrm{A}_{l}$ verfying that $\delta^{\mathrm{x}_{k}}=T$. However, by hypothesis $\mathrm{A} \in S$, then by Definition 5 for any argument $B \in A R$ such that $B$ is a supported or secondary defeat of $\mathrm{A}$ and there exists an argument $\mathrm{C} \in S$ such that $\mathrm{C}$ is a supported or secondary defeat of B. Thus, $\delta^{\mathrm{A}}=\top$, then $\mathrm{A} \in \mathrm{AR}_{s}$. Contradiction.

(ii) If $\mathrm{AR}_{s} \cup \mathrm{AR}_{u}$ is safe, then $\mathrm{AR}_{s} \cup \mathrm{AR}_{u}$ is the s-preferred extension. $\mathrm{By}(i)$ we know that $\mathrm{AR}_{s} \cup$ $\mathrm{AR}_{u}$ is the d-preferred extension in $\Theta$. That is, $\mathrm{AR}_{s} \cup \mathrm{AR}_{u}$ is the maximal conflict-free set that defends all its elements. In addition, by hypothesis we know that $\mathrm{AR}_{s} \cup \mathrm{AR}_{u}$ is safe. Thus, $\mathrm{AR}_{s}$ $\cup \mathrm{AR}_{u}$ is the maximal conflict free and safe set that defends all its elements, corresponding to the s-preferred extension (Definition 7).

(iii) If $\mathrm{AR}_{s} \cup \mathrm{AR}_{u}$ is closed under $\mathcal{R}_{s}$, then $\mathrm{AR}_{s} \cup \mathrm{AR}_{u}$ is the c-preferred extension. $\mathrm{By}(i)$ we know that $\mathrm{AR}_{s} \cup \mathrm{AR}_{u}$ is the d-preferred extension in $\Theta$. That is, $\mathrm{AR}_{s} \cup \mathrm{AR}_{u}$ is the maximal conflict-free set that defends all its elements. In addition, by hypothesis we know that $\mathrm{AR}_{s} \cup \mathrm{AR}_{u}$ is closed under $\mathcal{R}_{s}$. Thus, $\mathrm{AR}_{s} \cup \mathrm{AR}_{u}$ is the maximal conflict free closed under $\mathcal{R}_{s}$ set that defends all its elements, corresponding to the c-preferred extension (Definition 7).

Proposition 6 Let $\Psi=\langle\Theta, \mathbf{A}, v\rangle$ be an $L-B A F$, and $\Omega_{\Psi}^{\mathrm{q}}$ and $\Omega_{\Psi}^{\mathrm{s}}$ be the general effectiveness support and quality coefficients associated with $\Psi$, respectively. We have that: if $\Omega_{\Psi}^{\mathrm{q}}=1$, then $\Omega_{\Psi}^{\mathrm{s}}=0$.

Proof: By hypothesis $\Omega_{\Psi}^{q}=1$. Thus, by Definition 17, we have that $\Omega_{\Psi}^{q}=\frac{\sum_{\mathbf{A} \in \mathbf{A}} \omega_{X}^{q}}{\# \mathbf{A}}=1$ with \#A $=n$. Hence, we have that $\sum_{\mathrm{A} \in \mathbf{A}} \omega_{\mathrm{X}}^{\mathrm{q}}$ must be equal to $n$. Furthermore, by Definition 17, we know that $\omega_{\mathrm{X}}^{\mathrm{q}}$ is defined as $\frac{\sum_{\mathrm{A} \in \mathrm{AR}} \alpha_{\mathrm{X}}^{\mathrm{A}}}{\# \mathrm{AR}}$. Then, in order to satisfy the last condition, $\frac{\sum_{\mathrm{A} \in \mathrm{AR}} \alpha_{\mathrm{X}}^{\mathrm{A}}}{\# \mathrm{AR}}$ must be equal to 1. By Lemma 1, we know that $\alpha^{\mathrm{A}} \in[\perp, \top]$ (in particular the interval $[0,1]$ ). Thus, $\frac{\sum_{\mathrm{A} \in \mathrm{AR}} \alpha_{\mathrm{X}}^{\mathrm{A}}}{\# \mathrm{AR}}=1$, 
if $\alpha_{\mathrm{X}}^{\mathrm{A}}=1$ for each argument $\mathrm{A} \in \mathrm{AR}$. Hence, by Lemma $2-(i)$ we know that $\mu_{\mathrm{X}}^{\mathrm{A}} \geq \alpha_{\mathrm{X}}^{\mathrm{A}}$. Then, we can conclude that $\mu_{\mathrm{X}}^{\mathrm{A}}=\alpha_{\mathrm{X}}^{\mathrm{A}}=1$. In another hand, by Definition 17 we know that $\Omega_{\Psi}^{\mathrm{s}}=\frac{\sum_{\mathrm{A} \in \mathbf{A}} \omega_{\mathrm{X}}^{\mathrm{S}}}{\# \mathbf{A}}$, where $\omega_{\mathrm{X}}^{\mathrm{s}}=\frac{\sum_{\mathrm{A} \in \mathrm{AR}} \mu_{\mathrm{X}}^{\mathrm{A}}-\alpha_{\mathrm{X}}^{\mathrm{A}}}{\# \mathcal{R}_{s}}$. Furthermore, by hypothesis we show that $\mu_{\mathrm{X}}^{\mathrm{A}}=\alpha_{\mathrm{X}}^{\mathrm{A}}$. Thus, we can affirm that $\mu_{\mathrm{X}}^{\mathrm{A}}-\alpha_{\mathrm{X}}^{\mathrm{A}}=0$ for each argument $\mathrm{A} \in \mathrm{AR}$. Finally, we can deduce that $\omega_{\mathrm{X}}^{\mathrm{s}}=\frac{\sum_{\mathrm{A} \in \mathrm{AR}} \mu_{\mathrm{X}}^{\mathrm{A}}-\alpha_{\mathrm{X}}^{\mathrm{A}}}{\# \mathcal{R}_{s}}=0$, and for that $\Omega_{\Psi}^{\mathrm{s}}=\frac{\sum_{\mathrm{A} \in \mathbf{A}} \omega_{\mathrm{X}}^{\mathrm{s}}}{\# \mathbf{A}}=0$.

Proposition 7 Let $\Psi=\langle\Theta, \mathbf{A}, v\rangle$ be an $L-B A F, \Omega_{\Psi}^{\mathrm{q}}$ be the general quality coefficient associated with $\Psi$, and $\mathrm{AR}_{w}$ and $\mathrm{AR}_{s}$ be the sets of weakened and strengthened arguments associated with $\Psi$, respectively. We have that, if $\Omega_{\Psi}^{\mathrm{q}}=1$, then $\mathrm{AR}_{w}=\emptyset$ and $\mathrm{AR}_{s}=\emptyset$.

Proof: By the hypothesis and Definition 17 we know that $\Omega_{\Psi}^{\mathrm{q}}=\frac{\sum_{\mathbf{A} \in \mathbf{A}} \omega_{X}^{\mathrm{q}}}{\# \mathbf{A}}=1$ with $\# \mathbf{A}=n$. Hence, we have that $\sum_{\mathrm{A} \in \mathbf{A}} \omega_{X}^{\mathrm{q}}$ must be equal to $n$. Furthermore, by Definition 17, we know that $\omega_{X}^{\mathrm{q}}$ is defined as $\frac{\sum_{\mathrm{A} \in \mathrm{AR}} \alpha_{\mathrm{X}}^{\mathrm{A}}}{\text { \#AR }}$. Then, in order to satisfy the last condition, $\frac{\sum_{\mathrm{A} \in \mathrm{AR}} \alpha_{\mathrm{X}}^{\mathrm{A}}}{\text { \#AR }}$ must be equal to 1. By Lemma 1, we know that $\alpha_{X}^{\mathrm{A}} \in[\perp, \top]$ (in particular the interval $[0,1]$ ). Thus, $\frac{\sum_{\mathrm{A} \in \mathrm{AR}} \alpha_{\mathrm{X}}^{\mathrm{A}}}{\# \mathrm{AR}}=1$, if $\alpha_{\mathrm{X}}^{\mathrm{A}}=1$ for each argument $\mathrm{A} \in \mathrm{AR}$. Hence, by Lemma 2-(ii) we know that $\mu_{\mathrm{X}}^{\mathrm{A}} \geq \alpha_{\mathrm{X}}^{\mathrm{A}}$. Then, we can conclude that $\mu_{\mathrm{X}}^{\mathrm{A}}=\alpha_{\mathrm{X}}^{\mathrm{A}}=1$. Now, we will prove that for any argument $\mathrm{A} \in \mathrm{AR}$ verifies that $\delta_{\mathrm{X}}^{\mathrm{A}} \in\{0,1\}$.

(i) If $\overrightarrow{(\mathrm{A})}=\emptyset$, then by Definition $11 \delta_{\mathrm{X}}^{\mathrm{A}}=\mu_{\mathrm{X}}^{\mathrm{A}}$. Thus, we can deduce that $\delta_{\mathrm{X}}^{\mathrm{A}}=1$.

(ii) If $\overrightarrow{(\mathrm{A})} \neq \emptyset$ and $\overrightarrow{(\mathrm{A})}=\emptyset$, we must analyze two possibilities:

- If there exists an argument $\mathrm{B} \in \overrightarrow{(\mathrm{A})}$ such that verifies that $\delta_{\mathrm{X}}^{\mathrm{B}}=1$, then by Definition 11 and Definition 8 we know that $\delta_{\mathrm{X}}^{\mathrm{A}}=0$.

- If any argument $\mathrm{X}_{k} \in \overrightarrow{(\mathrm{A})}$ verifies that $\delta_{\mathrm{X}}^{\mathrm{X}_{\mathrm{K}}}=0$, then by Definition 11 and Definition 8 we have that $\delta_{\mathrm{X}}^{\mathrm{A}}=\alpha_{\mathrm{X}}^{\mathrm{A}}=1$.

(iii) If $\overrightarrow{(A)} \neq \emptyset$ and $\overrightarrow{(A)} \neq \emptyset$, by Definition 11 we must analyze two possibilities:

- If $\otimes_{k=1}^{n} \delta_{\mathrm{X}}^{\mathrm{X}_{k}} \leq \odot_{j=1}^{m} \delta_{\mathrm{X}}^{\mathrm{A}_{j}}$ with $\mathrm{X}_{k} \in \overrightarrow{(\mathrm{A})}$ and $\mathrm{A}_{j} \in(\overrightarrow{\mathrm{A}})$, then by Definition 11 we have that $\delta_{\mathrm{X}}^{\mathrm{A}}=$ $\alpha_{\mathrm{X}}^{\mathrm{A}} \oplus\left(\odot_{j=1}^{m} \delta_{\mathrm{X}}^{\mathrm{A}_{j}} \ominus \otimes_{k=1}^{n} \delta_{\mathrm{X}}^{\mathrm{X}_{k}}\right)$. However, like $\alpha_{\mathrm{X}}^{\mathrm{A}}=1$ by Definition 8 we must conclude that $\delta_{\mathrm{X}}^{\mathrm{A}}=1$.

- If $\otimes_{k=1}^{n} \delta_{\mathrm{X}}^{\mathrm{X}_{k}}>\odot_{j=1}^{m} \delta_{\mathrm{X}}^{\mathrm{A}_{j}}$, with $\mathrm{X}_{k} \in \overrightarrow{(\mathrm{A})}$ and $\mathrm{A}_{j} \in \overrightarrow{(\mathrm{A})}$, then by Definition 11 we have that $\delta_{\mathrm{X}}^{\mathrm{A}}=\alpha_{\mathrm{X}}^{\mathrm{A}} \ominus\left(\otimes_{k=1}^{n} \delta_{\mathrm{X}}^{\mathrm{X}_{k}} \ominus \odot_{j=1}^{m} \delta_{\mathrm{X}}^{\mathrm{A}_{j}}\right)$. We need to have an argument $\mathrm{X}_{l} \in \overrightarrow{(\mathrm{A})}$ such that satisfies that $\delta_{\mathrm{X}}^{\mathrm{X}_{l}}=1$, with $l \in\{1, \ldots, n\}$. It is a consequence of if any argument $\mathrm{X}_{k} \in \overrightarrow{(\mathrm{A})}$ verifies that $\delta_{\mathrm{X}}^{\mathrm{X}_{k}}=0$, then $\otimes_{k=1}^{n} \delta_{\mathrm{X}}^{\mathrm{X}_{k}}=0$. Contradiction. Hence, there must exist an argument $\mathrm{X}_{l} \in \overrightarrow{(\mathrm{A})}$ such that $\delta_{\mathrm{X}}^{\mathrm{X}_{l}}=1$; thus $\odot_{k=1}^{n} \delta_{\mathrm{X}}^{\mathrm{X}_{k}}=1$. Furthermore, there exists an argument 
$\mathrm{A}_{j} \in \overrightarrow{(\mathrm{A})}$ that must verify that $\delta_{\mathrm{X}}^{\mathrm{A}_{j}}=0$, thus $\odot_{j=1}^{m} \delta_{\mathrm{X}}^{\mathrm{A}_{j}}=0$. Finally, by Definition 11 we have that $\delta_{\mathrm{X}}^{\mathrm{A}}=0$.

Finally, we can conclude that $\delta_{\mathrm{X}}^{\mathrm{A}}=1$ or $\delta_{\mathrm{X}}^{\mathrm{A}}=0$. Then, by Definition 13 the status assigned to $\mathrm{A}$ is unchallenged in case that $\delta_{\mathrm{X}}^{\mathrm{A}}=1$ or neutralized in case that $\delta_{\mathrm{X}}^{\mathrm{A}}=0$. In this direction, we must affirm that $\mathrm{AR}_{w}=\emptyset$ and $\mathrm{AR}_{s}=\emptyset$.

Proposition 8 Let $\Psi=\langle\Theta, \mathbf{A}, v\rangle$ be an $L-B A F$ and $\Psi^{\prime}=\left\langle\Theta^{\prime}, \mathbf{A}, v\right\rangle$ be the pruned labeled bipolar argumentation framework based on $\Psi$. Then, the relation between the general quality of arguments satisfies that $\Omega_{\Psi}^{\mathrm{q}} \leq \Omega_{\Psi^{\prime}}^{\mathrm{q}}$.

Proof:

By hypothesis we have that $\Psi=\langle\Theta, \mathbf{A}, v\rangle$ is an $L-B A F$ and $\Psi^{\prime}=\left\langle\Theta^{\prime}, \mathbf{A}, v\right\rangle$ is the refined $L-B A F$ obtained after to apply the pruning process presented in Definition 18. Hence, by Definition 17, we have that $\Omega_{\Psi}^{\mathrm{q}}=\frac{\sum_{\mathrm{A} \in \mathbf{A}} \omega_{\mathrm{X}}^{\mathrm{q}}}{\# \mathbf{A}}$ and $\omega_{X}^{\mathrm{q}}=\frac{\sum_{\mathrm{A} \in \mathrm{AR}} \alpha_{X}^{\mathrm{A}}}{\# \text { AR }}$. Since $\Omega_{\Psi}^{\mathrm{q}}$ and $\omega_{X}^{\mathrm{q}}$ are the arithmetic mean that obtain the value based on the sum of a collection of numbers divided by the count of numbers in the collection, then we have that $\min \left(\alpha_{X}^{\mathrm{A}}\right) \leq \omega_{\mathrm{X}}^{\mathrm{q}} \leq \max \left(\alpha_{\mathrm{X}}^{\mathrm{A}}\right)$ with $\mathrm{A} \in \mathrm{AR}$. However, by Definition 18, $\mathrm{AR}^{\prime}$ is the set of relevant arguments in $\mathrm{AR}\left(\mathrm{AR}^{\prime}:=\left\{\mathrm{A} \in \mathrm{AR} \mid \alpha_{X}^{\mathrm{A}} \geq \omega_{\mathrm{X}}^{\mathrm{q}}\right.\right.$ for each $\left.\left.\left.i=1, \ldots n\right\}\right)\right)$. Thus, we can deduce that $\omega_{\mathrm{X}}^{\mathrm{q}} \leq \min \left(\alpha_{\mathrm{X}}^{\mathrm{A}}\right) \leq \omega_{\mathrm{X}}^{\mathrm{q} \prime} \leq \max \left(\alpha_{\mathrm{X}}^{\mathrm{A}}\right)$ with $\mathrm{A} \in \mathrm{AR}^{\prime}$. Thus, we can say that $\omega_{\mathrm{X}}^{\mathrm{q}^{\prime}} \geq \omega_{\mathrm{X}}^{\mathrm{q}}$. Finally, we deduce that $\Omega_{\Psi}^{q} \leq \Omega_{\Psi}^{q \prime}$.

Theorem 4 Any L-BAF instantiation satisfies the following postulates: Abstraction, Independence, Monotony, Directionality, Equivalence, Quality Precedence, Neutrality, Reinforcement, Stability, Weakening, Strengthening, Causality, Neutralization, and Proportionality.

Proof: In order to prove this theorem, we analyze each postulate in the context of $L-B A F$, corroborating that they are satisfied. In each case, we are assuming the corresponding context represented by the $L-B A F \Psi=\langle\Theta, \mathbf{A}, v\rangle$ and its underlying $B A F \Theta=\left\langle\mathrm{AR}, \mathcal{R}_{a}, \mathcal{R}_{s}\right\rangle$.

Abstraction. Given two L-BAFs $\Psi=\langle\Theta, \mathbf{A}, v\rangle$ and $\Psi^{\prime}=\left\langle\Theta^{\prime}, \mathbf{A}^{\prime}, v^{\prime}\right\rangle$, and an isomorphism $h$ : $\Psi \longrightarrow \Psi^{\prime}$, then the following property holds:

For all $\mathrm{A} \in \mathrm{AR},\left\langle\alpha^{\mathrm{A}}, \mu^{\mathrm{A}}, \delta^{\mathrm{A}}\right\rangle \in \Lambda_{\Psi}$ if and only if $\left\langle\alpha^{h(\mathrm{~A})}, \mu^{h(\mathrm{~A})}, \delta^{h(\mathrm{~A})}\right\rangle \in \Lambda_{\Psi^{\prime}}$

By Definition 11, the original valuations are given as $\alpha^{\mathrm{A}}=\rho(\mathrm{A})$ and $\alpha^{h(\mathrm{~A})}=\rho^{\prime}(h(\mathrm{~A}))$, and by hypothesis $h$ is an isomorphism from $\Psi$ to $\Psi^{\prime}$ so by Definition 16 we know that $v(\mathrm{~A})=v^{\prime}(h(\mathrm{~A}))$. Thus $\alpha^{\mathrm{A}}=\alpha^{h(\mathrm{~A})}$. Furthermore, by Definition 16 we have that $\overrightarrow{(\mathrm{A})} \simeq(\overrightarrow{h(\mathrm{~A})})$ and $(\overrightarrow{\mathrm{A}}) \simeq(h(\mathrm{~A}))$. That is, if $\mathrm{X}_{k} \in \overrightarrow{(\mathrm{A})}$ then $h\left(\mathrm{X}_{\mathrm{k}}\right) \in \overrightarrow{(\mathrm{A})}$, and if $\mathrm{A}_{j} \in \overrightarrow{(\mathrm{A})}$ then $h\left(\mathrm{~A}_{\mathrm{j}}\right) \in \overrightarrow{(\mathrm{A})}$. Hence, we can see that there exists the following bijective correspondence $\delta^{\mathrm{X}_{k}} \mapsto \delta^{h\left(\mathrm{X}_{\mathrm{k}}\right)}$ and $\delta^{\mathrm{A}_{j}} \mapsto \delta^{h\left(\mathrm{~A}_{\mathrm{j}}\right)}$, and consequently $\otimes_{k=1}^{n} \delta^{\mathrm{X}_{\mathrm{k}}} \mapsto$ $\otimes_{k=1}^{n} \delta^{h\left(\mathrm{X}_{\mathrm{k}}\right)}$ and $\odot_{j=1}^{m} \delta^{\mathrm{A}_{\mathrm{j}}} \mapsto \odot_{j=1}^{m} \delta^{h\left(\mathrm{~A}_{\mathrm{j}}\right)}$. Hence $\mu^{\mathrm{A}} \mapsto \mu^{h(\mathrm{~A})}$ and $\delta^{\mathrm{A}} \mapsto \delta^{h(\mathrm{~A})}$. Finally, for the above and Definition 11 we can conclude that there exists the following bijective correspondence $\Lambda_{\Psi} \rightarrow$ $\Lambda_{\Psi^{\prime}} /\left\langle\alpha^{\mathrm{A}}, \mu^{\mathrm{A}}, \delta^{\mathrm{A}}\right\rangle \mapsto\left\langle\alpha^{h(\mathrm{~A})}, \mu^{h(\mathrm{~A})}, \delta^{h(\mathrm{~A})}\right\rangle$. 
INDEPENDENCE. Let $\mathrm{A}$ and $\mathrm{B}$ be two arguments in $\mathrm{AR}$ that are not connected in $\mathrm{G}_{\Theta}$. Then, the valuations $\left\langle\alpha^{\mathrm{A}}, \mu^{\mathrm{A}}, \delta^{\mathrm{A}}\right\rangle,\left\langle\alpha^{\mathrm{B}}, \mu^{\mathrm{B}}, \delta^{\mathrm{B}}\right\rangle \in \lambda(\Theta)$ associated with $\mathrm{A}$ and $\mathrm{B}$, respectively, are independent.

By Definition 11, the original valuations are given as follows: $\alpha^{\mathrm{A}}=\rho(\mathrm{A})$ and $\alpha^{\mathrm{B}}=\rho(\mathrm{B})$. Hence, the original valuations are independent. Thus, we must analyze the strengthened and overall valuations associated with the arguments involved in the graph $\mathrm{G}_{\Theta}$. Analyzing Definition 11, the strengthened and overall valuation associated with an argument are affected by its corresponding supports and attackers, respectively.

Furthermore, by hypothesis $B$ is not connected with $A$ in $G_{\Theta}$, then $B \notin \overrightarrow{(A)}, B \notin \overrightarrow{(A)}$ and vice versa. This leads us to conclude that the valuations associated with the arguments $A$ and $B$ are independent.

Monotony. Let $\left\langle\alpha^{\mathrm{A}}, \mu^{\mathrm{A}}, \delta^{\mathrm{A}}\right\rangle,\left\langle\alpha^{\mathrm{B}}, \mu^{\mathrm{B}}, \delta^{\mathrm{B}}\right\rangle \in \lambda(\Theta)$ be the valuations associated with two arguments $\mathrm{A}$ and $\mathrm{B}$ respectively. It holds that if

(i) $\alpha^{\mathrm{A}}=\alpha^{\mathrm{B}}$,

(ii) $\odot_{j=1}^{m} \delta^{\mathrm{A}_{j}} \geq \odot_{j=1}^{r} \delta^{\mathrm{B}_{j}}$ with $\mathrm{A}_{j} \in \overrightarrow{(\mathrm{A})}, 1 \leq j \leq m$, and $\mathrm{B}_{j} \in \overrightarrow{(\mathrm{B})}, 1 \leq j \leq r$,

(iii) $\otimes_{k=1}^{n} \delta^{\mathrm{x}_{k}} \leq \otimes_{k=1}^{s} \delta^{\mathrm{Y}_{k}}$ with $\mathrm{X}_{k} \in \overrightarrow{(\mathrm{A})}, 1 \leq k \leq n$, and $\mathrm{Y}_{k} \in \overrightarrow{(\mathrm{B})}, 1 \leq k \leq s$,

then $\delta^{\mathrm{A}} \geq \delta^{\mathrm{B}}$.

By Definition 11, we analyze how the overall valuations for A and B are obtained in $L A F$.

- When $\otimes_{k=1}^{n} \delta^{\mathrm{X}_{k}} \leq \odot_{j=1}^{m} \delta^{\mathrm{A}_{j}}$ with $\mathrm{X}_{k} \in \overrightarrow{(\mathrm{A})}$ and $\mathrm{A}_{j} \in \overrightarrow{(\mathrm{A})}$, and $\otimes_{k=1}^{s} \delta^{\mathrm{Y}_{k}} \leq \odot_{j=1}^{r} \delta^{\mathrm{B}_{j}}$ with $\mathrm{Y}_{k} \in \overrightarrow{(\mathrm{B})}$ and $\mathrm{B}_{j} \in(\mathrm{B})$. We have that $\delta^{\mathrm{A}}=\alpha^{\mathrm{A}} \oplus\left(\odot_{j=1}^{m} \delta^{\mathrm{A}_{j}} \ominus \otimes_{k=1}^{n} \delta^{\mathrm{x}_{k}}\right)$ and $\delta^{\mathrm{B}}=\alpha^{\mathrm{B}} \oplus\left(\odot_{j=1}^{r} \delta^{\mathrm{B}_{j}} \ominus\right.$ $\left.\otimes_{k=1}^{s} \delta^{\mathrm{Y}_{k}}\right)$. By hypothesis, we know that $\left(\odot_{j=1}^{m} \delta^{\mathrm{A}_{j}}\right) \geq\left(\odot_{j=1}^{r} \delta^{\mathrm{B}_{j}}\right)$ with $\mathrm{A}_{j} \in(\overrightarrow{\mathrm{A}})$ and $\mathrm{B}_{j} \in \overrightarrow{(\mathrm{B})}$, and $\otimes_{k=1}^{n} \delta^{\mathrm{X}_{k}} \leq \otimes_{k=1}^{s} \delta^{\mathrm{Y}_{k}}$ with $\mathrm{X}_{k} \in \overrightarrow{(\mathrm{A})}$ and $\mathrm{Y}_{k} \in \overrightarrow{(\mathrm{B})}$. Thus, we have that $\odot_{j=1}^{m} \delta^{\mathrm{A}_{j}} \ominus \otimes_{k=1}^{n} \delta^{\mathrm{X}_{k}} \geq$ $\odot_{j=1}^{r} \delta^{\mathrm{B}_{j}} \ominus \otimes_{k=1}^{s} \delta^{\mathrm{Y}_{k}}$. Furthermore, by hypothesis we know that $\alpha^{\mathrm{A}}=\alpha^{\mathrm{B}}$, then $\delta^{\mathrm{A}} \geq \delta^{\mathrm{B}}$.

- When $\otimes_{k=1}^{n} \delta^{\mathrm{X}_{k}}>\odot_{j=1}^{m} \delta^{\mathrm{A}_{j}}$ with $\mathrm{X}_{k} \in \overrightarrow{(\mathrm{A})}$ and $\mathrm{A}_{j} \in(\overrightarrow{\mathrm{A}})$, and $\otimes_{k=1}^{s} \delta^{\mathrm{Y}_{k}}>\odot_{j=1}^{r} \delta^{\mathrm{B}_{j}}$ with $\mathrm{Y}_{k} \in \overrightarrow{(\mathrm{B})}$ and $\mathrm{B}_{j} \in(\mathrm{B})$. We have that $\delta^{\mathrm{A}}=\alpha^{\mathrm{A}} \ominus\left(\otimes_{k=1}^{n} \delta^{\mathrm{x}_{k}} \ominus \odot_{j=1}^{m} \delta^{\mathrm{A}_{j}}\right)$ and $\delta^{\mathrm{B}}=\alpha^{\mathrm{B}} \ominus\left(\otimes_{k=1}^{s} \delta^{\mathrm{Y}_{k}} \ominus\right.$ $\left.\odot_{j=1}^{r} \delta^{\mathrm{B}_{j}}\right)$, with $\mathrm{X}_{k} \in \overrightarrow{(\mathrm{A})}$ and $\mathrm{A}_{j} \in(\overrightarrow{\mathrm{A}})$, and $\mathrm{Y}_{k} \in \overrightarrow{(\mathrm{B})}$ and $\mathrm{B}_{j} \in \overrightarrow{(\mathrm{B})}$. By hypothesis, we have that $\left(\odot_{j=1}^{m} \delta^{\mathrm{A}_{j}}\right) \geq\left(\odot_{j=1}^{r} \delta^{\mathrm{B}_{j}}\right)$ with $\mathrm{A}_{j} \in(\overrightarrow{\mathrm{A}})$ and $\mathrm{B}_{j} \in(\overrightarrow{\mathrm{B}})$, and $\left(\otimes_{k=1}^{n} \delta^{\mathrm{X}_{k}}\right) \leq\left(\otimes_{k=1}^{s} \delta^{\mathrm{Y}_{k}}\right)$ with $\mathrm{X}_{k} \in \overrightarrow{(\mathrm{A})}$ and $\mathrm{Y}_{k} \in \overrightarrow{(\mathrm{B})}$. Thus, we have that $\otimes_{k=1}^{n} \delta^{\mathrm{X}_{k}} \ominus \odot_{j=1}^{m} \delta^{\mathrm{A}_{j}} \leq \otimes_{k=1}^{s} \delta^{\mathrm{Y}_{k}} \ominus \odot_{j=1}^{r} \delta^{\mathrm{B}_{j}}$. Furthermore, by hypothesis we have that $\alpha^{\mathrm{A}}=\alpha^{\mathrm{B}}$, then $\delta^{\mathrm{A}} \geq \delta^{\mathrm{B}}$.

- When $\otimes_{k=1}^{n} \delta^{\mathrm{X}_{k}} \leq \odot_{j=1}^{m} \delta^{\mathrm{A}_{j}}$ with $\mathrm{X}_{k} \in \overrightarrow{(\mathrm{A})}$ and $\mathrm{A}_{j} \in \overrightarrow{(\mathrm{A})}$, and $\otimes_{k=1}^{s} \delta^{\mathrm{Y}_{k}}>\odot_{j=1}^{r} \delta^{\mathrm{B}_{j}}$ with $\mathrm{Y}_{k} \in \overrightarrow{(\mathrm{B})}$ and $\mathrm{B}_{j} \in(\mathrm{B})$. We have that $\delta^{\mathrm{A}}=\alpha^{\mathrm{A}} \oplus\left(\odot_{j=1}^{m} \delta^{\mathrm{A}_{j}} \ominus \otimes_{k=1}^{n} \delta^{\mathrm{x}_{k}}\right)$ and $\delta^{\mathrm{B}}=\alpha^{\mathrm{B}} \ominus\left(\otimes_{k=1}^{s} \delta^{\mathrm{Y}_{k}} \ominus\right.$ $\left.\odot_{j=1}^{r} \delta^{\mathrm{B}_{j}}\right)$. By hypothesis, we know that $\alpha^{\mathrm{A}}=\alpha^{\mathrm{B}}$. Thus, we conclude that $\delta^{\mathrm{A}} \geq \delta^{\mathrm{B}}$ since $\otimes_{k=1}^{s} \delta^{\mathrm{Y}_{k}} \ominus \odot_{j=1}^{r} \delta^{\mathrm{B}_{j}}>\perp$ with $\mathrm{Y}_{k} \in \overrightarrow{(\mathrm{B})}$ and $\mathrm{B}_{j} \in \overrightarrow{(\mathrm{B})}$. 
- When $\otimes_{k=1}^{n} \delta^{\mathrm{X}_{k}}>\odot_{j=1}^{m} \delta^{\mathrm{A}_{j}}$ with $\mathrm{X}_{k} \in \overrightarrow{(\mathrm{A})}$ and $\mathrm{A}_{j} \in \overrightarrow{(\mathrm{A})}$, and $\otimes_{k=1}^{s} \delta^{\mathrm{Y}_{k}} \leq \odot_{j=1}^{r} \delta^{\mathrm{B}_{j}}$ with $\mathrm{Y}_{k} \in \overrightarrow{(\mathrm{B})}$ and $\mathrm{B}_{j} \in \overrightarrow{(\mathrm{B})}$. By hypothesis, we know $\odot_{j=1}^{m} \delta^{\mathrm{A}_{j}} \geq \odot_{j=1}^{r} \delta^{\mathrm{B}_{j}}$ then $\otimes_{k=1}^{s} \delta^{\mathrm{Y}_{k}} \leq \odot_{j=1}^{r} \delta^{\mathrm{B}_{j}} \leq$ $\odot_{j=1}^{m} \delta^{\mathrm{A}_{j}}<\otimes_{k=1}^{n} \delta^{\mathrm{x}_{k}}$, with $\mathrm{A}_{j} \in(\overrightarrow{\mathrm{A}}), \mathrm{B}_{j} \in\left(\overrightarrow{\mathrm{B})}, \mathrm{X}_{k} \in \overrightarrow{(\mathrm{A})}\right.$ and $\mathrm{Y}_{k} \in \overrightarrow{(\mathrm{B})}$. Thus, $\otimes_{k=1}^{s} \delta^{\mathrm{Y}_{k}}<\otimes_{k=1}^{n} \delta^{\mathrm{x}_{k}}$ with $\mathrm{X}_{k} \in \overrightarrow{(\mathrm{A})}$ and $\mathrm{Y}_{k} \in \overrightarrow{(\mathrm{B})}$. Hence, we have a contradiction since by hypothesis $\otimes_{k=1}^{n} \delta^{\mathrm{X}_{k}} \leq$ $\otimes_{k=1}^{s} \delta^{\mathrm{Y}_{k}}$ with $\mathrm{X}_{k} \in \overrightarrow{(\mathrm{A})}, 1 \leq k \leq n$, and $\mathrm{Y}_{k} \in \overrightarrow{(\mathrm{B})}, 1 \leq k \leq s$.

Directionality. Let $\left\langle\alpha^{\mathrm{A}}, \mu^{\mathrm{A}}, \delta^{\mathrm{A}}\right\rangle \in \lambda(\Theta)$ be a valuation associated with argument $\mathrm{A}$. Then:

(i) If there is no path in $\mathrm{G}_{\Theta}$ arriving to $\mathrm{A}$, then $\alpha^{\mathrm{A}}=\mu^{\mathrm{A}}=\delta^{\mathrm{A}}$.

(ii) If $\overrightarrow{(\mathrm{A})} \neq \emptyset$ and $\overrightarrow{(\mathrm{A})}=\emptyset$, then $\alpha^{\mathrm{A}} \leq \delta^{\mathrm{A}}$.

(iii) If $\overrightarrow{(\mathrm{A})}=\emptyset$ and $\overrightarrow{(\mathrm{A})} \neq \emptyset$, then $\alpha^{\mathrm{A}} \geq \delta^{\mathrm{A}}$.

(iv) If $\overrightarrow{(\mathrm{A})} \neq \emptyset$ and $\overrightarrow{(\mathrm{A})} \neq \emptyset$, then $\alpha^{\mathrm{A}} \leq \mu^{\mathrm{A}}$ and $\mu^{\mathrm{A}} \geq \delta^{\mathrm{A}}$.

We separate the proof in the corresponding four parts:

(i) If there is no path in $\mathrm{G}_{\Theta}$ arriving to $\mathrm{A}$, then $\overrightarrow{(\mathrm{A})}=\emptyset$ and $\overrightarrow{(\mathrm{A})}=\emptyset$. Thus, by Definition 11 , we have that $\alpha^{\mathrm{A}}=\rho(\mathrm{A}), \mu^{\mathrm{A}}=\alpha^{\mathrm{A}}$, and $\delta^{\mathrm{A}}=\mu^{\mathrm{A}}$. Then, the postulate is naturally proved based on the labeling process proposed in $L A F$.

(ii) If $\overrightarrow{(\mathrm{A})} \neq \emptyset$ and $\overrightarrow{(\mathrm{A})}=\emptyset$, then $\alpha^{\mathrm{A}} \leq \delta^{\mathrm{A}}$. By Lemma 2-(ii), we know that $\alpha^{\mathrm{A}} \leq \mu^{\mathrm{A}}$, while by Definition 11, we have that $\mu^{\mathrm{A}}=\delta^{\mathrm{A}}$ when $\overrightarrow{(\mathrm{A})}=\emptyset$. Finally, we can conclude that $\alpha^{\mathrm{A}} \leq \delta^{\mathrm{A}}$.

(iii) If $\overrightarrow{(\mathrm{A})}=\emptyset$ and $\overrightarrow{(\mathrm{A})} \neq \emptyset$, then $\alpha^{\mathrm{A}} \geq \delta^{\mathrm{A}}$. By Lemma 2-(i), we know that $\mu^{\mathrm{A}} \geq \delta^{\mathrm{A}}$, while by Definition 11, we have that $\mu^{\mathrm{A}}=\alpha^{\mathrm{A}}$. Finally, we can conclude that $\alpha^{\mathrm{A}} \geq \delta^{\mathrm{A}}$.

(iv) If $\overrightarrow{(\mathrm{A})} \neq \emptyset$ and $\overrightarrow{(\mathrm{A})} \neq \emptyset$, then $\alpha^{\mathrm{A}} \leq \mu^{\mathrm{A}}$ and $\mu^{\mathrm{A}} \geq \delta^{\mathrm{A}}$. By Definition 11, we know that $\mu^{\mathrm{A}}=\alpha^{\mathrm{A}} \oplus\left(\odot_{j=1}^{m} \delta^{\mathrm{x}_{j}}\right)$, with $\mathrm{X}_{j} \in(\overrightarrow{\mathrm{A}})$ when $(\mathrm{A}) \neq \emptyset$. Hence, $\alpha^{\mathrm{A}} \leq \mu^{\mathrm{A}}$. Furthermore, by Lemma 2-(i), we have that $\mu^{\mathrm{A}} \geq \delta^{\mathrm{A}}$.

EQUivalence. Let $\left\langle\alpha^{\mathrm{A}}, \mu^{\mathrm{A}}, \delta^{\mathrm{A}}\right\rangle,\left\langle\alpha^{\mathrm{B}}, \mu^{\mathrm{B}}, \delta^{\mathrm{B}}\right\rangle \in \lambda(\Theta)$ be the valuations associated with two arguments $\mathrm{A}$ and $\mathrm{B}$, respectively. Then, if it holds that

(i) $\alpha^{\mathrm{A}}=\alpha^{\mathrm{B}}$,

(ii) there exists a bijective function $f: \overrightarrow{(\mathrm{A})} \longrightarrow \overrightarrow{(\mathrm{B})}$ such that for all $\mathrm{x} \in \overrightarrow{(\mathrm{A})} \mu^{\mathrm{x}}=\mu^{f(\mathrm{x})}$,

(iii) there exists a bijective function $g: \overrightarrow{(\mathrm{A})} \longrightarrow \overrightarrow{(\mathrm{B})}$ such that for all $\mathrm{Y} \in \overrightarrow{(\mathrm{A})} \delta^{\mathrm{Y}}=\delta^{g(\mathrm{Y})}$, 
then $\left\langle\alpha^{\mathrm{A}}, \mu^{\mathrm{A}}, \delta^{\mathrm{A}}\right\rangle=\left\langle\alpha^{\mathrm{B}}, \mu^{\mathrm{B}}, \delta^{\mathrm{B}}\right\rangle$ saying that $\mathrm{A}$ and $\mathrm{B}$ are equivalent.

From the hypothesis $\alpha^{\mathrm{A}}=\alpha^{\mathrm{B}}, \delta^{\mathrm{A}_{j}}=\delta^{\mathrm{f}\left(\mathrm{A}_{\mathrm{j}}\right)}$ and $\delta^{\mathrm{X}_{k}}=\delta^{\mathrm{g}\left(\mathrm{X}_{\mathrm{k}}\right)}$, with $\mathrm{A}_{j} \in(\overrightarrow{\mathrm{A}})$ and $\mathrm{X}_{k} \in \overrightarrow{(\mathrm{A})}$. Furthermore $\odot_{k=1}^{n} \delta^{\mathrm{A}_{j}}=\odot_{k=1}^{n} \delta^{\mathrm{f}\left(\mathrm{A}_{\mathrm{j}}\right)}$ and $\otimes_{k=1}^{n} \delta^{\mathrm{x}_{k}}=\otimes_{k=1}^{n} \delta^{\mathrm{g}\left(\mathrm{x}_{\mathrm{k}}\right)}$ with $\mathrm{A}_{j} \in(\overrightarrow{\mathrm{A}})$ and $\mathrm{X}_{k} \in \overrightarrow{(\mathrm{A})}$. Hence for the above and Definition $11 \mu^{\mathrm{A}}=\mu^{\mathrm{B}}$ and $\delta^{\mathrm{A}}=\delta^{\mathrm{B}}$. Finally, we can conclude that $\left\langle\alpha^{\mathrm{A}}, \mu^{\mathrm{A}}, \delta^{\mathrm{A}}\right\rangle=\left\langle\alpha^{\mathrm{B}}, \mu^{\mathrm{B}}, \delta^{\mathrm{B}}\right\rangle$.

QuAlity Precedence. Given the valuations $\left\langle\alpha^{\mathrm{A}}, \mu^{\mathrm{A}}, \delta^{\mathrm{A}}\right\rangle,\left\langle\alpha^{\mathrm{B}}, \mu^{\mathrm{B}}, \delta^{\mathrm{B}}\right\rangle \in \lambda(\Theta)$ associated with two arguments $\mathrm{A}$ and $\mathrm{B}$, respectively. When $\alpha^{\mathrm{A}} \geq \alpha^{\mathrm{B}}$, we have that:

(i) If the strengths associated with the supporters of $\mathrm{A}$ and $\mathrm{B}$ verify that

$\odot_{j=1}^{m} \delta^{\mathrm{A}_{j}}>\odot_{j=1}^{r} \delta^{\mathrm{B}_{j}}$ with $\mathrm{A}_{j} \in(\overrightarrow{\mathrm{A}})$ and $\mathrm{B}_{j} \in(\overrightarrow{\mathrm{B}})$, then

$\delta^{\mathrm{A}}>\delta^{\mathrm{B}}$ when $\overrightarrow{(\mathrm{A})}=\emptyset$ and $\overrightarrow{(\mathrm{B})}=\emptyset$.

(ii) If the strengths associated with the attackers of $\mathrm{A}$ and $\mathrm{B}$ verify that

$\otimes_{k=1}^{n} \delta^{\mathrm{X}_{k}}<\otimes_{k=1}^{s} \delta^{\mathrm{Y}_{k}}$ with $\mathrm{X}_{k} \in \overrightarrow{(\mathrm{A})}$ and $\mathrm{Y}_{k} \in \overrightarrow{(\mathrm{B})}$, then

$\delta^{\mathrm{A}}>\delta^{\mathrm{B}}$ when $(\overrightarrow{\mathrm{A}})=\emptyset$ and $\overrightarrow{(\mathrm{B})}=\emptyset$.

We separate the proof in the corresponding two parts:

(i) By hypothesis we know that $\overrightarrow{(\mathrm{A})}=\emptyset$ and $\overrightarrow{(\mathrm{B})}=\emptyset$. Thus, by Definition 11, we have that $\delta^{\mathrm{A}}=\mu^{\mathrm{A}}$ and $\delta^{\mathrm{B}}=\mu^{\mathrm{B}}$. Furthermore, by Definition 11, we know that $\mu^{\mathrm{A}}=\alpha^{\mathrm{A}} \oplus\left(\odot_{j=1}^{m} \delta^{\mathrm{A}_{j}}\right)$ and $\mu^{\mathrm{B}}=\alpha^{\mathrm{B}} \oplus\left(\odot_{j=1}^{r} \delta^{\mathrm{B}_{j}}\right)$, with $\mathrm{A}_{j} \in(\overrightarrow{\mathrm{A}})$ and $\mathrm{B}_{j} \in(\overrightarrow{\mathrm{B}})$. Thus, $\delta^{\mathrm{A}}=\alpha^{\mathrm{A}} \oplus\left(\odot_{j=1}^{m} \delta^{\mathrm{A}_{j}}\right)$ and $\delta^{\mathrm{B}}=$ $\alpha^{\mathrm{B}} \oplus\left(\odot_{j=1}^{r} \delta^{\mathrm{B}_{j}}\right)$, with $\mathrm{A}_{j} \in(\overrightarrow{\mathrm{A}})$ and $\mathrm{B}_{j} \in \overrightarrow{(\mathrm{B})}$. By hypothesis, we know that $\alpha^{\mathrm{A}} \geq \alpha^{\mathrm{B}}$, and $\odot_{j=1}^{m} \delta^{\mathrm{A}_{j}}>\odot_{j=1}^{r} \delta^{\mathrm{B}_{j}}$ with $\mathrm{A}_{j} \in \overrightarrow{(\mathrm{A})}$ and $\mathrm{B}_{j} \in(\overrightarrow{\mathrm{B}})$. This leads us to conclude that $\delta^{\mathrm{A}}>\delta^{\mathrm{B}}$.

(ii) From the hypothesis we know that $\overrightarrow{(A)}=\emptyset$ and $(\vec{B})=\emptyset$. Thus, by Definition 11 , we have that $\delta^{\mathrm{A}}=\alpha^{\mathrm{A}} \ominus\left(\otimes_{k=1}^{n} \delta^{\mathrm{x}_{k}}\right)$ and $\delta^{\mathrm{B}}=\alpha^{\mathrm{B}} \ominus\left(\otimes_{k=1}^{s} \delta^{\mathrm{Y}_{k}}\right)$ with $\mathrm{X}_{k} \in \overrightarrow{(\mathrm{A})}$ and $\mathrm{Y}_{k} \in \overrightarrow{(\mathrm{A})}$. Furthermore, by hypothesis, we know that $\otimes_{k=1}^{n} \delta^{\mathrm{X}_{k}}<\otimes_{k=1}^{s} \delta^{\mathrm{Y}_{k}}$ and $\alpha^{\mathrm{A}} \geq \alpha^{\mathrm{B}}$. This leads us to conclude that $\delta^{\mathrm{A}}>\delta^{\mathrm{B}}$.

NeUtRALity. For any argument $\mathrm{A} \in \mathrm{AR}$ where $\left\langle\alpha^{\mathrm{A}}, \mu^{\mathrm{A}}, \delta^{\mathrm{A}}\right\rangle \in \lambda(\Theta)$ we have that: if $\odot_{j=1}^{m} \delta^{\mathrm{A}_{j}}=$ $\otimes_{k=1}^{n} \delta^{\mathrm{x}_{k}}=\perp$ with $\mathrm{A}_{j} \in \overrightarrow{(\mathrm{A})}, 1 \leq j \leq m$, and $\mathrm{x}_{k} \in \overrightarrow{(\mathrm{A})}, 1 \leq k \leq n$, then $\delta^{\mathrm{A}}=\alpha^{\mathrm{A}}$.

From the hypothesis, we know that $\odot_{j=1}^{m} \delta^{\mathrm{A}_{j}}=\otimes_{k=1}^{n} \delta^{\mathrm{x}_{k}}=\perp$ with $\mathrm{X}_{k} \in \overrightarrow{(\mathrm{A})}$ and $\mathrm{A}_{j} \in \overrightarrow{(\mathrm{A})}$. Furthermore, by Definition 11, we have that $\delta^{\mathrm{A}}=\alpha^{\mathrm{A}} \oplus\left(\odot_{j=1}^{m} \delta^{\mathrm{A}_{j}} \ominus \otimes_{k=1}^{n} \delta^{\mathrm{X}_{k}}\right)$ and $\mu^{\mathrm{A}}=\alpha^{\mathrm{A}} \oplus\left(\odot_{j=1}^{n} \delta^{\mathrm{A}_{j}}\right)$ with $\mathrm{X}_{k} \in \overrightarrow{(\mathrm{A})}$ and $\mathrm{A}_{j} \in \overrightarrow{(\mathrm{A})}$. Hence, we can infer that $\delta^{\mathrm{A}}=\alpha^{\mathrm{A}} \oplus \perp$ and $\mu^{\mathrm{A}}=\alpha^{\mathrm{A}} \oplus \perp$. In addition, by Definition 8, we know that $\perp$ is the neutral element for $\ominus$ and $\oplus$. Thus, we can deduce that $\delta^{\mathrm{A}}=\mu^{\mathrm{A}}=\alpha^{\mathrm{A}}$. Then, $\delta^{\mathrm{A}}=\alpha^{\mathrm{A}}$.

REINFORCEMENT. For any argument $\mathrm{A} \in \mathrm{AR}$ and $\left\langle\alpha^{\mathrm{A}}, \mu^{\mathrm{A}}, \delta^{\mathrm{A}}\right\rangle \in \lambda(\Theta)$ then $\delta^{\mathrm{A}}>\alpha^{\mathrm{A}}$ iff $\odot_{j=1}^{m} \delta^{\mathrm{A}_{j}}>$ $\otimes_{k=1}^{n} \delta^{\mathrm{x}_{k}}$, where $\mathrm{A}_{j} \in \overrightarrow{(\mathrm{A})}, 1 \leq j \leq m$ and $\mathrm{X}_{k} \in \overrightarrow{(\mathrm{A})}, 1 \leq k \leq n$. 
From the hypothesis, we know that $\odot_{j=1}^{m} \delta^{\mathrm{A}_{j}}>\otimes_{k=1}^{n} \delta^{\mathrm{x}_{k}}$ with $\mathrm{X}_{k} \in \overrightarrow{(\mathrm{A})}$ and $\mathrm{A}_{j} \in \overrightarrow{(\mathrm{A})}$. Thus, $\odot_{j=1}^{m} \delta^{\mathrm{A}_{j}} \ominus$ $\otimes_{k=1}^{n} \delta^{\mathrm{x}_{k}}>\perp$ with $\mathrm{X}_{k} \in \overrightarrow{(\mathrm{A})}$ and $\mathrm{A}_{j} \in(\overrightarrow{\mathrm{A}})$. Since by Definition $11, \delta^{\mathrm{A}}=\alpha^{\mathrm{A}} \oplus\left(\odot_{j=1}^{m} \delta^{\mathrm{A}_{j}} \ominus \otimes_{k=1}^{n} \delta^{\mathrm{X}_{k}}\right)$ with $\mathrm{A}_{j} \in(\overrightarrow{\mathrm{A}})$ and $\mathrm{X}_{k} \in \overrightarrow{(\mathrm{A})}$, we can deduce that $\delta^{\mathrm{A}}>\alpha^{\mathrm{A}}$.

STABILITy. For any argument A where $\left\langle\alpha^{\mathrm{A}}, \mu^{\mathrm{A}}, \delta^{\mathrm{A}}\right\rangle \in \lambda(\Theta)$ if $\overrightarrow{(\mathrm{A})}=\emptyset$ and $\overrightarrow{(\mathrm{A})}=\emptyset$ we have that $\delta^{\mathrm{A}}=\alpha^{\mathrm{A}}$.

From the hypothesis we have that $\overrightarrow{(\mathrm{A})}=\emptyset$ and $\overrightarrow{(\mathrm{A})}=\emptyset$. Hence, by Definition 11 , we know that $\mu^{\mathrm{A}}=\alpha^{\mathrm{A}}$ and $\delta^{\mathrm{A}}=\mu^{\mathrm{A}}$. Thus, we can deduce that $\delta^{\mathrm{A}}=\alpha^{\mathrm{A}}$.

WEAKENING. For any argument A where $\left\langle\alpha^{\mathrm{A}}, \mu^{\mathrm{A}}, \delta^{\mathrm{A}}\right\rangle \in \lambda(\Theta)$, we have that:

(i) If $\otimes_{k=1}^{n} \delta^{\mathrm{x}_{k}}>\odot_{j=1}^{m} \delta^{\mathrm{A}_{j}}$ with $\mathrm{X}_{k} \in \overrightarrow{(\mathrm{A})}, 1 \leq k \leq n$, and $\mathrm{A}_{j} \in \overrightarrow{(\mathrm{A})}, 1 \leq j \leq m$, then $\alpha^{\mathrm{A}}>\delta^{\mathrm{A}}$.

(ii) If $\overrightarrow{(\mathrm{A})} \neq \emptyset$ and $\overrightarrow{(\mathrm{A})}=\emptyset$, then $\alpha^{\mathrm{A}} \geq \delta^{\mathrm{A}}$.

We separate the proof in two parts:

(i) if $\otimes_{k=1}^{n} \delta^{\mathrm{x}_{k}}>\odot_{j=1}^{m} \delta^{\mathrm{A}_{j}}$ with $\mathrm{X}_{k} \in \overrightarrow{(\mathrm{A})}$ and $\mathrm{A}_{j} \in \overrightarrow{(\mathrm{A})}$, then $\alpha^{\mathrm{A}} \geq \delta^{\mathrm{A}}$. By Definition 11, if $\otimes_{k=1}^{n} \delta^{\mathrm{x}_{k}}>$ $\odot_{j=1}^{m} \delta^{\mathrm{A}_{j}}$ with $\mathrm{X}_{k} \in \overrightarrow{(\mathrm{A})}$ and $\mathrm{A}_{j} \in \overrightarrow{(\mathrm{A})}$, then $\delta^{\mathrm{A}}=\alpha^{\mathrm{A}} \ominus\left(\otimes_{k=1}^{n} \delta^{\mathrm{X}_{k}} \ominus \odot_{j=1}^{m} \delta^{\mathrm{A}_{j}}\right)$. Furthermore, $\otimes_{k=1}^{n} \delta^{\mathrm{x}_{k}} \ominus \odot_{j=1}^{m} \delta^{\mathrm{A}_{j}}>\perp$. This leads us to conclude that $\alpha^{\mathrm{A}}>\delta^{\mathrm{A}}$.

(ii) if $\overrightarrow{(\mathrm{A})} \neq \emptyset$ and $\overrightarrow{(\mathrm{A})}=\emptyset$, then $\alpha^{\mathrm{A}}>\delta^{\mathrm{A}}$. By Definition 11 , if $\overrightarrow{(\mathrm{A})} \neq \emptyset$ and $\overrightarrow{(\mathrm{A})}=\emptyset$, then $\delta^{\mathrm{A}}=$ $\alpha^{\mathrm{A}} \ominus\left(\otimes_{k=1}^{n} \delta^{\mathrm{x}_{k}}\right)$ with $\mathrm{X}_{k} \in \overrightarrow{(\mathrm{A})}$. Furthermore, $\otimes_{k=1}^{n} \delta^{\mathrm{x}_{k}} \geq \perp$. This leads us to conclude that $\alpha^{\mathrm{A}} \geq \delta^{\mathrm{A}}$.

StREnGthening. For any argument A where $\left\langle\alpha^{\mathrm{A}}, \mu^{\mathrm{A}}, \delta^{\mathrm{A}}\right\rangle \in \lambda(\Theta)$, we have that:

(i) If $\otimes_{k=1}^{n} \delta^{\mathrm{x}_{k}} \leq \odot_{j=1}^{m} \delta^{\mathrm{A}_{j}}$ with $\mathrm{X}_{k} \in \overrightarrow{(\mathrm{A})}, 1 \leq k \leq n$, and $\mathrm{A}_{j} \in \overrightarrow{(\mathrm{A})}, 1 \leq j \leq m$, then $\alpha^{\mathrm{A}} \leq \delta^{\mathrm{A}}$.

(ii) If $\overrightarrow{(\mathrm{A})}=\emptyset$, then $\alpha^{\mathrm{A}} \leq \delta^{\mathrm{A}}$.

We separate the proof in two parts:

(i) if $\otimes_{k=1}^{n} \delta^{\mathrm{x}_{k}} \leq \odot_{j=1}^{m} \delta^{\mathrm{A}_{j}}$ with $\mathrm{X}_{k} \in \overrightarrow{(\mathrm{A})}$ and $\mathrm{A}_{j} \in \overrightarrow{(\mathrm{A})}$, then $\alpha^{\mathrm{A}} \leq \delta^{\mathrm{A}}$. By Definition 11, if $\otimes_{k=1}^{n} \delta^{\mathrm{x}_{k}} \leq$ $\odot_{j=1}^{m} \delta^{\mathrm{A}_{j}}$ with $\mathrm{X}_{k} \in \overrightarrow{(\mathrm{A})}$ and $\mathrm{A}_{j} \in \overrightarrow{(\mathrm{A})}$, then $\delta^{\mathrm{A}}=\alpha^{\mathrm{A}} \oplus\left(\odot_{j=1}^{m} \delta^{\mathrm{A}_{j}} \ominus \otimes_{k=1}^{n} \delta^{\mathrm{X}_{k}}\right)$. Furthermore, $\odot_{j=1}^{m} \delta^{\mathrm{A}_{j}} \ominus \otimes_{k=1}^{n} \delta^{\mathrm{x}_{k}} \geq \perp$. This leads us to conclude that $\alpha^{\mathrm{A}} \leq \delta^{\mathrm{A}}$.

(ii) if $\overrightarrow{(\mathrm{A})}=\emptyset$, then $\alpha^{\mathrm{A}} \leq \delta^{\mathrm{A}}$. By Definition 11 , if $\overrightarrow{(\mathrm{A})}=\emptyset$, then $\delta^{\mathrm{A}}=\mu^{\mathrm{A}}$. Thus, by Lemma 2- $(i)$, we know that $\mu^{\mathrm{A}} \geq \alpha^{\mathrm{A}}$. This leads us to conclude that $\alpha^{\mathrm{A}} \leq \delta^{\mathrm{A}}$.

CAUSAlity. For any argument $\mathrm{A}$ where $\left\langle\alpha^{\mathrm{A}}, \mu^{\mathrm{A}}, \delta^{\mathrm{A}}\right\rangle \in \lambda(\Theta)$. Then:

(i) If $\mu^{\mathrm{A}} \neq \delta^{\mathrm{A}}$ there exists an argument $\mathrm{B} \in \mathrm{AR}$ such that $\delta^{\mathrm{B}} \neq \perp$ with $\mathrm{B} \in \overrightarrow{(\mathrm{A})}$.

(ii) If $\alpha^{\mathrm{A}} \neq \mu^{\mathrm{A}}$ there exists an argument $\mathrm{B} \in \mathrm{AR}$ such that $\delta^{\mathrm{B}} \neq \perp$ with $\mathrm{B} \in \overrightarrow{(\mathrm{A})}$. 
We separate the proof in two parts:

(i) If $\mu^{\mathrm{A}} \neq \delta^{\mathrm{A}}$ there exists an argument $\mathrm{X}_{k} \in \mathrm{Arg}$ such that $\delta^{\mathrm{x}_{k}} \neq \perp$ with $\mathrm{X} \in \overrightarrow{(\mathrm{A})}$. By hypothesis $\mu^{\mathrm{A}} \neq \delta^{\mathrm{A}}$, then $\overrightarrow{(\mathrm{A})} \neq \emptyset$.

- If $(\overrightarrow{\mathrm{A}})=\emptyset$ then by Definition 11 we know that $\mu^{\mathrm{A}}=\alpha^{\mathrm{A}}$ and $\delta^{\mathrm{A}}=\alpha^{\mathrm{A}} \ominus\left(\otimes_{k=1}^{n} \delta^{\mathrm{x}_{k}}\right)$ with $\mathrm{X}_{k} \in \overrightarrow{(\mathrm{A})}$. Since $\mu^{\mathrm{A}} \neq \delta^{\mathrm{A}}$, then $\otimes_{k=1}^{n} \delta^{\mathrm{x}_{k}}>\perp$. This leads us to conclude that there exists $\mathrm{X}_{k} \in \overrightarrow{(\mathrm{A})}$ such that $\delta^{\mathrm{x}_{k}} \neq \perp$.

- If $\overrightarrow{(\mathrm{A})} \neq \emptyset$ then by Definition 11 we know that $\mu^{\mathrm{A}}=\alpha^{\mathrm{A}} \oplus\left(\odot_{j=1}^{m} \delta^{\mathrm{A}_{j}}\right)$, with $\mathrm{A}_{j} \in(\overrightarrow{\mathrm{A}})$. Furthermore, the overall valuation is obtained by the following cases: If $\otimes_{k=1}^{n} \delta^{\mathrm{x}_{k}} \leq \odot_{j=1}^{m} \delta^{\mathrm{A}_{j}}$ then $\delta^{\mathrm{A}}=\alpha^{\mathrm{A}} \oplus\left(\odot_{j=1}^{m} \delta^{\mathrm{A}_{j}} \ominus \otimes_{k=1}^{n} \delta^{\mathrm{x}_{k}}\right)$ with $\mathrm{X}_{k} \in \overrightarrow{(\mathrm{A})}$ and $\mathrm{A}_{j} \in \overrightarrow{(\mathrm{A})}$. Since $\mu^{\mathrm{A}} \neq \delta^{\mathrm{A}}$, then $\odot_{j=1}^{m} \delta^{\mathrm{A}_{j}}>\odot_{j=1}^{m} \delta^{\mathrm{A}_{j}} \ominus \otimes_{k=1}^{n} \delta^{\mathrm{X}_{k}}$. This leads us to conclude that there exists $\mathrm{X}_{k} \in \overrightarrow{(\mathrm{A})}$ such that $\delta^{\mathrm{X}_{k}} \neq \perp$.

If $\otimes_{k=1}^{n} \delta^{\mathrm{X}_{k}}>\odot_{j=1}^{m} \delta^{\mathrm{A}_{j}}$ then $\delta^{\mathrm{A}}=\alpha^{\mathrm{A}} \ominus\left(\otimes_{k=1}^{n} \delta^{\mathrm{X}_{k}} \ominus \odot_{j=1}^{m} \delta^{\mathrm{A}_{j}}\right)$ with $\mathrm{X}_{k} \in \overrightarrow{(\mathrm{A})}$ and $\mathrm{A}_{j} \in(\overrightarrow{\mathrm{A}})$. Since $\otimes_{k=1}^{n} \delta^{\mathrm{x}_{k}}>\odot_{j=1}^{m} \delta^{\mathrm{A}_{j}}$ we can deduce that $\otimes_{k=1}^{n} \delta^{\mathrm{x}_{k}}>\perp$. This leads us to conclude that there exists $\mathrm{X}_{k} \in \overrightarrow{(\mathrm{A})}$ such that $\delta^{\mathrm{x}_{k}} \neq \perp$.

(ii) If $\alpha^{\mathrm{A}} \neq \mu^{\mathrm{A}}$ there exists an argument $\mathrm{A}_{j} \in \mathrm{Arg}$ such that $\delta^{\mathrm{A}_{j}} \neq \perp$ with $\mathrm{A}_{j} \in(\overrightarrow{\mathrm{A}})$. If $\alpha^{\mathrm{A}} \neq \mu^{\mathrm{A}}$, then by Definition 11, we know that $\mu^{\mathrm{A}}=\alpha^{\mathrm{A}} \oplus\left(\odot_{j=1}^{m} \delta^{\mathrm{A}_{j}}\right)$, with $\mathrm{A}_{j} \in \overrightarrow{(\mathrm{A})}$. Since $\alpha^{\mathrm{A}} \neq \mu^{\mathrm{A}}$, then $\odot_{j=1}^{m} \delta^{\mathrm{A}_{j}}>\perp$. This leads us to conclude that there exists $\mathrm{A}_{j} \in \overrightarrow{(\mathrm{A})}$ such that $\delta^{\mathrm{A}_{j}} \neq \perp$.

NeUtrality. For any argument $\mathrm{A} \in \mathrm{AR}$ where $\left\langle\alpha^{\mathrm{A}}, \mu^{\mathrm{A}}, \delta^{\mathrm{A}}\right\rangle \in \lambda(\Theta)$, if it holds that $\odot_{j=1}^{m} \delta^{\mathrm{A}_{j}}=$ $\otimes_{k=1}^{n} \delta^{\mathrm{x}_{k}}$ with $\mathrm{A}_{j} \in \overrightarrow{(\mathrm{A})}, 1 \leq j \leq m$, and $\mathrm{X}_{k} \in \overrightarrow{(\mathrm{A})}, 1 \leq k \leq n$, then $\alpha^{\mathrm{A}}=\delta^{\mathrm{A}}$.

By Definition 11, we know that $\delta^{\mathrm{A}}=\alpha^{\mathrm{A}} \oplus\left(\odot_{j=1}^{m} \delta^{\mathrm{A}_{j}} \ominus \otimes_{k=1}^{n} \delta^{\mathrm{x}_{k}}\right)$, with $\mathrm{X}_{k} \in \overrightarrow{(\mathrm{A})}$ and $\mathrm{A}_{j} \in \overrightarrow{(\mathrm{A})}$. Since $\odot_{j=1}^{m} \delta^{\mathrm{A}_{j}}=\otimes_{k=1}^{n} \delta^{\mathrm{X}_{k}}$, then we can conclude that $\delta^{\mathrm{A}}=\alpha^{\mathrm{A}} \oplus \perp$. Finally, $\alpha^{\mathrm{A}}=\delta^{\mathrm{A}}$.

Proportionality. For any arguments $\mathrm{A}, \mathrm{B}$ where $\left\langle\alpha^{\mathrm{A}}, \mu^{\mathrm{A}}, \delta^{\mathrm{A}}\right\rangle,\left\langle\alpha^{\mathrm{B}}, \mu^{\mathrm{B}}, \delta^{\mathrm{B}}\right\rangle \in \lambda(\Theta)$, if

(i) $\alpha^{\mathrm{A}}>\alpha^{\mathrm{B}}$,

(ii) $\stackrel{\overrightarrow{\mathrm{B}})}{\overrightarrow{\mathrm{B}}}=\emptyset$ or $\odot_{j=1}^{m} \delta^{\mathrm{A}_{j}} \geq \odot_{k=1}^{r} \delta^{\mathrm{B}_{\mathrm{k}}}$ with $\mathrm{A}_{j} \in \overrightarrow{(\mathrm{A})}, 1 \leq j \leq m$, and $\mathrm{B}_{k} \in \overrightarrow{(\mathrm{B})}, 1 \leq k \leq n$,

(iii) $\otimes_{j=1}^{m} \delta^{\mathrm{x}_{j}}=\otimes_{k=1}^{n} \delta^{\mathrm{Y}_{\mathrm{k}}}$ with $\mathrm{X}_{j} \in \overrightarrow{(\mathrm{A})}, 1 \leq j \leq m$, and $\mathrm{Y}_{k} \in \overrightarrow{(\mathrm{B})}, 1 \leq k \leq n$, and

(iv) $\delta^{\mathrm{A}}>\perp$.

then $\delta^{\mathrm{A}}>\delta^{\mathrm{B}}$.

We separate the proof in three parts:

- If $\overrightarrow{(\mathrm{A})}=\overrightarrow{(\mathrm{B})}=\emptyset$, then by Definition 11 we know that $\delta^{\mathrm{A}}=\alpha^{\mathrm{A}} \ominus\left(\otimes_{k=1}^{n} \delta^{\mathrm{x}_{k}}\right)$ with $\mathrm{X}_{k} \in \overrightarrow{(\mathrm{A})}$ and $\delta^{\mathrm{B}}=\alpha^{\mathrm{B}} \ominus\left(\otimes_{k=1}^{s} \delta^{\mathrm{Y}_{k}}\right)$ with $\mathrm{Y}_{k} \in \overrightarrow{(\mathrm{B})}$. By Hypothesis we have that $\otimes_{k=1}^{n} \delta^{\mathrm{X}_{k}}=\otimes_{k=1}^{s} \delta^{\mathrm{Y}_{k}}$ with $\mathrm{X}_{k} \in \overrightarrow{(\mathrm{A})}$ and $\mathrm{Y}_{k} \in \overrightarrow{(\mathrm{B})}$, and $\alpha^{\mathrm{A}}>\alpha^{\mathrm{B}}$. Thus, we can deduce that $\delta^{\mathrm{A}}>\delta^{\mathrm{B}}$. 
- If $\overrightarrow{(\mathrm{A})} \neq \emptyset$ and $\overrightarrow{(\mathrm{B})}=\emptyset$, then by Definition 11 we know that $\delta^{\mathrm{B}}=\alpha^{\mathrm{B}} \ominus\left(\otimes_{k=1}^{s} \delta^{\mathrm{Y}_{k}}\right)$ with $\mathrm{Y}_{k} \in \overrightarrow{(\mathrm{B})}$, and we analyze the following cases:

- If $\otimes_{k=1}^{n} \delta^{\mathrm{x}_{k}} \leq \odot_{j=1}^{m} \delta^{\mathrm{A}_{j}}$ with $\mathrm{X}_{k} \in \overrightarrow{(\mathrm{A})}$ and $\mathrm{A}_{j} \in \overrightarrow{(\mathrm{A})}$, then by Definition 11 we know that $\delta^{\mathrm{A}}=\alpha^{\mathrm{A}} \oplus\left(\odot_{j=1}^{m} \delta^{\mathrm{A}_{j}} \ominus \otimes_{k=1}^{n} \delta^{\mathrm{x}_{k}}\right)$, with $\mathrm{X}_{k} \in \overrightarrow{(\mathrm{A})}$ and $\mathrm{A}_{j} \in \overrightarrow{(\mathrm{A})}$. By hypothesis we have that $\alpha^{\mathrm{A}}>\alpha^{\mathrm{B}}$, then we can deduce that $\delta^{\mathrm{A}}>\delta^{\mathrm{B}}$.

- If $\otimes_{k=1}^{n} \delta^{\mathrm{x}_{k}}>\odot_{j=1}^{m} \delta^{\mathrm{A}_{j}}$ with $\mathrm{X}_{k} \in \overrightarrow{(\mathrm{A})}$ and $\mathrm{A}_{j} \in(\overrightarrow{\mathrm{A}})$, then by Definition 11 we know that $\delta^{\mathrm{A}}=\alpha^{\mathrm{A}} \ominus\left(\otimes_{k=1}^{n} \delta^{\mathrm{x}_{k}} \ominus \odot_{j=1}^{m} \delta^{\mathrm{A}_{j}}\right)$ with $\mathrm{X}_{k} \in \overrightarrow{(\mathrm{A})}$ and $\mathrm{A}_{j} \in \overrightarrow{(\mathrm{A})}$. By hypothesis we have that $\otimes_{k=1}^{n} \delta^{\mathrm{x}_{k}}=\otimes_{k=1}^{s} \delta^{\mathrm{Y}_{k}}$ with $\mathrm{X}_{k} \in \overrightarrow{(\mathrm{A})}$ and $\mathrm{Y}_{k} \in \overrightarrow{(\mathrm{B})}$. Thus, we can deduce that $\otimes_{k=1}^{n} \delta^{\mathrm{X}_{k}} \ominus$ $\odot_{j=1}^{m} \delta^{\mathrm{A}_{j}}<\otimes_{k=1}^{s} \delta^{\mathrm{Y}_{k}}$ with $\mathrm{A}_{j} \in \overrightarrow{(\mathrm{A})}, \mathrm{X}_{k} \in \overrightarrow{(\mathrm{A})}$ and $\mathrm{Y}_{k} \in \overrightarrow{(\mathrm{B})}$. Furthermore, by hypothesis we know that $\alpha^{\mathrm{A}}>\alpha^{\mathrm{B}}$, then we can deduce that $\delta^{\mathrm{A}}>\delta^{\mathrm{B}}$.

- If $\overrightarrow{(\mathrm{A})} \neq \emptyset$ and $\overrightarrow{(\mathrm{B})} \neq \emptyset$, then by Definition 11 we know that $\mu^{\mathrm{A}}=\alpha^{\mathrm{A}} \oplus\left(\odot_{j=1}^{m} \delta^{\mathrm{A}_{j}}\right)$ and $\mu^{\mathrm{B}}=$ $\alpha^{\mathrm{B}} \oplus\left(\odot_{j=1}^{r} \delta^{\mathrm{B}_{j}}\right)$, with $\mathrm{A}_{j} \in \overrightarrow{(\mathrm{A})}$ and $\mathrm{B}_{j} \in(\overrightarrow{\mathrm{B}})$.

- If $\otimes_{k=1}^{n} \delta^{\mathrm{X}_{k}} \leq \odot_{j=1}^{m} \delta^{\mathrm{A}_{j}}$ with $\mathrm{X}_{k} \in \overrightarrow{(\mathrm{A})}$ and $\mathrm{A}_{j} \in \overrightarrow{(\mathrm{A})}$, then by Definition 11 we know that $\delta^{\mathrm{A}}=\alpha^{\mathrm{A}} \oplus\left(\odot_{j=1}^{m} \delta^{\mathrm{A}_{j}} \ominus \otimes_{k=1}^{n} \delta^{\mathrm{x}_{k}}\right)$, with $\mathrm{X}_{k} \in \overrightarrow{(\mathrm{A})}$ and $\mathrm{A}_{j} \in \overrightarrow{(\mathrm{A})}$. Then, we analyze the following cases:

- If $\otimes_{k=1}^{s} \delta^{\mathrm{Y}_{k}} \leq \odot_{j=1}^{r} \delta^{\mathrm{B}_{j}}$ with $\mathrm{Y}_{k} \in \overrightarrow{(\mathrm{B})}$ and $\mathrm{B}_{j} \in \overrightarrow{(\mathrm{B})}$, then by Definition 11 we know that $\delta^{\mathrm{B}}=\alpha^{\mathrm{B}} \oplus\left(\odot_{j=1}^{r} \delta^{\mathrm{B}_{j}} \ominus \otimes_{k=1}^{s} \delta^{\mathrm{Y}_{k}}\right)$, with $\mathrm{Y}_{k} \in \overrightarrow{(\mathrm{B})}$ and $\mathrm{B}_{j} \in(\overrightarrow{\mathrm{B}})$. By hypothesis we know that $\alpha^{\mathrm{A}}>\alpha^{\mathrm{B}}, \odot_{j=1}^{m} \delta^{\mathrm{A}_{j}} \geq \odot_{j=1}^{r} \delta^{\mathrm{B}_{j}}$ with $\mathrm{A}_{j} \in(\overrightarrow{\mathrm{A}})$ and $\mathrm{B}_{j} \in(\overrightarrow{\mathrm{B}})$, and $\otimes_{k=1}^{n} \delta^{\mathrm{x}_{k}}=$ $\otimes_{k=1}^{s} \delta^{\mathrm{Y}_{k}}$ with $\mathrm{X}_{k} \in \overrightarrow{(\mathrm{A})}$ and $\mathrm{Y}_{k} \in \overrightarrow{(\mathrm{B})}$. Thus, we can deduce that $\delta^{\mathrm{A}}>\delta^{\mathrm{B}}$.

- If $\otimes_{k=1}^{s} \delta^{\mathrm{Y}_{k}}>\odot_{j=1}^{r} \delta^{\mathrm{B}_{j}}$ with $\mathrm{Y}_{k} \in \overrightarrow{(\mathrm{B})}$ and $\mathrm{B}_{j} \in \overrightarrow{(\mathrm{B})}$, then by Definition 11 we know that $\delta^{\mathrm{B}}=\alpha^{\mathrm{B}} \ominus\left(\otimes_{k=1}^{s} \delta^{\mathrm{Y}_{k}} \ominus \odot_{j=1}^{r} \delta^{\mathrm{B}_{j}}\right)$ with $\mathrm{Y}_{k} \in \overrightarrow{(\mathrm{B})}$ and $\mathrm{B}_{j} \in(\overrightarrow{\mathrm{B}})$. Then, we can deduce that $\delta^{\mathrm{A}}>\delta^{\mathrm{B}}$.

- If $\otimes_{k=1}^{n} \delta^{\mathrm{X}_{k}}>\odot_{j=1}^{m} \delta^{\mathrm{A}_{j}}$ with $\mathrm{X}_{k} \in \overrightarrow{(\mathrm{A})}$ and $\mathrm{A}_{j} \in(\overrightarrow{\mathrm{A}})$, then by hypothesis we have that $\odot_{j=1}^{m} \delta^{\mathrm{A}_{j}} \geq \odot_{j=1}^{r} \delta^{\mathrm{B}_{j}}$ with $\mathrm{A}_{j} \in \overrightarrow{(\mathrm{A})}$ and $\mathrm{B}_{j} \in \overrightarrow{(\mathrm{B})}$, and $\otimes_{k=1}^{n} \delta^{\mathrm{X}_{k}}=\otimes_{k=1}^{s} \delta^{\mathrm{Y}_{k}}$ with $\mathrm{X}_{k} \in \overrightarrow{(\mathrm{A})}$ and $\mathrm{Y}_{k} \in \overrightarrow{(\mathrm{B})}$. Furthermore by Definition 11 we know that $\alpha^{\mathrm{A}} \ominus\left(\otimes_{k=1}^{n} \delta^{\mathrm{X}_{k}} \ominus \odot_{j=1}^{m} \delta^{\mathrm{A}_{j}}\right)$ with $\mathrm{X}_{k} \in \overrightarrow{(\mathrm{A})}$ and $\mathrm{A}_{j} \in \overrightarrow{(\mathrm{A})}$. Then, we analyze the following cases:

- If $\otimes_{k=1}^{s} \delta^{\mathrm{Y}_{k}} \leq \odot_{j=1}^{r} \delta^{\mathrm{B}_{j}}$ with $\mathrm{Y}_{k} \in \overrightarrow{(\mathrm{B})}$ and $\mathrm{B}_{j} \in \overrightarrow{(\mathrm{B})}$, we have a contradiction. Since $\otimes_{k=1}^{n} \delta^{\mathrm{X}_{k}}>\odot_{j=1}^{m} \delta^{\mathrm{A}_{j}}$ with $\mathrm{X}_{k} \in \overrightarrow{(\mathrm{A})}$ and $\mathrm{A}_{j} \in \overrightarrow{(\mathrm{A})}$, then $\odot_{k=1}^{m} \delta^{\mathrm{A}_{j}}<\odot_{j=1}^{r} \delta^{\mathrm{B}_{j}}$ with $\mathrm{A}_{j} \in(\overrightarrow{\mathrm{A}})$ and $\mathrm{B}_{j} \in(\overrightarrow{\mathrm{B}})$. 
- If $\otimes_{k=1}^{s} \delta^{\mathrm{Y}_{k}}>\odot_{j=1}^{r} \delta^{\mathrm{B}_{j}}$ with $\mathrm{Y}_{k} \in \overrightarrow{(\mathrm{B})}$ and $\mathrm{B}_{j} \in(\overrightarrow{\mathrm{B})}$, by Definition 11 we know that $\delta^{\mathrm{B}}=\alpha^{\mathrm{B}} \ominus\left(\otimes_{k=1}^{s} \delta^{\mathrm{Y}_{k}} \ominus \odot_{j=1}^{r} \delta^{\mathrm{B}_{j}}\right)$ with $\mathrm{Y}_{k} \in \overrightarrow{(\mathrm{B})}$ and $\mathrm{B}_{j} \in(\overrightarrow{\mathrm{B}})$. Hence, we can deduce that $\otimes_{k=1}^{n} \delta^{\mathrm{X}_{k}} \ominus \odot_{j=1}^{m} \delta^{\mathrm{A}_{j}} \leq \otimes_{k=1}^{s} \delta^{\mathrm{Y}_{k}} \ominus \odot_{j=1}^{r} \delta^{\mathrm{B}_{j}}$. Furthermore, by hypothesis we know that $\alpha^{\mathrm{A}}>\alpha^{\mathrm{B}}$. Thus, we conclude that $\delta^{\mathrm{A}}>\delta^{\mathrm{B}}$.

\section{References}

Alsinet, T., Chesñevar, C. I., Godo, L., \& Simari, G. R. (2008a). A logic programming framework for possibilistic argumentation: Formalization and logical properties. Fuzzy Sets and Systems, 159(10), 1208-1228.

Alsinet, T., Chesñevar, C. I., Godo, L., Sandri, S. A., \& Simari, G. R. (2008b). Formalizing argumentative reasoning in a possibilistic logic programming setting with fuzzy unification. Int. J. Approx. Reasoning, 48(3), 711-729.

Amgoud, L., Cayrol, C., Lagasquie-Schiex, M. C., \& Livet, P. (2008). On bipolarity in argumentation frameworks. Intelligent Systems, 23(10), 1062-1093.

Amgoud, L., \& Ben-Naim, J. (2013). Ranking-based semantics for argumentation frameworks. In International Conference on Scalable Uncertainty Management, pp. 134-147. Springer.

Amgoud, L., \& Ben-Naim, J. (2016). Evaluation of arguments from support relations: Axioms and semantics. In Kambhampati, S. (Ed.), Proc. of the Twenty-Fifth International Joint Conference on Artificial Intelligence, IJCAI New York, USA, pp. 900-906. IJCAI/AAAI Press.

Amgoud, L., \& Ben-Naim, J. (2018). Weighted bipolar argumentation graphs: Axioms and semantics. In Lang, J. (Ed.), Proceedings of the Twenty-Seventh International Joint Conference on Artificial Intelligence, IJCAI, pp. 5194-5198, Stockholm, Sweden. ijcai.org.

Amgoud, L., Ben-Naim, J., \& Vesic, S. (2017). Measuring the intensity of attacks in argumentation graphs with shapley value..

Amgoud, L., \& Cayrol, C. (1998). On the acceptability of arguments in preference-based argumentation. In Proceedings of the Fourteenth conference on Uncertainty in artificial intelligence, pp. 1-7. Morgan Kaufmann Publishers Inc.

Amgoud, L., Cayrol, C., \& Lagasquie-Schiex, M. C. (2004). On the bipolarity in argumentation frameworks. In Delgrande, J., \& Schaub, T. (Eds.), 10th International Workshop on NonMonotonic Reasoning (NMR 2004), Whistler, Canada, June 6-8, 2004, Proceedings, pp. 1-9.

Amgoud, L., \& Doder, D. (2019). Gradual semantics accounting for varied-strength attacks. In Elkind, E., Veloso, M., Agmon, N., \& Taylor, M. E. (Eds.), Proc. of the 18th International Conference on Autonomous Agents and MultiAgent Systems, AAMAS '19, Canada, pp. 12701278. Int. Foundation for Autonomous Agents and Multiagent Systems.

Baroni, P., Romano, M., Toni, F., Aurisicchio, M., \& Bertanza, G. (2015). Automatic evaluation of design alternatives with quantitative argumentation. Argument \& Computation, 6(1), 24-49.

Barth, E. M., \& Krabbe, E. C. (2010). From axiom to dialogue: A philosophical study of logics and argumentation. Walter de Gruyter. 
Belinkov, Y., \& Glass, J. (2019). Analysis methods in neural language processing: A survey. Transactions of the Association for Computational Linguistics, 7, 49-72.

Bench-Capon, T. (2003). Persuasion in practical argument using value-based argumentation frameworks. Logic and Computation, 13(3), 429-448.

Bench-Capon, T. J. M. (2002). Value-based argumentation frameworks. In 9th International Workshop on Non-Monotonic Reasoning (NMR 2002), April 19-21, Toulouse, France, Proceedings, pp. 443-454.

Bistarelli, S., \& Taticchi, C. (2019). Power index-based semantics for ranking arguments in abstract argumentation frameworks. Intelligenza Artificiale, 13(2), 137-154.

Boella, G., Gabbay, D. M., van der Torre, L., \& Villata, S. (2010). Support in abstract argumentation. In Proceedings of the Third International Conference on Computational Models of Argument (COMMA'10), pp. 40-51. Frontiers in Artificial Intelligence and Applications, IOS Press.

Bonzon, E., Delobelle, J., Konieczny, S., \& Maudet, N. (2016). A comparative study of rankingbased semantics for abstract argumentation. In Thirtieth AAAI Conference on Artificial Intelligence.

Bonzon, E., Maudet, N., \& Moretti, S. (2014). Coalitional games for abstract argumentation.. In COMMA, pp. 161-172.

Budán, M. C. D., Gómez Lucero, M., Viglizzo, I., \& Simari, G. R. (2015). A labeled argumentation framework. Applied Logic, 13(4), 534-553.

Budán, M. C. D., Viglizzo, I., \& Simari, G. R. (2014). A labeled abstract bipolar argumentation framework. In Advances in Artificial Intelligence, pp. 28-40. Springer.

Budán, M. C., Lucero, M. G., Chesñevar, C., \& Simari, G. R. (2015). Modeling time and valuation in structured argumentation frameworks. Information Sciences, 290, 22-44.

Budán, M. C., Simari, G. I., Viglizzo, I., \& Simari, G. R. (2017). An approach to characterize graded entailment of arguments through a label-based framework. International Journal of Approximate Reasoning, 82, 242-269.

Budán, M. C., Gómez Lucero, M. J., Chesñevar, C. I., \& Simari, G. R. (2015). Modeling time and valuation in structured argumentation frameworks. Inf. Sci., 290, 22-44.

Budán, M. C. D., Cobo, M. L., Martínez, D. I., \& Rotolo, A. (2018). Dealing with qualitative and quantitative features in legal domains. In Legal Knowledge and Information Systems - JURIX 2018: The Thirty-first Annual Conference, The Netherlands, pp. 176-180.

Budán, M. C. D., Simari, G. I., \& Simari, G. R. (2016). Using argument features to improve the argumentation process. In Proceedings of Computational Models of Argument, pp. 151-158.

Cayrol, C., \& Lagasquie-Schiex, M. C. (2005a). Graduality in argumentation. Journal of Artificial Intelligence Research (JAIR), 23, 245-297.

Cayrol, C., \& Lagasquie-Schiex, M. C. (2005b). On the acceptability of arguments in bipolar argumentation frameworks. In Proc. of ECSQARU, pp. 378-389. Springer.

Cayrol, C., \& Lagasquie-Schiex, M. C. (2009). Bipolar abstract argumentation systems. In Rahwan, I., \& Simari, G. R. (Eds.), Argumentation in Artificial Intelligence, pp. 65-84. Springer. 
Cayrol, C., \& Lagasquie-Schiex, M. C. (2013). Bipolarity in argumentation graphs: Towards a better understanding. International Journal of Approximate Reasoning, 54(7), 876-899.

Chesñevar, C. I., \& Maguitman, A. G. (2004). Arguenet: An argument-based recommender system for solving web search queries. In Intelligent Systems, Vol. 1, pp. 282-287. IEEE.

Chesñevar, C., Modgil, S., Rahwan, I., Reed, C., Simari, G., South, M., Vreeswijk, G., Willmott, S., et al. (2006). Towards an argument interchange format. The knowledge engineering review, 21(4), 293-316.

Cohen, A., Gottifredi, S., García, A. J., \& Simari, G. R. (2014a). A survey of different approaches to support in argumentation systems. Knowledge Engineering Review, 29, 513-550.

Cohen, A., Gottifredi, S., García, A. J., \& Simari, G. R. (2014b). A survey of different approaches to support in argumentation systems. The Knowledge Engineering Review, 29(5), 513.

Cohen, A., Parsons, S., Sklar, E. I., \& McBurney, P. (2018). A characterization of types of support between structured arguments and their relationship with support in abstract argumentation. Int. J. Approx. Reasoning, 94, 76-104.

Cruz-Reyes, L., Trejo, C. M., Irrarragorri, F. L., \& Santillán, C. G. G. (2014). A decision support system framework for public project portfolio selection with argumentation theory. In Recent Advances on Hybrid Approaches for Designing Intelligent Systems, pp. 467-479. Springer.

Domshlak, C., Hüllermeier, E., Kaci, S., \& Prade, H. (2011). Preferences in AI: an overview. Artif. Intell., 175(7-8), 1037-1052.

Doutre, S., \& Mailly, J. (2017). Comparison criteria for argumentation semantics. In Belardinelli, F., \& Argente, E. (Eds.), Multi-Agent Systems and Agreement Technologies - 15th European Conference, EUMAS 2017, and 5th International Conference, AT 2017, France, Revised Selected Papers, Vol. 10767 of Lecture Notes in Computer Science, pp. 219-234. Springer.

Dubois, D., \& Prade, H. (1982). A class of fuzzy measure based on triangular norms? a general framework for the combination of uncertain information. International Journal Of General Systems, 8(1), 43-61.

Dung, P. M. (1995). On the acceptability of arguments and its fundamental role in nonmonotonic reasoning and logic programming and $n$-person games. Artificial Intelligence, 77, 321-357.

Elvang-Gøransson, M., Krause, P., \& Fox, J. (1993). Dialectic resoning with inconsistent information. In Proceedings of the Ninth international conference on Uncertainty in artificial intelligence, pp. 114-121. Morgan Kaufmann Publishers Inc.

Fazzinga, B., Flesca, S., \& Parisi, F. (2015). On the complexity of probabilistic abstract argumentation frameworks. ACM Trans. Comput. Log., 16(3), 22.

Fox, J., Glasspool, D., Grecu, D., Modgil, S., South, M., \& Patkar, V. (2007). Argumentation-based inference and decision making-a medical perspective. IEEE intelligent systems, 22(6), 34-41.

Gabbay, D. (1993). Labelled deductive systems: a position paper. In Oikkonen, J., \& Vaananen, J. (Eds.), Proceedings of Logic Colloquium '90, Vol. 2 of Lecture Notes in Logic, pp. 66-88. Springer-Verlag.

Gabbay, D. (1996). Labelling Deductive Systems (vol.1). Oxford University Press (Volume 33 of Oxford Logic Guides). 
Gabbay, D., \& Rodrigues, O. (2012). A numerical approach to the merging of argumentation networks. In Computational Logic in Multi-Agent Systems, pp. 195-212. Springer.

García, A. J., \& Simari, G. R. (2014). Defeasible logic programming: Delp-servers, contextual queries, and explanations for answers. Arg. and Comp., 5(1), 63-88.

Gorogiannis, N., \& Hunter, A. (2011). Instantiating abstract argumentation with classical logic arguments: Postulates and properties. Artificial Intelligence, 175(9-10), 1479-1497.

Hunter, A., Polberg, S., \& Thimm, M. (2020). Epistemic graphs for representing and reasoning with positive and negative influences of arguments. Artificial Intelligence, 281, 103236.

Hunter, A., \& Thimm, M. (2017). Probabilistic reasoning with abstract argumentation frameworks. Journal of Artificial Intelligence Research, 59, 565-611.

Janssen, J., De Cock, M., \& Vermeir, D. (2008). Fuzzy argumentation frameworks. In Information Processing and Management of Uncertainty in Knowledge-based Systems, pp. 513-520.

Kaci, S. (2011). Working with Preferences: Less Is More. Cognitive Technologies. Springer.

Kaci, S., \& van der Torre, L. (2008). Preference-based argumentation: Arguments supporting multiple values. International Journal of Approximate Reasoning, 48(3), 730-751.

Kraus, S. (1997). Negotiation and cooperation in multi-agent environments. Artif. Intell., 94(1), 79-97.

Leite, J., \& Martins, J. (2011). Social abstract argumentation. In Walsh, T. (Ed.), IJCAI 2011, Proceedings of the 22nd International Joint Conference on Artificial Intelligence, pp. 22872292, Barcelona, Catalonia, Spain. IJCAI/AAAI.

Lippi, M., \& Torroni, P. (2016). Argumentation mining: State of the art and emerging trends. ACM Trans. Internet Techn., 16(2), 10:1-10:25.

Lukasiewicz, T., \& Straccia, U. (2008). Managing uncertainty and vagueness in description logics for the semantic web. Web Semantics: Science, Services and Agents on the World Wide Web, 6(4), 291-308.

Martínez, D. C., \& Rotstein, N. D. (2008). Towards an abstract characterization of the subargument relation. In XIV Congreso Argentino de Ciencias de la Computación.

Matt, P.-A., \& Toni, F. (2008). A game-theoretic measure of argument strength for abstract argumentation. In European Workshop on Logics in Artificial Intelligence, pp. 285-297. Springer.

Nouioua, F., \& Risch, V. (2010). Bipolar argumentation frameworks with specialized supports. In 22nd International Conference on Tools with Artificial Intelligence, Vol. 1, pp. 215-218. IEEE.

Nunes, E., Shakarian, P., \& Simari, G. I. (2018). At-risk system identification via analysis of discussions on the darkweb. In 2018 APWG Symposium on Electronic Crime Research (eCrime), pp. 1-12. IEEE.

Palmirani, M. (2018). Dealing with qualitative and quantitative features in legal domains. In Legal Knowledge and Information Systems: JURIX 2018: The Thirty-first Annual Conference, Vol. 313, p. 176. IOS Press. 
Polberg, S., \& Hunter, A. (2018). Empirical evaluation of abstract argumentation: Supporting the need for bipolar and probabilistic approaches. International Journal of Approximate Reasoning, 93, 487-543.

Pollock, J. L. (2010). Defeasible reasoning and degrees of justification. Arg. and Comp., 1(1), 7-22.

Poole, D. L., Mackworth, A. K., \& Goebel, R. (1998). Computational intelligence: a logical approach, Vol. 79. Oxford University Press New York.

Potyka, N. (2018). Continuous dynamical systems for weighted bipolar argumentation. In Sixteenth International Conference on Principles of Knowledge Representation and Reasoning, pp. $148-157$.

Prakken, H., \& Sartor, G. (1997). Argument-based extended logic programming with defeasible priorities. Applied Non-classical Logics, 7, 25-75.

Prakken, H. (2014). On support relations in abstract argumentation as abstractions of inferential relations. In Proceedings of the 21st European Conference on Artificial Intelligence (ECAI 2014), Vol. 263, pp. 735-740. IOS Press.

Prakken, H., \& Sartor, G. (Eds.). (1997). Logical models of legal argumentation. Springer, Dordrecht, The Netherlands.

Rago, A., Čyras, K., \& Toni, F. (2016a). Adapting the df-quad algorithm to bipolar argumentation..

Rago, A., Toni, F., Aurisicchio, M., Baroni, P., et al. (2016b). Discontinuity-free decision support with quantitative argumentation debates.. $K R, 16,63-73$.

Rahwan, I., \& Simari, G. R. (2009). Argumentation in Artificial Intelligence. Springer Verlag.

Rossi, F. (2012). Preference reasoning and aggregation: Between AI and social choice. In Anthony, P., Ishizuka, M., \& Lukose, D. (Eds.), Proc PRICAI 2012: Trends in Artificial Intelligence - 12th Pacific Rim International Conference on Artificial Intelligence, Vol. 7458 of Lecture Notes in Computer Science, p. 2. Springer.

Rossi, F., Brent Venable, K., \& Walsh, T. (2011). A Short Introduction to Preferences: Between Artificial Intelligence and Social Choice. Synthesis Lectures on Artificial Intelligence and Machine Learning. Morgan \& Claypool Publishers.

Schweizer, B., \& Sklar, A. (1961). Associative functions and statistical triangle inequalities. Publ. Math Debrecem, 8, 169-186.

Schweizer, B., \& Sklar, A. (1963). Associative functions and abstract semigroups. Publ. Math Debrecem, $69-81$.

Shakarian, P., Simari, G. I., Moores, G., Paulo, D., Parsons, S., Falappa, M. A., \& Aleali, A. (2016). Belief revision in structured probabilistic argumentation: Model and application to cyber security. Ann Math Artif Intell, 78-259.

Simari, G. R., \& Loui, R. P. (1992). A mathematical treatment of defeasible reasoning and its implementation. Artificial intelligence, 53(2), 125-157.

Tamani, N., \& Croitoru, M. (2014). Fuzzy argumentation system for decision support. In Information Processing and Management of Uncertainty in Knowledge-Based Systems, pp. 77-86. Springer.

Toulmin, S. E. (2003). The Uses of Argument. Cambridge university press. 
Vreeswijk, G. A. W. (1997). Abstract argumentation systems. Artificial intelligence, 90(1), 225279.

Yager, R. (1983). Entropy and specificity in a mathematical theory of evidence. Gen. Syst., 9(4), 249-260.

Zimmermann, H. J. (2001). Fuzzy set theory—and its applications. Kluwer Academic Publishers, Boston. 